\title{
Pleistocene leopards in the Iberian Peninsula: New evidence from palaeontological and archaeological contexts in the Mediterranean region
}

\author{
Alfred Sanchis a, *, Carmen Tormo a, Víctor Sauqué ${ }^{\mathrm{b}}$, Vicent Sanchis ${ }^{\mathrm{c}}$, Rebeca Díaz ${ }^{\mathrm{c}}$, \\ Agustí Ribera ${ }^{\mathrm{d}}$, Valentín Villaverde ${ }^{\mathrm{e}}$ \\ a Museu de Prehistòria de València, Servei d'Investigació Prehistòrica, Diputació de València, València, Spain \\ ${ }^{\mathrm{b}}$ Grupo Aragosaurus-IUCA, Departamento de Ciencias de la Tierra, Facultad de Ciencias, Universidad de Zaragoza, Zaragoza, Spain \\ c Club d'Espeleologia l'Avern, Ontinyent, Spain \\ ${ }^{\mathrm{d}}$ Museu Arqueologic d'Ontinyent $i$ la Vall d'Albaida (MAOVA), Ontinyent, Spain \\ e Departament de Prehistòria i Arqueologia, Universitat de València, Valencia, Spain
}

\section{A R T I C L E I N F O}

\section{Article history:}

Received 7 April 2015

Received in revised form

7 July 2015

Accepted 11 July 2015

Available online $\mathrm{xxx}$

\section{Keywords:}

Panthera pardus

Late Pleistocene

Taxonomy

Distribution

Taphonomy

Iberian Peninsula

\begin{abstract}
A B S T R A C T
This study analyses the fossil record of leopards in the Iberian Peninsula. According to the systematic and morphometric features of new remains, identified mainly in Late Pleistocene palaeontological and archaeological sites of the Mediterranean region, they can be attributed to Panthera pardus Linnaeus 1758. The findings include the most complete leopard skeleton from the Iberian Peninsula and one of the most complete in Europe, found in a chasm (Avenc de Joan Guitón) south of Valencia. The new citations and published data are used to establish the leopard's distribution in the Iberian Peninsula, showing its maximum development during the Late Pleistocene. Some references suggest that the species survived for longer here (Lateglacial-Early Holocene) than in other parts of Europe. Finally, the contexts of appearance and origin of leopard remains are described and the processes of interaction with prehistoric human groups are assessed.
\end{abstract}

๑) 2015 Elsevier Ltd. All rights reserved.

\section{Introduction}

The leopard (Panthera pardus Linnaeus 1758) is a medium-sized member of the Felidae family with solitary, territorial habits. The males are usually heavier and have larger body dimensions than females. Their ubiquitous and eurythermal nature and their opportunistic behaviour allow them to adapt well to different biotopes. Nowadays, different subspecies are distributed throughout different areas of Africa and Asia (Turner and Antón, 1997; Bertram, 1999; Hayward et al., 2006; Macdonald et al., 2010; Stein and Hayssen, 2013).

The species is now extinct in Europe, but during the Pleistocene it was widely distributed (Sommer and Benecke, 2006), with occurrences throughout much of this continent (Bonifay, 1971;

\footnotetext{
* Corresponding author. Museu de Prehistòria de València, Servei d'Investigació Prehistòrica, Diputació de València, Corona 36, 46003 València, Spain.

E-mail address: alfred.sanchis@dival.es (A. Sanchis).
}

Kotsakis and Palombo, 1979; Spassov and Raychev, 1997; Fischer, 2000; Cardoso and Regala, 2006; Baryshnikov, 2011; Marciszak et al., 2011; Testu et al., 2011; Altuna and Mariezkurrena, 2013; Diedrich, 2013; Sauqué and Cuenca-Bescós, 2013; Sauqué et al., 2014a; Ghezzo and Rook, 2015). The earliest appearance of P. pardus in Europe could be the Early Pleistocene remains found at Le Vallonnet (Moullé et al., 2006), although most leopard identifications in ancient contexts have been questioned and the remains reassigned to Puma pardoides (Hemmer, 2001; Argant, 2004; Hemmer et al., 2004; Madurell-Malapeira et al., 2010; Cherin et al., 2013). The presence of other felines during this phase, such as the puma, jaguar or cheetah, could explain the later appearance of the leopard (Testu, 2006). The first confirmed European evidence of the leopard corresponds to the Middle Pleistocene, with its maximum expansion towards the end of this phase and the beginning of the Late Pleistocene (Turner, 1995; Crégut-Bonnoure, 1996; Testu, 2006). The species' survival in Europe is uneven and varies geographically; in France the last appearances correspond to the Early Upper Palaeolithic, before the Last Glacial Maximum 
(LGM) (Bonifay, 1971; Crégut-Bonnoure, 1996), in Jaurens (Ballesio, 1980) and Isturitz (Altuna, 1972). In Italy, the last occurrences appear in the MIS 3 levels of Equi Cave (Ghezzo and Rook, 2015), in the Aurignacian levels of Fumane Cave (Cassoli and Tagliacozzo, 1991) and the LGM levels of Arene Candide (Cassoli and Tagliacozzo, 1994; Sommer and Benecke, 2006). In central Europe it has been considered that the last appearance of the leopard would date to the Lower Pleniglacial (Wolsan, 1993), but two records in Ettingen, Switzerland, and Teufelsbrücke, Germany, suggest that the species may have survived in the area during the Lateglacial (cited in Sauqué and Cuenca-Bescós, 2013). In the Balkans (Triagalnata) the species is recorded during the Lateglacial
(Spassov and Raychev, 1997; Sommer and Benecke, 2006). The last appearance of leopard on the continent is documented in Greece (Vraona) (Nagel, 1999) and in the Iberian Peninsula (see sites listed in Sauqué and Cuenca-Bescós, 2013; see also Table 13 in this study), with several records during the Lateglacial and Early Holocene. Some marginal areas of the continent could have acted as a refuge for the species during the harshest phases (O'Regan, 2008).

In the Iberian Peninsula, the leopard's presence during the Middle Pleistocene is limited and most appearances are from the Late Pleistocene onward (Sauqué and Cuenca-Bescós, 2013). They are normally assemblages consisting of few remains (isolated teeth, metapodials or phalanges) that are found in Middle and Upper

Table 1

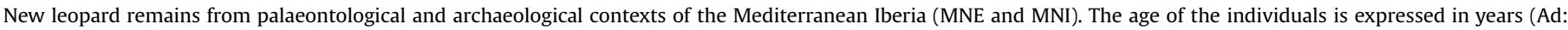
adult).

\begin{tabular}{|c|c|c|c|c|c|c|c|c|c|}
\hline Elements & AJG & Malladetes & REC I & REC II & Bolomor & C. Negra & Horadada & C. Borràs & Meravelles \\
\hline Cranium & 1 & & & & & & & & \\
\hline Maxilla & & & & 1 & & & & & \\
\hline Mandible & 1 & & & & & & & & \\
\hline $\mathrm{C} 1$ & & & 1 & & & & & & \\
\hline i1 & 1 & & & & & & & & \\
\hline $\mathrm{m} 1$ & & 1 & & & & & & & \\
\hline Scapula & 2 & & & & & & & & \\
\hline Humerus & 2 & & & 2 & & & & & \\
\hline Radius & 2 & & 1 & 2 & & & & & \\
\hline Ulna & 2 & & & 2 & & & & 1 & \\
\hline Scapholunate & 2 & & 1 & 1 & & & & & \\
\hline Pyramidal & 2 & & & & & & & & \\
\hline Pisiform & 2 & & 1 & 1 & & & & & \\
\hline Trapezium & 2 & & & & & & & & \\
\hline Trapezoid & 2 & & & & & & & & \\
\hline Capitate & 2 & & & & & & & & \\
\hline Hamate & 2 & & & & & & & & \\
\hline Mc I & 2 & & & 1 & & & & & \\
\hline Mc II & 2 & & 2 & 1 & & & & & \\
\hline Mc III & 2 & & 1 & & & & & & \\
\hline Mc IV & 2 & & 2 & 1 & & 1 & & & 1 \\
\hline Mc V & 2 & & & & 2 & & & & \\
\hline Hyoid & 1 & & & & & & & & \\
\hline Sternum & 8 & & & & & & & & \\
\hline Cervical V. & 7 & & & & & & & & \\
\hline Thoracic V. & 13 & & & & & & & & \\
\hline Lumbar V. & 7 & & & 1 & & & & & \\
\hline Sacrum & 1 & & & & & & & & \\
\hline Caudal V. & 22 & & 8 & 1 & & & & & \\
\hline Ind. V. & & & 1 & & & & & & \\
\hline Ribs & 25 & & 2 & & & & & & \\
\hline Pelvis & 1 & 1 & & & & & & & \\
\hline Femur & 2 & & & & & & & & \\
\hline Tibia & 2 & & 1 & 1 & & & & & \\
\hline Fibula & 1 & & & & & & & & \\
\hline Patella & 2 & & 1 & & & & & & \\
\hline Calcaneus & 2 & & & 2 & & 1 & & & \\
\hline Talus & 1 & & 1 & 1 & & & & & \\
\hline Cuboid & 2 & & & 1 & & & & & \\
\hline Navicular & 2 & & & 1 & & & & & \\
\hline Cuneiform I & 2 & & & & & & & & \\
\hline Cuneiform II & 1 & & & & & & & & \\
\hline Cuneiform III & 2 & & & 1 & & & & & \\
\hline Mt I & 2 & & & & & & & & \\
\hline Mt II & 2 & & & 1 & & & & & \\
\hline Mt III & 1 & & 1 & & 1 & & & & \\
\hline Mt IV & 2 & & 1 & 1 & 1 & & & & \\
\hline Mt V & 2 & & 1 & 1 & & & & & \\
\hline 1st phalanx & 17 & 2 & 8 & 5 & & & 1 & & \\
\hline 2nd phalanx & 16 & & 5 & 5 & & 1 & & & \\
\hline 3rd phalanx & 15 & & 1 & 2 & & & & & \\
\hline Sesamoid & 25 & & & & & & & & \\
\hline Metapodial & & & & 2 & & & & & \\
\hline Total MNE & 221 & 4 & 40 & 38 & 4 & 3 & 1 & 1 & \\
\hline Total MNI & 1 & 2 & 2 & 1 & 2 & 1 & 1 & 1 & 1 \\
\hline Age & 1.5 to 2 & $<1 / \mathrm{Ad}$ & $\mathrm{Ad} / 7$ to 10 & 3 to 4 & $\mathrm{Ad} / \mathrm{Ad}$ & Ad & Ad & Ad & Ad \\
\hline
\end{tabular}


Palaeolithic archaeological sites or palaeontological contexts, while partial or complete skeletons are rare (Arribas, 1997; Cardoso and Regala, 2006).

Various taphonomic studies have shown the leopard's ability to accumulate and modify bone remains in archaeological sites (de Ruiter and Berger, 2000; Domínguez-Rodrigo and Pickering, 2010; Sauqué et al., 2014b; Gidna et al., 2015) as a consequence of competition with hominins and other carnivores for access to prey and occupation of caves (Brugal and Fosse, 2004). The leopard is a solitary, territorial hunter (Bertram, 1999; Hayward et al., 2006) that acts on all types of prey, although it mainly targets those within a weight range of 20-80 kg (Mills and Harvey, 2001). Despite not being a selective hunter (Hart et al., 1996; Hayward et al., 2006), it prefers prey with an optimum size of $23 \mathrm{~kg}$ (Hayward et al., 2006).

As regards the taxonomy of the European Pleistocene leopard, the theory put forward by Diedrich (2013) is that there are four subspecies, the most recent being $P$. pardus spelaea Bächler 1936, also known as the "Ice Age Leopard", a subspecies from the Late Pleistocene with its own skeletal morphology and differences with respect to the present-day leopard.

In contrast to this, Ghezzo and Rook (2015) recently suggested that all the European leopards belong to a single species, $P$. pardus, although there is no genetic analysis yet. This theory is based on morphological differences in the Equi population that they link to intraspecific variability.

This paper presents new leopard remains from Pleistocene palaeontological and archaeological contexts in the Mediterranean region of the Iberian Peninsula. Part of this study will describe a leopard skeleton recovered in Avenc de Joan Guitón (southern
Valencia), the most complete example found in this geographical area, which provides very valuable information and enables us to compare its morphometric characteristics with other findings. A review and study is also made of remains from ancient excavations in the Valencia area. Based on the morphometric characteristics of several of the remains studied here, they can be attributed to $P$. pardus, although they show many similarities with the subspecies $P$. pardus spelaea.

The purpose of this study is also to review and compile the information, so all citations of leopards in the Iberian Peninsula corresponding to archaeological and palaeontological contexts are recorded in order to analyse the chronology of the species' appearance, expansion and disappearance in the area. Attention is also given to the origin of the remains (natural or anthropogenic) and the characteristics of the contexts of appearance in order to describe the possible processes of interaction between leopards and prehistoric human groups.

\section{Methods and materials}

\subsection{Methodology}

The reference collections of the Museu de Prehistòria de València (MPV) and Universidad de Zaragoza were used for the taxonomic identification of the leopard remains, as was the bone collection of archeozoo.org The following fossil collections were consulted: Museu de Ciències Naturals de Barcelona, Museu Geològic del Seminari de Barcelona, Museo de Huesca, Museo de Ciencias Naturales de la Universidad de Zaragoza, Museo Nacional de Ciencias Naturales in Madrid, Instituto Alavés de la Naturaleza

Table 2

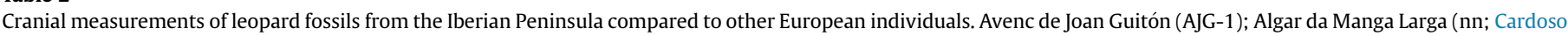

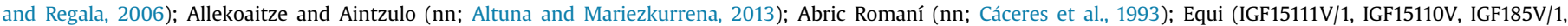

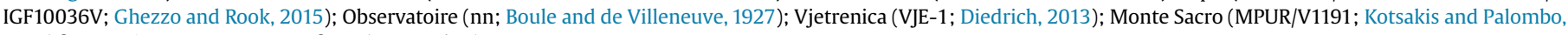
1979) [Approximate measurement]. nn (no number).

\begin{tabular}{|c|c|c|c|c|c|c|c|c|c|c|}
\hline Crania & & AJG & AML & ALL & AIN & ARO & EQC & OBS & VJE & MSA \\
\hline 1 & Total L & 195.2 & 183 & 221.7 & [210.5] & & 191-196-255-239 & 223 & 214 & 250.5 \\
\hline 2 & Condylobasal L & 184.8 & 165 & 204.6 & [195] & & & 209 & & \\
\hline 3 & Basal L & 166.5 & 152.6 & 192.6 & 184.5 & & $170-175-210-195.8$ & & & \\
\hline 4 & Basicranial axis & 541 & & 64.1 & & & & & & \\
\hline 5 & Basifacial axis & 110.9 & & 130.2 & & & & & & \\
\hline 6 & Neurocranium L & 122 & 131.1 & 138 & 127 & & & & & \\
\hline 7 & Upper neurocranium L & 106.7 & [105.7] & & & & & & & \\
\hline 8 & Viscerocranium L & 100 & [101.5] & & & 97 & & & & \\
\hline 9 & Facial L & 67.2 & & 94.5 & [91] & & & & & \\
\hline 10 & Lateral L snout & 65.3 & 57.2 & & & & & & & \\
\hline 11 & Median palatal L & 90.9 & & & & & & 83 & & \\
\hline $11 \mathrm{a}$ & Palatal L & 94.1 & 76.2 & 100.5 & [98.5] & & & & & \\
\hline 12 & Cheektooth row $\mathrm{L}$ & 48.5 & 45.7 & 53.4 & & & & & & \\
\hline 13 & Premolar row L & 46.6 & 43.4 & 48.9 & 52.5 & 49.9 & & & & \\
\hline 15 & P4 alveolus L & 23.1 & 21.3 & & & & & & & \\
\hline 16 & Greatest D auditory bulla & 33 & 24 & 35.2 & 30 & & & & & \\
\hline 17 & Least D auditory bulla & 22.4 & 18.8 & 25.3 & 20.5 & & & & & \\
\hline 18 & Greatest mastoid B & 85.7 & 79.2 & 93 & {$[86]$} & & & 93 & & \\
\hline 19 & Greatest B occip. condyles & {$[40]$} & [39] & 42.9 & [41] & & $39.6-41.6-49-48$ & 43 & 44.7 & 48 \\
\hline 20 & Greatest B fora. magnum & 22.7 & 21.7 & 23.7 & 19.2 & & & & & \\
\hline 21 & $\mathrm{H}$ foramen magnum & 18.7 & 17.2 & 15.8 & 15.9 & & & & & \\
\hline 22 & Greatest neurocranium B & 72.4 & 69.1 & & & & $69.8-78.4-80-75.9$ & & & \\
\hline 23 & Zygomatic B & 130 & $>129.4$ & & & & & 150 & & \\
\hline 24 & Frontal B & 70.6 & $>72.2$ & & & & & 68 & & \\
\hline 25 & Least B between the orbits & 43.3 & 43.1 & & & & & 44 & & \\
\hline 26 & Greatest palatal B & 77.5 & 75.9 & & & & $86.7-91.2-112-89.6$ & 91 & & \\
\hline 27 & B canine alveoli & 52.8 & {$[53]$} & 60 & 56.5 & & & 60 & & \\
\hline 28 & Least B aboral supraorb. p. & 44.2 & 45.2 & 43.4 & 44.5 & & & 43 & & \\
\hline 29 & Facial $\mathrm{B}$ between infraorb. $\mathrm{f}$. & 55.7 & 54.4 & 51.7 & 61 & & & & & \\
\hline 30 & Greatest inner L orbit & 35.4 & $>33.1$ & & & 36 & & & & \\
\hline 31 & Greatest inner $\mathrm{H}$ orbit & 41.6 & 40.4 & & & & & & & \\
\hline 32 & H occipital triangle & [52.9] & 52.1 & 58.8 & & & & & & \\
\hline
\end{tabular}


Table 3

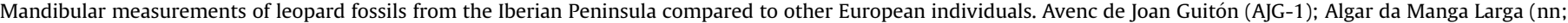

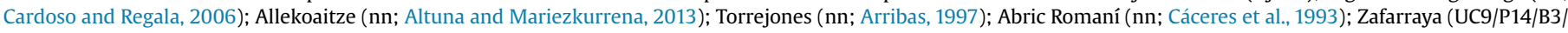

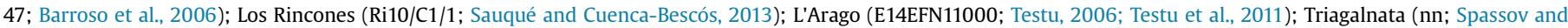
Raychev, 1997); Karaïn E (17H26110, 18H26111; Testu, 2006). [Approximate measurement]. nn (no number).

\begin{tabular}{|c|c|c|c|c|c|c|c|c|c|c|c|}
\hline \multicolumn{2}{|c|}{ Mandible } & \multirow{2}{*}{$\frac{\mathrm{AJG}}{135.1}$} & \multirow{2}{*}{$\frac{\text { AML }}{123.7}$} & \multirow{2}{*}{$\frac{\text { ALL }}{150.2}$} & \multirow[t]{2}{*}{ TOR } & \multirow{2}{*}{$\frac{\text { ARO }}{141.5}$} & \multirow{2}{*}{$\frac{B Z}{151.5}$} & \multirow{2}{*}{$\frac{\mathrm{LR}}{132}$} & \multirow{2}{*}{$\frac{\text { ARA }}{137.7}$} & \multirow{2}{*}{$\frac{\text { TRI }}{134}$} & \multirow[t]{2}{*}{ KAR } \\
\hline 1 & Total L & & & & & & & & & & \\
\hline 2 & L ident. Condyle/ang proc-infradent & 127.7 & 120.2 & 143.3 & & & & 122 & & & \\
\hline 3 & L cond. Proc. aboral border c1 alveolus & [111.8] & 107.1 & 129.2 & & 123.7 & & & & & \\
\hline 4 & L ident. Cond./ang. Proc.-abo. Bor. c1 alv. & {$[108.4]$} & 102.4 & 125.5 & & 118.1 & & & & & \\
\hline 5 & L cheektooh row $\mathrm{p} 3-\mathrm{m} 1$ alveoli & 45.7 & 44.3 & 51.1 & 44.7 & 49.2 & 53.8 & 49 & 43.7 & 49 & $50.5-50.3$ \\
\hline 7 & $\mathrm{~L} \mathrm{~m} 1$ alveolus & & 17.5 & & & & & 18.8 & & & $19.8-19$ \\
\hline 8 & $\mathrm{H}$ vertical ramus & 61.3 & 58.7 & & & & & 52.5 & 63.5 & & \\
\hline 9 & $\mathrm{H}$ mandible behind $\mathrm{m} 1$ & 29.5 & 27.7 & 30 & & & & 27.2 & 28.2 & 28 & 31.2 \\
\hline \multirow[t]{4}{*}{10} & $\mathrm{H}$ mandible in front $\mathrm{p} 3$ & 27 & 28.9 & 28.2 & & & 33 & & 27.4 & 26.5 & 31.3 \\
\hline & L diasteme $c 1-p 3$ & 15.3 & 11.4 & & & & 14.7 & & 14.2 & 10.1 & 15.9 \\
\hline & L p3-m1 & {$[46.5]$} & 44.3 & & & & & 32 & & 49 & $49.3-49.5$ \\
\hline & L p3-p4 & [29] & 27.3 & & & & & & 27.4 & & $31.7-31.2$ \\
\hline
\end{tabular}

(IAN) in Vitoria and North Carolina Museum of Natural Sciences and Smithsonian National Museum of Natural History in the United States. Various articles were also used: Schmid (1940), Testu (2006), Sims (2005, 2012), Christiansen (2008), Diedrich (2013), Sauqué and Cuenca-Bescós (2013), Indu et al. (2013), Podhade et al. (2013, 2014a and b), Sauqué et al. (2014a) and Ghezzo and Rook (2015). For the description of the cranial and mandibular material from Avenc de Joan Guitón (AJG), other well-preserved Late Pleistocene leopard skulls from the Iberian Peninsula were used as comparative paratypes: Algar da Manga Larga (Cardoso and Regala, 2006), Allekoaitze and Aintzulo (Corral, 2012; Altuna and Mariezkurrena, 2013). Where the remains of both sides are present, the best preserved element is used in the morphological description, provided that no differences are observed. The fossil remains presented in this paper are deposited in the MPV.

The new specimens were quantified according to the minimum number of elements (MNE) and the minimum number of individuals (MNI) (Lyman, 2008). The age at death was estimated by the state of tooth wear (Stander, 1997) and the degree of joint fusion of the bones (Morris, 1972). The sex was determined according to the cranial features described by Ghezzo and Rook (2015), although the cranial and postcranial dimensions of the remains were also taken into account as a guideline (Diedrich, 2013). Elements with trauma or fractures were described according to Bartosiewicz (2013).

Measurements are expressed in millimetres and were taken using digital callipers (TESA CAL-IP67, Swiss) according to the method proposed by von den Driesch and von (1976). All metric information is shown in Tables $2-12$, where the dimensions of the new remains are presented alongside those of published materials, mainly from the Iberian Peninsula. For comparative purposes, we also present the skull and dentition measurements of present-day leopards, pumas and snow leopards, recorded by Testu (2006) and Cardoso and Regala (2006) (see Supplementary material).

\section{Table 4}

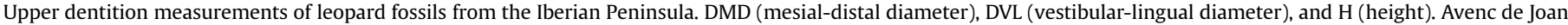

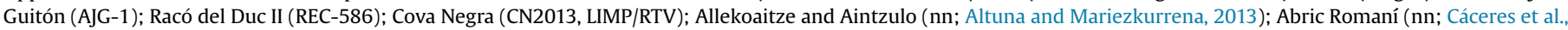

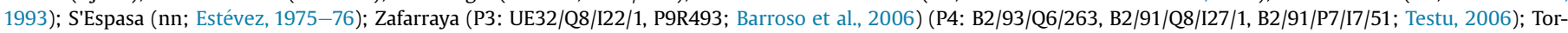

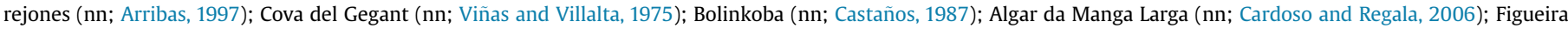
Brava, Gruta do Escoural and Lorga da Dine (nn; Cardoso, 1993, 1996). [Approximate measurement]. nn (no number).

\begin{tabular}{|c|c|c|c|c|c|c|c|c|c|c|c|c|c|c|c|c|}
\hline \multicolumn{2}{|c|}{ Upper dentition } & \multirow[t]{2}{*}{ AJG } & \multirow[t]{2}{*}{ REC II } & \multirow[t]{2}{*}{$\mathrm{CN} 3$} & \multirow[t]{2}{*}{ ALL } & \multirow[t]{2}{*}{ AIN } & \multirow[t]{2}{*}{ ARO } & \multirow[t]{2}{*}{ ESP } & \multirow[t]{2}{*}{$\mathrm{BZ}$} & \multirow[t]{2}{*}{ TOR } & \multirow[t]{2}{*}{ GEG } & \multirow[t]{2}{*}{ BOK } & \multirow[t]{2}{*}{ AML } & \multirow[t]{2}{*}{ FB } & \multirow[t]{2}{*}{ ESC } & \multirow[t]{2}{*}{ LDD } \\
\hline I1 & DMD & & & & & & & & & & & & & & & \\
\hline & DVL & $3.7-3.2$ & & & & & & & & & & & & & & \\
\hline \multirow[t]{2}{*}{$\mathrm{I} 2$} & DMD & & $5.7-5.7$ & & & & & & & & & & & & & \\
\hline & DVL & $3.5-3.8$ & $3.9-4.1$ & & & & & & & & & & & & & \\
\hline \multirow[t]{2}{*}{ I3 } & DMD & & $8.7-9$ & & & & & & & & & & & & & \\
\hline & DVL & $4.4-4.5$ & $7.6-7.7$ & & & & & & & & & & & & & \\
\hline \multirow[t]{2}{*}{$\mathrm{C} 1$} & DMD & $14-14.1$ & & & & & & 12.5 & & & & $14.1-12.1-14.3-13.5$ & 12.3 & 12.9 & 14.7 & 14.3 \\
\hline & DVL & {$[12.7]-[12.7]$} & & & & & & & & & & $11.5-11-9.3-10.6$ & 9.8 & 10.5 & $10.2-12.2$ & 10.3 \\
\hline \multirow[t]{2}{*}{ P2 } & DMD & 4.4 & & & & & & & & & & & & & & \\
\hline & DVL & 3.1 & & & & & & & & & & & & & & \\
\hline \multirow[t]{4}{*}{ P3 } & DMD & $16.4-16.2$ & & & 18.7 & 19.2 & 18.7 & 19.4 & $17.3-20.3$ & & 17.5 & 19.1 & 15 & 16.2 & & \\
\hline & DVL & & & & 9.3 & 9.3 & 9.1 & 9.4 & $8.7-9.1$ & & 8 & 8.3 & 7.8 & 8 & & \\
\hline & DMD proto & & & & & & & & $12.9-14$ & & & & & & & \\
\hline & DMD talon & & & & & & & & $5.8-7.3$ & & & & & & & \\
\hline \multirow[t]{11}{*}{ P4 } & DMD ext & $23.2-23.3$ & & 26.5 & & 25.7 & 24.7 & {$[24.8]$} & $24.4-24.5-26.5$ & 22.6 & 26 & & 23.8 & 25.1 & $25.1-28.6$ & \\
\hline & DMD mid & & & 25.1 & & & 24.4 & & $23.1-24.2-25.5$ & & & & & & & \\
\hline & DMD int & & & 25.8 & & & 25.6 & & $24.1-24.2-26.4$ & & & & 23.4 & 25.1 & $26-27.9$ & \\
\hline & DVL ant & & & 13.3 & 13.9 & 13.3 & 12.9 & 13.5 & $12.1-12.9-13.7$ & 12 & & & 12 & 13 & $12.5-15$ & \\
\hline & DVL mid & & & 9.5 & & & & & & & & & & & & \\
\hline & DVL post & & & 8.2 & & & 8.3 & & 7.9-7.7-9.4 & & & & & & & \\
\hline & DMD para & $9.8-9.7$ & & 10.1 & & & 10.1 & & $11.2-9.9-11.3$ & & & & & & & \\
\hline & DMD meta & $8.8-8.7$ & & 11.1 & & & 10.4 & & $10.5-10.9-11.6$ & & & & 9.3 & 10.1 & $10.4-12.1$ & \\
\hline & DMD p + m & $17.9-18.2$ & & 20 & & & 19.6 & & 20-19.4-20.7 & & & & & & & \\
\hline & H para & 13.1 & & 13 & 13.2 & 12.5 & 13.3 & & $12.9-13.1-14.6$ & & & & & & & \\
\hline & H proto & & & 5.5 & & & 5.6 & & $4.3-3.8-4.7$ & & & & & & & \\
\hline
\end{tabular}




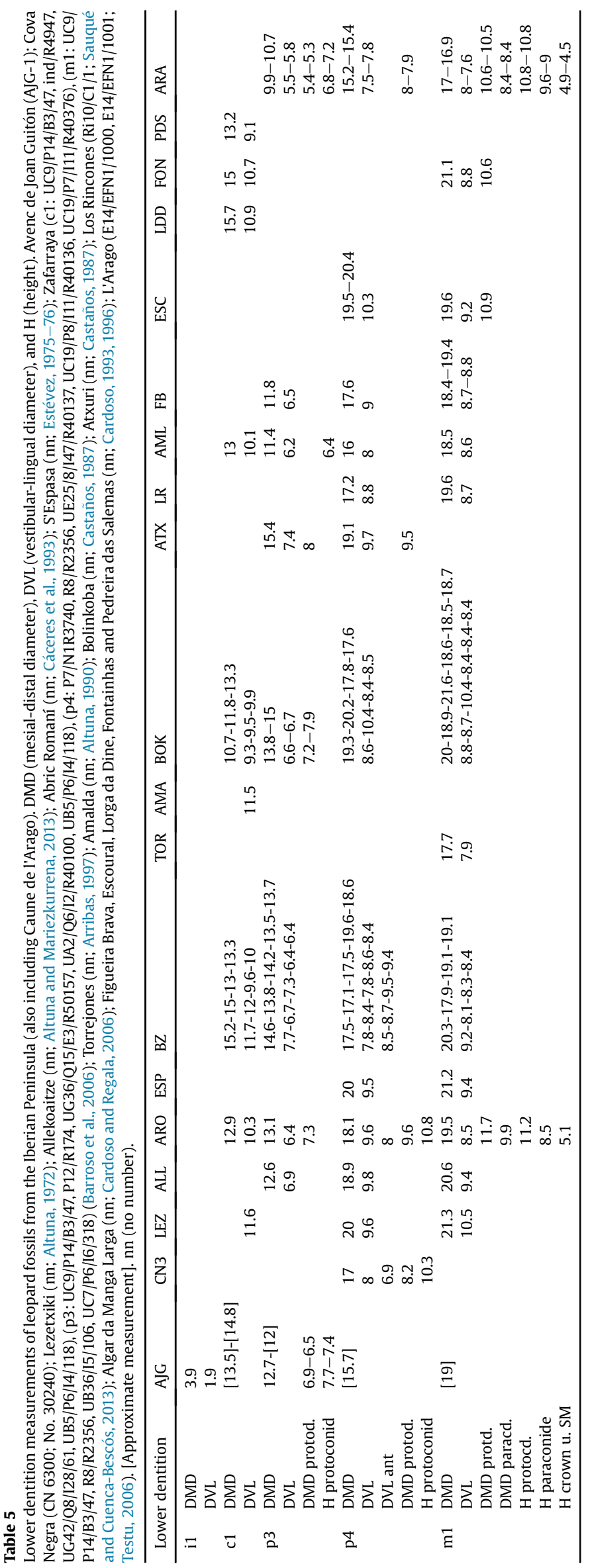

All citations of Iberian Peninsula leopards are shown in Fig. 14 with further details in Table 13, showing the altitude of the site, context of appearance, chronology of the finding, number of remains/individuals/presence, taxonomic attribution, level, bibliographical references and an identification number for each site.

\subsection{Sites and remains}

\subsubsection{Avenc de Joan Guitón}

The chasm is located in the vicinity of Fontanars dels Alforins, in the south of the province of Valencia (Spain), in the east of the Iberian Peninsula (Fig. 1A). It is situated at approximately 770 masl in an area covered by typical Mediterranean vegetation of pine trees and holm oaks known as Racó del Colmenar (Fig. 1B), its former name, in the upper axis of an anticline formed by Cretaceous calcareous material on the SE face of the Serra Grossa mountain range. AJG lies on the crossover point of two faults; the chasm runs along the first of these faults in a N-S direction, while the second (NE-SW) affects its continuity to the south, cutting across the fracture perpendicularly (Fig. 1C).

This place has been frequented by many speleological groups and has been the subject of various descriptive and topographic studies. In 2013, two of the authors (VS and RD), from the Club d'Espeleologia l'Avern d'Ontinyent, found various bone remains in a new, unexplored area.

AJG is a very complex cave in terms of its development and morphology (Fig. 2A). It consists of various karst systems running $\mathrm{N}$ and $S$ that reach -211 and $-161 \mathrm{~m}$, respectively, with horizontal galleries that form superimposed chambers. The Leopard Gallery is very difficult to access, as there are a number of technical difficulties with vertical sections that require speleological knowledge. After passing various pits, horizontal sections, slopes, loose blocks and narrow passages, we reach a small vertical fracture about $30 \mathrm{~cm}$ wide covered with boulders. After removing the boulders, we reach a flat-ceilinged gallery that is $40 \mathrm{~m}$ long ending in a $6-\mathrm{m}$ vertical section, where the bone remains are located at a depth of about $150 \mathrm{~m}$ in an area far away from the current vertical mouth to the cave (Fig. 2B). It would have been impossible for the animal to reach this place by itself via the current entrance, so it can be assumed that the cave had other closer entrances in the past.

The photographs taken showed that the remains belonged to a feline of the Panthera genus, possibly a leopard. The MPV and the Museu Arqueològic d'Ontinyent i la Vall d'Albaida (MAOVA) therefore undertook an initiative in collaboration with the aforementioned speleologists. The material was recovered with the authorization of the Directorate General for Heritage of the Valencian Regional Government (2013/0697-V (SS.TT.).

The feline skeleton was found in the area described (Fig. 2C). The materials were photographed and stored by anatomical group and side. The process was recorded by photography and video. The remains belong to an individual leopard and no other specimens were found. The different elements were found in anatomical semiconnection, some bones were joined by concretion and they are barely covered by sedimentation; others, such as the cranium, were somewhat displaced and half-buried.

Once the remains had been recovered and adequately packaged, they were moved to the MPV for cleaning and consolidation. Controlled drying was performed using ethyl alcohol and acetone impregnations to avoid stress. Some of the remains were in a very delicate state and they need to be consolidated with $10 \%$ and $20 \%$ Paraloid B-72. A complete consolidation and restoration plan was therefore developed. After these measures had been taken, it was observed that the front of the individual was better preserved than the rear and the right side was better than the left. Many of the remains presented different levels of concretion; the analyses 
Table 6

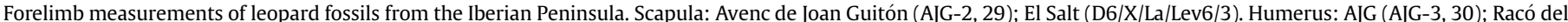

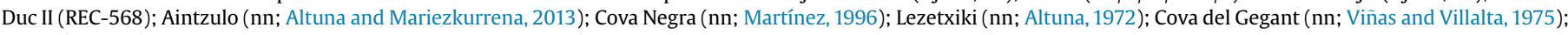

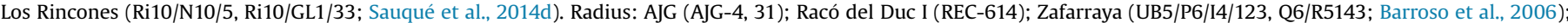

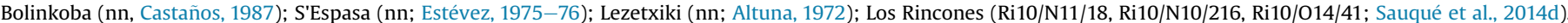

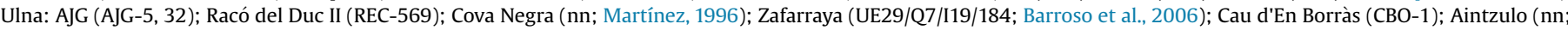

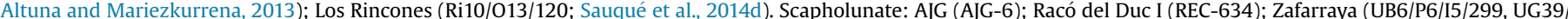

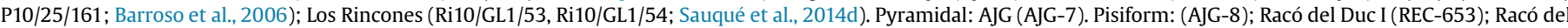

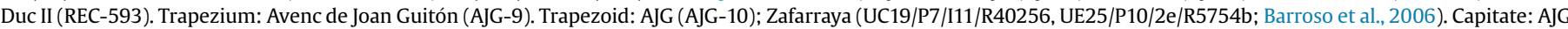
(AJG-11); Zafarraya (UE25/P19/D5/R40024; Barroso et al., 2006). Hamate: AJG (AJG-12). [Approximate measurement]. nn (no number).

\begin{tabular}{|c|c|c|c|c|c|c|c|}
\hline Scapula & AJG & ESA & & & & & \\
\hline BG & $24.6-24$ & 24.8 & & & & & \\
\hline GLP & $38.1-38$ & 42.4 & & & & & \\
\hline LG & $31.5-30.1$ & 34.8 & & & & & \\
\hline SLC & $34-32$ & 37 & & & & & \\
\hline Humerus & $\mathrm{AJG}$ & REC II & AIN & $\mathrm{CN} 2$ & LEZ & GEG & LR \\
\hline GL & $212-212$ & & 236.9 & & & & \\
\hline Bp ap & $53.4-54.2$ & & & & & & \\
\hline $\mathrm{Bpt}$ & $42.6-42$ & & & & & & \\
\hline SD & $19-18.4$ & & 18.4 & & & & \\
\hline Bd ap & 30.7-[31.8] & & & & & 34.6 & \\
\hline $\mathrm{Bd} \mathrm{t}$ & $53-[54.7]$ & 55.7 & 54.9 & 51.4 & 50 & 51 & $47.6-53.6$ \\
\hline Radius & $\mathrm{AJG}$ & REC I & $\mathrm{BZ}$ & BOK & ESP & LEZ & LR \\
\hline GL & 183.5 & 184.3 & & & & & \\
\hline Bp ap & $17.8-17.4$ & 16.5 & $17.2-18.4$ & & & & \\
\hline $\mathrm{Bpt}$ & $23.8-24.4$ & 24.8 & $23.6-27.1$ & 25.2 & 26.1 & $24-24.3$ & $22.2-23.2$ \\
\hline SD & 16.8 & 15.8 & & 17.8 & 17.1 & 18.8 & 15.8 \\
\hline Bd ap & 20.6-[22.1] & 21.3 & & & & & \\
\hline $\mathrm{Bd} \mathrm{t}$ & $34-[34.5]$ & 35.7 & & & 37.2 & 40.7 & 40.6 \\
\hline Ulna & $\mathrm{AJG}$ & REC II & $\mathrm{CN} 2$ & $\mathrm{BZ}$ & $\mathrm{CBO}$ & AIN & LR \\
\hline GL & 226 & & & & & & 241 \\
\hline Bp ap & $30.2-30$ & 42.1 & & 33.6 & & & \\
\hline $\mathrm{Bpt}$ & 21 & 22 & & 22.7 & & & \\
\hline LO & 40.7 & & & & & & \\
\hline SDO & 28.5 & & & & & 28.2 & \\
\hline DPA & $32.3-32.8$ & & & & {$[36.4]$} & 35.8 & \\
\hline $\mathrm{BPC}$ & 28.8 & & 27.8 & & [26.5] & & 30.81 \\
\hline Bd ap & 21.8 & & & & & & \\
\hline $\mathrm{Bd} \mathrm{t}$ & 10.5 & & & 12 & & & \\
\hline Scapholunate & AJG & REC I & $\mathrm{BZ}$ & LR & & & \\
\hline DAP & 20.3 & 21.9 & $25-25.6$ & & & & \\
\hline DT & 27.8 & 28.4 & $29.3-32$ & $29.9-28.8$ & & & \\
\hline $\mathrm{H}$ & 17.1 & 17.1 & $19.2-18.3$ & & & & \\
\hline Pyramidal & AJG & & & & & & \\
\hline DAP & 11.3 & & & & & & \\
\hline DT & 15.4 & & & & & & \\
\hline $\mathrm{H}$ & 7.3 & & & & & & \\
\hline Pisiform & AJG & REC I & REC II & & & & \\
\hline DAP & 23.4 & 24.7 & 27.3 & & & & \\
\hline DT & 14.8 & 14.6 & 15.7 & & & & \\
\hline $\mathrm{H}$ & 14.2 & 14.9 & 16.3 & & & & \\
\hline Trapezium & AJG & & & & & & \\
\hline DAP & 10 & & & & & & \\
\hline DT & 15.9 & & & & & & \\
\hline $\mathrm{H}$ & 9.5 & & & & & & \\
\hline Trapezoid & AJG & $\mathrm{BZ}$ & & & & & \\
\hline DAP & 12.6 & $15.6-13.8$ & & & & & \\
\hline DT & 14.4 & $17.4-18.2$ & & & & & \\
\hline $\mathrm{H}$ & 7 & $9.9-7.3$ & & & & & \\
\hline Capitate & AJG & $\mathrm{BZ}$ & & & & & \\
\hline DAP & 20.2 & 22.1 & & & & & \\
\hline DT & 13 & 10.1 & & & & & \\
\hline $\mathrm{H}$ & 13.5 & 14.8 & & & & & \\
\hline Hamate & AJG & & & & & & \\
\hline DAP & 17.7 & & & & & & \\
\hline DT & 12.5 & & & & & & \\
\hline $\mathrm{H}$ & 15.3 & & & & & & \\
\hline
\end{tabular}

carried out on two samples by Arte-Lab S. L. (XRD, FTIR, SEM-EDX) confirmed that it was calcium carbonate (aragonite) associated with contamination by clays, caused by external diagenetic processes. As a result of these processes the cranium and mandible were attached to each other, although for now their conservation is the priority and it has been decided that they should not be separated.
The skeleton is almost complete and the bones are fused (Table 1; Fig. 3). All the teeth were still in the maxilla and mandible, except for a lower incisor that was found on its own. Some bones are missing from the hindlimb. As regards the age at death of the individual, it has all its permanent dentition but it is not worn, so it could have been between 1.5 and 2 years old (Stander, 1997).

Signs of trauma and/or fractures were found on several of the 
Table 7

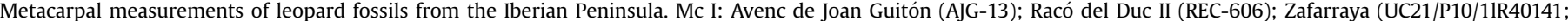

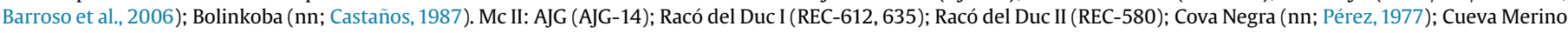

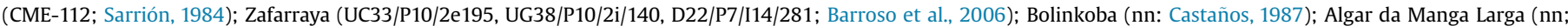

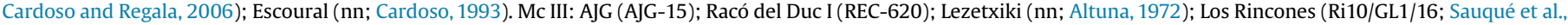

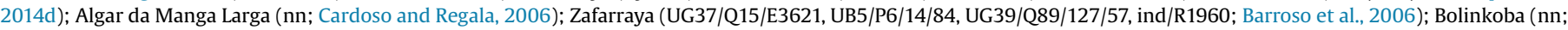

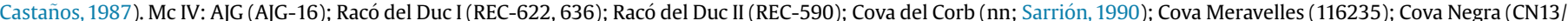

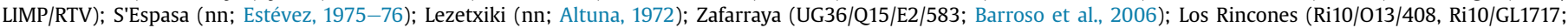

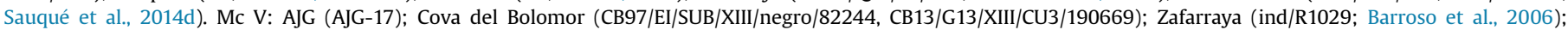
Bolinkoba (nn, Castaños, 1987); Gruta do Caldeirão and Escoural (nn; Cardoso, 1993). [Approximate measurement]. nn (no number).

\begin{tabular}{|c|c|c|c|c|c|c|c|c|c|c|c|c|}
\hline Mc I & AJG & REC II & $\mathrm{BZ}$ & BOK & & & & & & & & \\
\hline GL & 26.3 & 29.9 & 29.3 & 28 & & & & & & & & \\
\hline Bp ap & 13.1 & 15.3 & 11.5 & & & & & & & & & \\
\hline $\mathrm{Bpt}$ & 9.9 & 12.2 & 13.2 & & & & & & & & & \\
\hline SD & 9.4 & 11 & 9.7 & & & & & & & & & \\
\hline Bd ap & 9.6 & 11.5 & 10 & & & & & & & & & \\
\hline $\mathrm{Bd} \mathrm{t}$ & 8.7 & 10.9 & 11.5 & 10.6 & & & & & & & & \\
\hline Mc II & AJG & REC I & REC II & $\mathrm{CN} 1$ & MER & $\mathrm{BZ}$ & BOK & AML & ESC & & & \\
\hline GL & 62.9 & 67.5 & & & 65.1 & $67.6-58.5-66.7$ & $66-66-67.5$ & & 69.3 & & & \\
\hline Bp ap & 18.5 & $17.5-17.1$ & [19.7] & & 17.3 & $20.5-15-19.6$ & & 15.7 & & & & \\
\hline Bp t & 11.6 & $13.1-13$ & [14.2] & & 13.2 & $13-12-14.8$ & & 12.2 & & & & \\
\hline SD & 8.6 & $9.8-9.6$ & & & 10.6 & $10-8.3-9.3$ & $10.4-10-9.2$ & & 9.2 & & & \\
\hline Bd ap & 13.3 & {$[10.5]$} & & & 13.5 & $14.7-10.5-13.7$ & & & & & & \\
\hline Bd t & 13.8 & 14.3 & & 13.6 & 14.7 & $14.3-13-15.7$ & $15.3-15-13.9$ & & & & & \\
\hline Mc III & AJG & REC I & LEZ & LR & AML & $\mathrm{BZ}$ & BOK & & & & & \\
\hline GL & 71.7 & & 84 & 77 & 69.6 & $79.6-79.8-75.3-70.8$ & $72-75.5$ & & & & & \\
\hline Bp ap & 15.4 & & & & 14.3 & $18-17.2-17.7-16.5$ & & & & & & \\
\hline $\mathrm{Bpt}$ & 14.5 & & & & 15.6 & $16.1-18.4-16.6-16.1$ & & & & & & \\
\hline SD & 9.6 & 10.8 & 11.6 & 10.5 & 9 & $10.9-11.7-10.8-10.2$ & $10.7-11.5$ & & & & & \\
\hline Bd ap & 13.3 & 13.9 & & & 11.7 & $15-15.8-15.9-14.2$ & & & & & & \\
\hline $\mathrm{Bd} \mathrm{t}$ & 14.4 & 16.1 & & 15.7 & 13.7 & $15.9-17.2-17.7-14.3$ & $15.5-15.6$ & & & & & \\
\hline Mc IV & AJG & REC I & REC II & COR & MEV & CN3 & ESP & LEZ & $\mathrm{BZ}$ & LR & AML & FUR \\
\hline GL & 69.3 & 73.9 & & & & 68.6 & & 84.5 & 77.8 & $70.5-74.7$ & & 78.8 \\
\hline Bp ap & 15.3 & $14.4-16$ & 17 & 16.6 & 16.1 & 14.2 & 15.8 & & 16.6 & & 14 & \\
\hline Bp t & 11.7 & $11.7-11.9$ & 14.1 & $12.2-13.2$ & 13 & 11.4 & 13.7 & & 12.9 & & 12.5 & \\
\hline SD & 8.4 & 9.8 & & & & 8.2 & & & 10 & $8.7-10.1$ & & 10.6 \\
\hline Bd ap & 12.9 & 13.9 & & & & 12.6 & & & 14.5 & & & \\
\hline Bd t & 12.9 & 14.4 & & & & 12.1 & & & 14.8 & $13.7-13.8$ & & 15.4 \\
\hline Mc V & AJG & BOL & $\mathrm{BOL}$ & $\mathrm{BZ}$ & BOK & CAL & ESC & & & & & \\
\hline GL & 55.9 & & & 59.4 & $64.2-74.6$ & 79.2 & 70.3 & & & & & \\
\hline Bp ap & 14.5 & & & 15.4 & & & & & & & & \\
\hline Bp t & 13.2 & & & 14.6 & & & & & & & & \\
\hline SD & 8.4 & 9.5 & & 9.1 & $9.8-9.2$ & 10.3 & 7.4 & & & & & \\
\hline Bd ap & 12.4 & 12 & 11.9 & 14.2 & & & & & & & & \\
\hline Bd t & 13 & 14 & 12.7 & 12.9 & $14.7-13.8$ & 13.9 & 11.9 & & & & & \\
\hline
\end{tabular}

Table 8

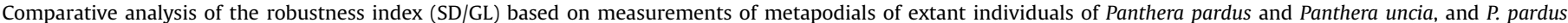
vraonensis with leopard fossils from the Mediterranean Iberia (number of remains of each sample).

\begin{tabular}{|c|c|c|c|c|c|c|c|c|c|}
\hline \multirow{2}{*}{$\frac{\text { Taxa }}{\text { Site }}$} & \multirow{2}{*}{$\frac{\text { P. pardus }}{\text { Extant }}$} & \multirow{2}{*}{$\frac{\text { P. uncia }}{\text { Extant }}$} & \multirow{2}{*}{$\frac{P . p . \text { vraonensis }}{\text { Vraona }}$} & \multicolumn{6}{|c|}{ Panthera pardus } \\
\hline & & & & AJG & REC I & REC II & CN3 & BOL & MER \\
\hline Reference & \multicolumn{3}{|l|}{ Nagel 1999} & \multicolumn{6}{|c|}{ This study } \\
\hline Mc I & $32.64(3)$ & $32.63(8)$ & $37.05(7)$ & 35.74 & & 36.78 & & & \\
\hline Mc II & $11.96(16)$ & $13.22(8)$ & $14.61(4)$ & 13.67 & 14.51 & & & & 16.28 \\
\hline Mc III & $11(16)$ & $12.53(8)$ & $14.59(3)$ & 13.38 & & & & & \\
\hline Mc IV & $10.89(16)$ & $12.16(8)$ & $12.92(5)$ & 12.12 & 13.26 & & 11.95 & & \\
\hline Mc V & $13.56(16)$ & $15.21(8)$ & $15.65(9)$ & 15.02 & & & & & \\
\hline Mt II & $9.33(15)$ & $11.36(8)$ & $12.18(3)$ & 11.58 & & 11.5 & & & \\
\hline Mt III & $10.65(17)$ & $10.81(8)$ & $13.45(2)$ & 12.93 & 13.48 & & & 12.31 & 13.18 \\
\hline Mt IV & $9.52(16)$ & $10.87(8)$ & $12.15(3)$ & & & 11.83 & & & \\
\hline Mt V & $8.67(15)$ & $9.71(8)$ & $9.97(3)$ & & 10.27 & 10.39 & & & \\
\hline
\end{tabular}

remains. The cranium shows a 2 -cm depression between the frontal and nasal bones. The right nasal bone appears to be partially sunken and has microfissures. The body of the left scapula has several fractures and cracks that have caused lateral torsion of the bone. All these cases correspond to an incomplete fracture. The left radius and ulna present complete oblique-spiral fractures on the proximal diaphysis that occurred in fresh bone, which coincide anatomically and were caused at the same time. A similar fracture can be seen on the distal diaphysis of the left ulna. There is a fracture at the base of the spinous process of the first thoracic vertebra. Seven rib fragments present transverse fractures. No bone remodelling processes are observed, so these are perimortem 
Table 9

Cervical vertebrae measurements of leopard fossils from the Iberian Peninsula. Vc I: Avenc de Joan Guitón (AJG-54); Abric Romaní (nn; Cáceres et al., 1993). Vc II: AJG (AJG-55); Bolinkoba (nn; Castaños, 1987).Vc III: AJG (AJG-56); Abric Romaní (nn; Cáceres et al., 1993). Vc IV: AJG (AJG-57). Vc V: AJG (AJG-58); Abric Romaní (nn; Cáceres et al., 1993). Vc VI: AJG (AJG-59). Vc VII: AJG (AJG-60). [Approximate measurement]. nn (no number).

\begin{tabular}{|c|c|c|}
\hline 1st cervical & $\mathrm{AJG}$ & ARO \\
\hline GL & 47.8 & 45.3 \\
\hline \multicolumn{3}{|l|}{ GB } \\
\hline Lad & 21.4 & [22.5] \\
\hline $\mathrm{BFcr}$ & 41.2 & 43.8 \\
\hline BFcd & 39.7 & 47.8 \\
\hline $\mathrm{H}$ & 31 & 28.7 \\
\hline 2nd cervical & AJG & BOK \\
\hline $\mathrm{H}$ & 54.7 & \\
\hline LAPa & 54.8 & \\
\hline LCDe & [55] & \\
\hline $\mathrm{BFcr}$ & [39.1] & 45.5 \\
\hline 3rd cervical & $\mathrm{AJG}$ & ARO \\
\hline GLPa & 37.4 & \\
\hline Bpacr & & 42.1 \\
\hline Bpacd & 39.8 & 43 \\
\hline \multicolumn{3}{|l|}{ PL } \\
\hline 4th cervical & $\mathrm{AJG}$ & \\
\hline \multicolumn{3}{|l|}{ GLPa } \\
\hline \multicolumn{3}{|l|}{ Bpacr } \\
\hline Bpacd & [55.2] & \\
\hline PL & 30.1 & \\
\hline 5th cervical & AJG & ARO \\
\hline \multicolumn{3}{|l|}{ GLPa } \\
\hline Bpacr & & 43.5 \\
\hline Bpacd & 39.5 & 43.2 \\
\hline 6th cervical & AJG & \\
\hline GLPa & 31.7 & \\
\hline Bpacr & 39.8 & \\
\hline Bpacd & 39.9 & \\
\hline 7th cervical & AJG & \\
\hline GLPa & 32.2 & \\
\hline Bpacr & 40 & \\
\hline Bpacd & 35.4 & \\
\hline
\end{tabular}

alterations that occurred as a result of the animal falling (Bartosiewicz, 2013). There is no evidence of bite marks on the remains, so no carnivore had access to the carcass and fed on it.

The skeleton was sent for radiocarbon dating to VERAlaboratorium (Vienna), but this was impossible due to the very low collagen content of the fossils. This is common in other carnivores found on the surface inside caves with little sedimentation, such as the specimens found at Algar da Manga Larga (Cardoso and Regala, 2006) and Los Rincones (Sauqué et al., 2014b).

\subsubsection{Cova de les Malladetes}

This cave is situated in the vicinity of Barx (Valencia), 631 masl in the Mondúver mountains in the south of the province of Valencia. This site has been the subject of archaeological research during the 20th century (Fortea and Jordá, 1976; Davidson, 1989) and beginning of the 21st century (De la Peña, 2013). Four leopard specimens (Table 1) were found at the basal levels of the 1940s excavations (Sector D, E, I): pelvis (Fig. 4A), $\mathrm{m}_{1}$ germinal (Fig. 4B) and two first phalanges (Fig. 4C, D). The remains are those of two individuals (an adult and a juvenile aged $<1$ year) and they are linked to lithic materials and the bone industry of the Aurignacian and the faunal remains of different species, including Capra pyrenaica. The archaeozoological and taphonomic study of the fauna is still in progress, but it is seems likely that the accumulation is mainly due to the action of carnivores, with minor human involvement. As regards the chronology of the lower levels, AMS dating for layer 12 of sector $\mathrm{E}(25.1 \mathrm{ky})$ corresponds to the Gravettian period in the region (Arsuaga et al., 2002), above the level where the leopard remains lay. There is another date for level XII (eastern section of the 1970s excavations), with associated lithic material, with a result of $29.6 \pm 0.5$ ky (Fortea and Jordá, 1976).

\subsubsection{Cova del Racó del Duc}

This cave is located in a ravine with very vertical walls in the vicinity of Vilallonga, next to the river Serpis at 200 masl, in the south of the province of Valencia. It has a small vestibule that leads to an inner chamber, at the back of which there is a pit (JuanCabanilles, 1991, 1997). The leopard materials, which were recovered by I. Sarrión in 1970, come from an area of the vestibule (REC I) and the bottom of the pit (REC II).

REC I consists of 40 well-preserved ossified remains (dark colour) belonging to two adult individuals, one larger than the other (Table 1; Fig. 5A-AN). They were located in level G-F of sector $B$ associated with $C$. pyrenaica remains which may have been accumulated by leopards, although the taphonomic study is yet to be concluded. Human presence in the cave at the end of the Solutrean corresponds to levels C, D and E of sector A, which means that the leopard-Spanish ibex association of levels G-F precedes the archaeological level. A very worn canine from the largest individual indicates that its age was 7-10 years (Stander, 1997).

REC II consists of 38 poorly preserved ossified remains (light colour) of a large individual (Table 1; Fig. 6A-AO), published preliminarily by Sarrión (1976). The upper incisors are worn and indicate an age of 3-4 years (Stander, 1997).

\subsubsection{Other sites in the Valencia area}

New leopard remains are presented from archaeological and palaeontological sites in the Valencia area (Table 1).

From an Early Late Pleistocene (MIS 5e) level of Cova del Bolomor (Tavernes de la Valldigna, Valencia), an ossified distal fragment of Mc V has been identified (Fig. 7A) (Fernández, 2004, 2007). Recently, another three remains were recovered from level XIII of the northern sector (Middle Pleistocene, MIS 7, 190-200 ky): distal half of Mc V, complete Mt III and distal fragment of Mt IV, all of which were ossified and probably belonged to the same individual (Fig. 7B-D).

At Cova Negra (Xàtiva, Valencia), leopard remains were identified by various investigators (Royo, 1942; Pérez, 1977; Martínez, 1996, 2009). The sequence of this Middle Palaeolithic site (Villaverde, 2009) is being studied and its origin may be put back to the end of the Middle Pleistocene (MIS 7-6) and beginning of the Late Pleistocene (MIS 5e), with its maximum development during the Middle Pleistocene (Villaverde et al., 2014). Three new ossified remains are presented from disturbed levels of the 2013 excavation: Mc IV, calcaneus and second phalanx (Fig. 7E-G), and also Mt V (Fig. 7H) and two premolars (P4 and p4) published (Pérez, 1977) but not described (Fig. 7I-J).

In the province of Castellón, two new remains of leopard have been found without any context: a proximal fragment of ulna (Fig. 7K) from Cau d'En Borràs (Orpesa), where there was already a previous citation (Carbonell et al., 1979; Fernández, 2004), and a first phalanx (Fig. 7L) from Cueva Horadada (Azuébar).

A leopard Mc IV (Fig. 7M) without any context from Cova de les Meravelles (Alzira, Valencia) is associated with fauna of natural origin (Breuil and Obermaier, 1914).

\section{Systematic and morphometric study of the new leopard remains}

Order Carnivora Bowdich, 1821

Family Felidae Fischer, 1817

Genus Panthera Oken, 1816

Species $P$. pardus Linnaeus, 1758 
Table 10

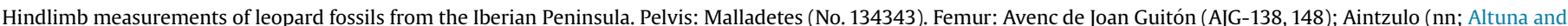

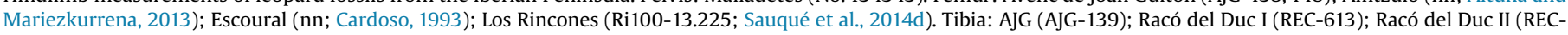

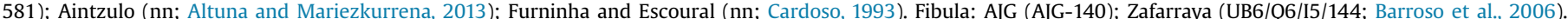

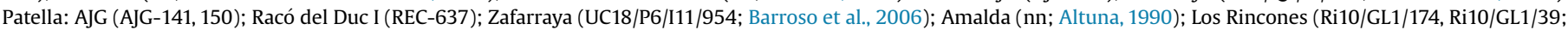

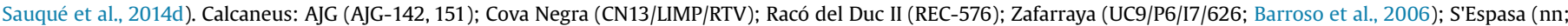

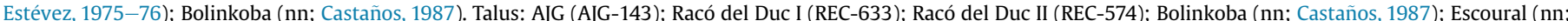

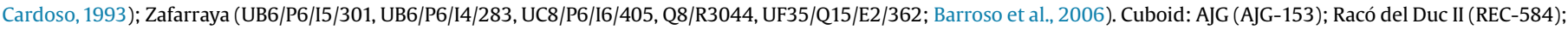

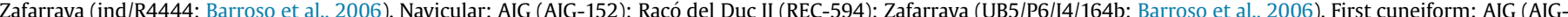

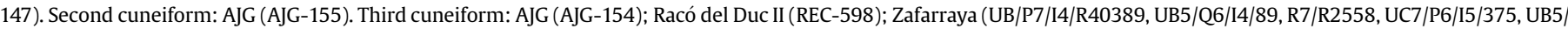
P6/I4/115; Barroso et al., 2006). [Approximate measurement]. nn (no number).

\begin{tabular}{|c|c|c|c|c|c|c|c|c|}
\hline Pelvis & MAL & & & & & & & \\
\hline LAR & 28.2 & & & & & & & \\
\hline LA & 30.7 & & & & & & & \\
\hline SB & 13.9 & & & & & & & \\
\hline GL & [180] & & & & & & & \\
\hline L ilion & [106] & & & & & & & \\
\hline Femur & AJG & AIN & ESC & LR & & & & \\
\hline GL & & 261.5 & 266.7 & & & & & \\
\hline \multicolumn{9}{|l|}{ Bp ap } \\
\hline $\mathrm{Bp} \mathrm{t}$ & $51.2-49.1$ & 54.8 & & & & & & \\
\hline SD & 21.5 & 20.6 & 21.7 & & & & & \\
\hline \multicolumn{9}{|l|}{$\mathrm{Bd}$ ap } \\
\hline $\mathrm{Bd} \mathrm{t}$ & & 51.9 & 51.9 & 44.3 & & & & \\
\hline DC & 24.6 & 27.6 & & & & & & \\
\hline Tibia & AJG & REC I & REC II & AIN & FUR & ESC & LR & \\
\hline GL & & [232] & & [239] & & & & \\
\hline Bp ap & 47.4 & 56.4 & & & & & & \\
\hline $\mathrm{Bpt}$ & [47.2] & 54.4 & & 56.2 & & & & \\
\hline SD & 18 & 21.9 & & 19.3 & 22.2 & 24.9 & & \\
\hline $\mathrm{Bd}$ ap & 20.6 & [26.8] & [27.6] & & & & & \\
\hline $\mathrm{Bd} \mathrm{t}$ & 35 & [35.2] & [35.9] & & 38.4 & $42.1-43.3$ & $39.9-42.4$ & \\
\hline Fibula & AJG & $\mathrm{BZ}$ & & & & & & \\
\hline GL & & 220 & & & & & & \\
\hline Bp ap & & 13.6 & & & & & & \\
\hline Bp t & & 24.6 & & & & & & \\
\hline Bd ap & 19.6 & 11.6 & & & & & & \\
\hline $\mathrm{Bd} \mathrm{t}$ & 9.9 & 5.5 & & & & & & \\
\hline Patella & AJG & REC I & $\mathrm{BZ}$ & AMA & LR & & & \\
\hline GL & 32.1 & 35.6 & 31.5 & 38.5 & $36.2-36.6$ & & & \\
\hline GB & $23.5-23.6$ & 27.6 & 24 & 27.5 & $27.5-27.5$ & & & \\
\hline Calcaneus & AJG & CN3 & REC II & $\mathrm{BZ}$ & ESP & ВОК & FUR & LR \\
\hline Bp ap & $20.6-21.2$ & 25.7 & & & & & & \\
\hline $\mathrm{Bp} \mathrm{t}$ & 17.4 & 25.3 & & & & & & \\
\hline GL & $60.4-[60]$ & 62.1 & 71.8 & 69.3 & 67.5 & $79-67-70.5$ & 59.8 & 72 \\
\hline DT & $26.6-25.9$ & 26.1 & 31.5 & 33.6 & & $36.5-33.5-35$ & 26.1 & 33.1 \\
\hline KD & $11.1-11.7$ & 11.2 & 13 & & & & & \\
\hline $\mathrm{KD} / \mathrm{GL}$ & $18-19.5$ & 18 & 18 & & & & & \\
\hline Talus & AJG & REC I & REC II & BOK & ESC & $\mathrm{BZ}$ & LR & \\
\hline GL ext & & 37.6 & 41.9 & $38.2-38.6$ & 40.7 & $41.7-36.5-36.8-35.8-40.2$ & 39.6 & \\
\hline GL int & & 26.4 & 27.9 & & & $29.9-25.7-25.1-26.1-27.5$ & & \\
\hline B troclea & 22.1 & 21.9 & 25.8 & & & $22.8-21-17.9-19.3-21.3$ & & \\
\hline B neck & & 17.1 & 20.2 & & & $17.2-16.9-15.1-16-16.1$ & & \\
\hline $\mathrm{B}$ head & & 21.6 & 24.6 & & & $21.9-21.7-20.2-20.2-23.1$ & & \\
\hline Cuboid & AJG & REC II & $\mathrm{BZ}$ & & & & & \\
\hline DAP & 17.7 & 20.1 & 18.2 & & & & & \\
\hline DT & 18.2 & 21.1 & 18.4 & & & & & \\
\hline $\mathrm{H}$ & 18.2 & 20.1 & 18.6 & & & & & \\
\hline Navicular & AJG & REC II & $\mathrm{BZ}$ & & & & & \\
\hline DAP & 23.4 & 29.7 & 21.9 & & & & & \\
\hline DT & [19.9] & 25.5 & 19 & & & & & \\
\hline $\mathrm{H}$ & 17.9 & 18.8 & 16.6 & & & & & \\
\hline Cuneif. I & AJG & & & & & & & \\
\hline $\mathrm{H}$ & 14.9 & & & & & & & \\
\hline Cuneif. II & AJG & & & & & & & \\
\hline DAP & 13.3 & & & & & & & \\
\hline DT & 7.1 & & & & & & & \\
\hline $\mathrm{H}$ & 8.2 & & & & & & & \\
\hline Cuneif. III & AJG & REC II & $\mathrm{BZ}$ & & & & & \\
\hline DAP & 24.7 & 31.4 & $27.1-28.7-28.2-26.7-24.3$ & & & & & \\
\hline DT & 14.9 & 17.7 & $16.4-16.1-16.1-14.4-14.8$ & & & & & \\
\hline $\mathrm{H}$ & 12.6 & 15.4 & $13-15-13.5-13.2-12.4$ & & & & & \\
\hline
\end{tabular}


Table 11

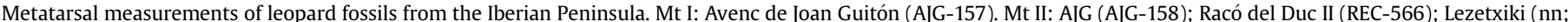

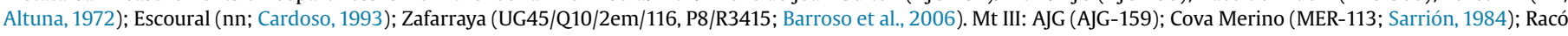

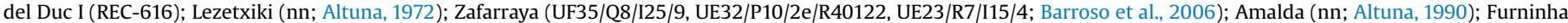

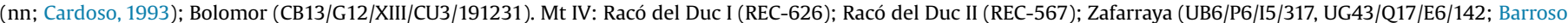

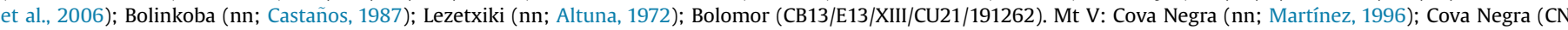

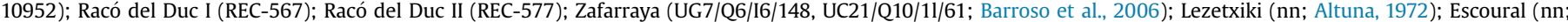
Cardoso, 1993); Los Rincones (Ri10/GL1/18). [Approximate measurement]. nn (no number).

\begin{tabular}{|c|c|c|c|c|c|c|c|c|}
\hline Mt I & $\mathrm{AJG}$ & & & & & & & \\
\hline GL & 19.4 & & & & & & & \\
\hline Bp ap & 11 & & & & & & & \\
\hline Bp t & 8 & & & & & & & \\
\hline SD & 4.4 & & & & & & & \\
\hline Mt II & AJG & REC II & LEZ & ESC & $\mathrm{BZ}$ & & & \\
\hline GL & 76.8 & 87.8 & & $86.9-89.4$ & $60-77.9$ & & & \\
\hline Bp ap & 16.4 & 19.8 & & & $17.4-16.8$ & & & \\
\hline Bp t & 10.2 & 13.3 & & & $12-10.2$ & & & \\
\hline SD & 8.9 & 10.1 & 9.3 & $9.8-10.2$ & $9.8-9.2$ & & & \\
\hline Bd ap & [11.5] & 14.6 & & & $13.3-13.8$ & & & \\
\hline$B d t$ & 12.5 & 155 & & $14.1-15.9$ & $13.3-16.8$ & & & \\
\hline Mt III & AJG & MER & REC I & LEZ & $\mathrm{BZ}$ & AMA & FUR & BOL \\
\hline GL & 88.1 & 91.8 & 91.2 & 101.2 & 99.7-97.8-90.9 & 104 & 91.5 & 88.5 \\
\hline Bp ap & & 19.6 & 21 & & $22.5-22.5-18.6$ & & & \\
\hline Bp t & 15.5 & 16.7 & 17 & & $17.3-17.8-16.6$ & & & 15 \\
\hline SD & 11.4 & 12.1 & 12.3 & 13 & $11.7-12.9-12.8$ & 15.3 & 12.5 & 10.9 \\
\hline Bd ap & 13.9 & 15 & 14.8 & 3 & 15.9-15.2-15 & & & 13.2 \\
\hline $\mathrm{Bd} \mathrm{t}$ & 15.4 & 16.2 & 17.2 & 3 & $16.9-15.8-15.8$ & & 16.5 & [13.9] \\
\hline Mt IV & REC I & REC II & $\mathrm{BZ}$ & BOK & LEZ & $\mathrm{BOL}$ & & \\
\hline GL & & 99.7 & $96.2-100.4$ & 94 & [104] & & & \\
\hline Bp ap & 17 & 21.5 & $18.1-20.2$ & & & & & \\
\hline Bp t & 15.6 & 17.7 & $17.9-17$ & & & & & \\
\hline SD & 10.9 & 11.8 & $11.2-11.6$ & 10.5 & 11.7 & & & \\
\hline Bd ap & & 15.8 & $15.8-15.5$ & & & 12.5 & & \\
\hline $\mathrm{Bd} \mathrm{t}$ & & 15.7 & $15.4-15.3$ & 14.7 & & [12.5] & & \\
\hline Mt V & $\mathrm{CN} 2$ & CN3 & REC I & REC II & $\mathrm{BZ}$ & LEZ & ESC & LR \\
\hline GL & & & 82.7 & 88.5 & $84.7-91.7$ & [97]-83.2 & 88.2 & 87.6 \\
\hline Bp ap & & 14 & 12.6 & 11.6 & $13.9-13$ & & & \\
\hline $\mathrm{Bpt}$ & 13.4 & 11.4 & 15.7 & 17.4 & $15.5-16.5$ & & & \\
\hline SD & & 9.1 & 8.5 & 9.2 & $9.0-9.0$ & 9.3 & 8.2 & 8.3 \\
\hline Bd ap & & & 13.7 & 15.1 & $14-14.2$ & & & \\
\hline $\mathrm{Bd} \mathrm{t}$ & & & 12.4 & 13.6 & $12.9-14$ & & 12.4 & 13.1 \\
\hline
\end{tabular}

Table 12

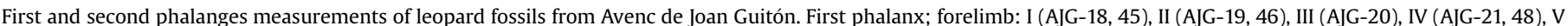

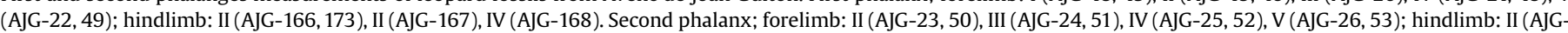
176), III (AJG-170, 177), IV (AJG-171), V (AJG-172). [Approximate measurement].

\begin{tabular}{|c|c|c|c|c|c|c|c|}
\hline 1st phalanx AJG & & GL & Bp ap & $\mathrm{Bp} \mathrm{t}$ & SD & Bd ap & $B d t$ \\
\hline \multirow[t]{5}{*}{ Fore limb } & I & $19.3-19.1$ & $9.7-9.8$ & $12.5-12.7$ & $10.2-9.6$ & $8.9-8.1$ & $10.9-10$ \\
\hline & II & $32.7-32.1$ & $10.8-10.8$ & $12.7-11.7$ & $7.2-7.5$ & $7.6-8.1$ & $9.6-9.9$ \\
\hline & III & 38.4 & 10.8 & 13.8 & $9.3-8.7$ & $8.5-8.5$ & $10.4-10.3$ \\
\hline & IV & $36.8-36.8$ & $10.4-11.8$ & $13.4-13.2$ & $8.5-9.2$ & $8.4-8.9$ & $10.3-10.8$ \\
\hline & $\mathrm{V}$ & $33-32.3$ & $10.4-10.8$ & $12.9-11.8$ & $7.9-7.4$ & $7.7-8.5$ & $9.6-9.8$ \\
\hline \multirow[t]{3}{*}{ Hind limb } & II & $33.4-[33.2]$ & 11.1-[9] & 13 & $9-9.2$ & $8.9-8.3$ & $10.5-10.4$ \\
\hline & III & 37.1 & $11.7-11.5$ & $14.2-13.7$ & $10.6-9.8$ & 8.4 & {$[11.8]$} \\
\hline & IV & [35.9] & 11 & [13.1] & 9.5 & $8.7-8.7$ & $11.2-11.4$ \\
\hline 2nd phalanx AJG & & GL & Bp ap & $\mathrm{Bpt}$ & SD & Bd ap & $\mathrm{Bd} \mathrm{t}$ \\
\hline \multirow[t]{4}{*}{ Fore limb } & II & $25.4-25.6$ & $11.8-11.4$ & $11.5-11.3$ & $6.4-6.2$ & $8-8.6$ & $10.5-10.6$ \\
\hline & III & $30-30.2$ & $10.5-11.3$ & $10.7-11.2$ & $6.5-6.7$ & $8.3-8.2$ & $10.4-10.5$ \\
\hline & IV & $28.7-28.8$ & $10.2-10.4$ & $10.7-10.8$ & $6.5-6.5$ & $7.6-8.2$ & $9.6-10.1$ \\
\hline & $\mathrm{V}$ & $24-23.2$ & $10-9.9$ & $10.4-10.5$ & $7.3-7.2$ & $7.3-7.5$ & $9.2-9.5$ \\
\hline \multirow[t]{4}{*}{ Hind limb } & II & 24 & 10 & [11.3]-10.8 & 7.9 & 7.3 & 9.6 \\
\hline & III & $27.6-27.2$ & [10.4]-[10] & {$[10]-[11.1]$} & $6.7-6.7$ & $8-8.2$ & $10.4-10.4$ \\
\hline & IV & 27 & 10.2 & 10.9 & 6.9 & 8.2 & 10.1 \\
\hline & $\mathrm{V}$ & 22.3 & [9.2]-9.5 & $10.6-10.9$ & 7.5 & 7.5 & 9.3 \\
\hline
\end{tabular}

\subsection{Avenc de Joan Guitón}

\subsubsection{Cranium}

The cranium from AJG has a convex nasal profile in lateral view (Fig. 8C), a feature of P. pardus and Puma concolor, whereas in Panthera uncia and Panthera onca it is concave. The auditory bulla presents a flat projection of the ectotympanic bone (Fig. 8B), which is typical of $P$. pardus and $P$. onca. These features enable us to attribute the cranium found at AJG to the leopard (Sims, 2005, 2012).

The neurocranium shows a globular profile that projects backward at the level of the occipital bone (Fig. 8C, F), which is wider 
Table 13

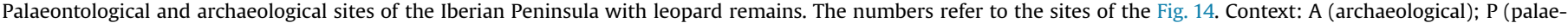

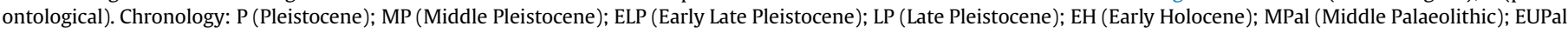

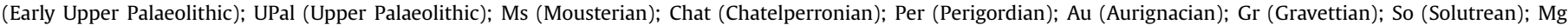
(Magdalenian); Az (Azilian); Mes (Mesolithic).

\begin{tabular}{|c|c|c|c|c|c|c|c|c|c|}
\hline $\mathrm{N}^{\circ}$ & Site & Level & masl & Context & Chronology & Taxon & NISP & MNI & References \\
\hline 1 & Cueva Eirós (Triacastela, Lugo) & 2 & 780 & A & LP (EUPal) & P. pardus & \multicolumn{2}{|c|}{ Presence } & $\begin{array}{l}\text { De Lombera-Hermida } \\
\text { et al., } 2014\end{array}$ \\
\hline 2 & $\begin{array}{l}\text { Valdavara } 3 \text { (Becerreá, } \\
\text { Lugo) }\end{array}$ & Depósito & 600 & $\mathrm{P}$ & $\begin{array}{l}\text { ELP } \\
(120-100 \mathrm{ka})\end{array}$ & Leopard & \multicolumn{2}{|c|}{ Presence } & Alonso et al., 2014 \\
\hline 3 & $\begin{array}{l}\text { Cueva de las Caldas } \\
\text { (Priorio, Asturias) }\end{array}$ & - & 160 & A & LP (So.) & Leopard & \multicolumn{2}{|c|}{ Presence } & Corchón, 2014 \\
\hline 4 & $\begin{array}{l}\text { Cueva de la Güelga } \\
\text { (Cangas de Onís, Asturias) }\end{array}$ & D, level 9 & 200 & A & $\begin{array}{l}\text { LP } \\
\text { (Ms., 45-48 ka) }\end{array}$ & P. pardus & \multicolumn{2}{|c|}{ Presence } & Menéndez et al., 2014 \\
\hline 5 & $\begin{array}{l}\text { Cueva de La Riera } \\
\text { (Posada de Llanes, Asturias) }\end{array}$ & Azilian & 30 & A & $\mathrm{EH}(\mathrm{Az})$. & P. pardus & \multicolumn{2}{|c|}{ Presence } & $\begin{array}{l}\text { Vega del Sella, 1930; } \\
\text { Fraga, 1958; } \\
\text { Álvarez-Laó, } 2003\end{array}$ \\
\hline 6 & $\begin{array}{l}\text { Cueva de Jou Puerta } \\
\text { (Llanes, Asturias) }\end{array}$ & - & 28 & $\mathrm{P}$ & $\begin{array}{l}\text { LP (MIS 3, } \\
36.6-30.2 \mathrm{ka})\end{array}$ & cf. P. pardus & 1 & 1 & Álvarez-Laó, 2014 \\
\hline 7 & $\begin{array}{l}\text { Cueva de Llonín } \\
\text { (Peñamera Alta, Asturias) }\end{array}$ & $\begin{array}{l}\text { VI (conjunto II)VI } \\
\text { (conjunto II)VI } \\
\text { (conjunto II) }\end{array}$ & ca. 200 & A & LP (EUPal) & P. pardus & 30 & 1 & $\begin{array}{l}\text { Fortea et al., 1992, 1995, } \\
\text { 1999; De la Rasilla and } \\
\text { Santamaría, 2011-2012 }\end{array}$ \\
\hline 8 & $\begin{array}{l}\text { Cueva de Hornos de la } \\
\text { Peña (S. F. de Buelna, } \\
\text { Cantabria) }\end{array}$ & $\mathrm{E}$ & 280 & A & LP (Au.) & P. pardus & 5 & 1 & Yravedra, 2010b \\
\hline 9 & $\begin{array}{l}\text { Cueva del Castillo } \\
\text { (Puente Viesgo, Cantabria) }\end{array}$ & 22 & 190 & A & LP (Ms.) & Felis pardus & \multicolumn{2}{|c|}{ Presence } & Cabrera, 1984 \\
\hline 9 & $\begin{array}{l}\text { Cueva del Castillo } \\
\text { (Puente Viesgo, Cantabria) }\end{array}$ & Must A & & A & LP (Ms.) & P. pardus & 3 & 1 & Dari, 1999; Quesada, 2006 \\
\hline 9 & $\begin{array}{l}\text { Cueva del Castillo } \\
\text { (Puente Viesgo, Cantabria) }\end{array}$ & $18-14$ & & A & LP (Au.-Per.) & P. pardus & \multicolumn{2}{|c|}{ Presence } & Cabrera, 1984 \\
\hline 9 & $\begin{array}{l}\text { Cueva del Castillo } \\
\text { (Puente Viesgo, Cantabria) }\end{array}$ & Aur. B & & A & LP (Au.) & P. pardus & 5 & 2 & Dari, 1999; Quesada, 2006 \\
\hline 9 & $\begin{array}{l}\text { Cueva del Castillo } \\
\text { (Puente Viesgo, Cantabria) }\end{array}$ & 12_14 & & A & LP (Gr.) & P. pardus & 7 & 2 & $\begin{array}{l}\text { Bernaldo de Quirós } \\
\text { et al., } 2014\end{array}$ \\
\hline 10 & $\begin{array}{l}\text { Cueva de El Juyo } \\
\text { (Igollo, Cantabria) }\end{array}$ & 4 & 35 & A & LP (Mg.) & P. pardus & 1 & 1 & Klein and Cruz Uribe, 1985 \\
\hline 11 & $\begin{array}{l}\text { Cueva Morín (Villaescusa, } \\
\text { Cantabria) }\end{array}$ & $5 b-5 c$ & 50 & A & LP (Au.) & P. pardus & 1 & 1 & Altuna, 1971, 1994 \\
\hline 12 & $\begin{array}{l}\text { Cueva de El Mirón (Ramales } \\
\text { de la Victoria, Cantabria) }\end{array}$ & 108 & 260 & A & LP (Mg.) & P. pardus & 1 & 1 & Marín-Arroyo, 2009 \\
\hline 13 & $\begin{array}{l}\text { Cueva de las Pajucas } \\
\text { (Lanestosa, Vizcaya) }\end{array}$ & Infra-Eneolithic & 400 & A & $\begin{array}{l}\text { LP/EH? } \\
\text { (UPal/Mes?) }\end{array}$ & P. pardus & 1 & 1 & Altuna, 1972 \\
\hline 14 & $\begin{array}{l}\text { Covacho de Arenillas } \\
\text { (Islares, Cantabria) }\end{array}$ & II & 15 & A & $\mathrm{LP}(\mathrm{Au})$. & P. pardus & \multicolumn{2}{|c|}{ Presence } & $\begin{array}{l}\text { Bohigas and Muñoz, 2002; } \\
\text { Rasines del Río et al., } 2011\end{array}$ \\
\hline 15 & $\begin{array}{l}\text { Abrigo del Cuco (Castro } \\
\text { Urdiales, Cantabria) }\end{array}$ & XIII & 43 & A & LP (Au.) & P. pardus & 1 & 1 & $\begin{array}{l}\text { Castaños and Castaños, 2007; } \\
\text { Rasines del Río et al., } 2011\end{array}$ \\
\hline 16 & $\begin{array}{l}\text { Cueva de Arlanpe } \\
\text { (Lemoa, Vizcaya) }\end{array}$ & $3,4, \mathrm{D}$ & 204 & A & $\begin{array}{l}\text { MP (MPal, } \\
180 \mathrm{ka})\end{array}$ & P. cf. pardus & 5 & 3 & Arceredillo et al., 2013 \\
\hline 16 & $\begin{array}{l}\text { Cueva de Arlanpe } \\
\text { (Lemoa, Vizcaya) }\end{array}$ & II & & A & LP (So.) & $\begin{array}{l}\text { Panthera } \\
\text { sp. }\end{array}$ & 1 & 1 & Arceredillo et al., 2013 \\
\hline 17 & $\begin{array}{l}\text { Cueva de Atxuri } \\
\text { (Mañaria, Vizcaya) }\end{array}$ & $\mathrm{V}-\mathrm{VII}$ & 188 & A & LP (UPal) & P. pardus & 1 & 1 & Castaños, 1987 \\
\hline 18 & $\begin{array}{l}\text { Cueva de Oyalkoba } \\
\text { (Abadiano, Vizcaya) }\end{array}$ & V & ca. 410 & A & LP (UPal) & P. pardus & 1 & 1 & Castaños, 1987 \\
\hline 19 & $\begin{array}{l}\text { Cueva de Bolinkoba } \\
\text { (Abadiano, Vizcaya) }\end{array}$ & VI & 430 & A & LP (Gr.) & P. pardus & 79 & - & Castaños, 1983, 1987 \\
\hline 19 & $\begin{array}{l}\text { Cueva de Bolinkoba } \\
\text { (Abadiano, Vizcaya) }\end{array}$ & IV $-\mathrm{V}$ & & A & LP (So.) & P. pardus & 49 & - & Castaños, 1983, 1987 \\
\hline 19 & $\begin{array}{l}\text { Cueva de Bolinkoba } \\
\text { (Abadiano, Vizcaya) }\end{array}$ & III & & A & LP (Mg.) & P. pardus & 14 & - & Castaños, 1983, 1987 \\
\hline 20 & $\begin{array}{l}\text { Cueva de Axlor } \\
\text { (Dima, Vizcaya) }\end{array}$ & III-I/D & 300 & A & LP (Ms./Au.) & P. pardus & 1 & 1 & $\begin{array}{l}\text { Altuna, 1980, 1994; } \\
\text { Castaños, 2005 }\end{array}$ \\
\hline 21 & $\begin{array}{l}\text { Cueva de Arrillor } \\
\text { (Murua, Álava) }\end{array}$ & Lmc & 710 & A & $\begin{array}{l}\text { LP (Ms., } \\
37,1 \mathrm{ka})\end{array}$ & P. pardus & 4 & - & Castaños, 2005 \\
\hline 22 & $\begin{array}{l}\text { Cueva de Praileaitz I } \\
\text { (Deba, Guipúzcoa) }\end{array}$ & Exterior & 55 & $\mathrm{P}$ & LP & P. pardus & 7 & - & Castaños, 2010 \\
\hline 22 & $\begin{array}{l}\text { Cueva de Praileaitz I } \\
\text { (Deba, Guipúzcoa) }\end{array}$ & Vestíbulo & & A & $\begin{array}{l}\text { LP (Mg., } \\
15,4 \mathrm{ka})\end{array}$ & P. pardus & 6 & - & Castaños, 2010 \\
\hline 23 & $\begin{array}{l}\text { Cueva de Astigarraga } \\
\text { (Deba, Guipúzcoa) }\end{array}$ & II & 425 & A & $\begin{array}{l}\text { LP (So., } \\
16,9 \mathrm{ka})\end{array}$ & P. pardus & 2 & 1 & $\begin{array}{l}\text { Castaños, 2010; } \\
\text { Villaluenga et al., 2012a }\end{array}$ \\
\hline 24 & $\begin{array}{l}\text { Cueva de Ekain } \\
\text { (Deba, Guipúzcoa) }\end{array}$ & $\mathrm{X} \mathrm{a}$ & 90 & A & LP (Chat.) & P. pardus & 3 & 1 & $\begin{array}{l}\text { Altuna and Mariezkurrena, } \\
\text { 1984; Altuna, 1994; } \\
\text { Villaluenga et al., 2012b }\end{array}$ \\
\hline 24 & $\begin{array}{l}\text { Cueva de Ekain } \\
\text { (Deba, Guipúzcoa) }\end{array}$ & IX b & & A & LP (Au.) & P. pardus & 1 & 1 & $\begin{array}{l}\text { Altuna and Mariezkurrena, } \\
\text { 1984; Altuna, } 1994\end{array}$ \\
\hline
\end{tabular}


Table 13 (continued)

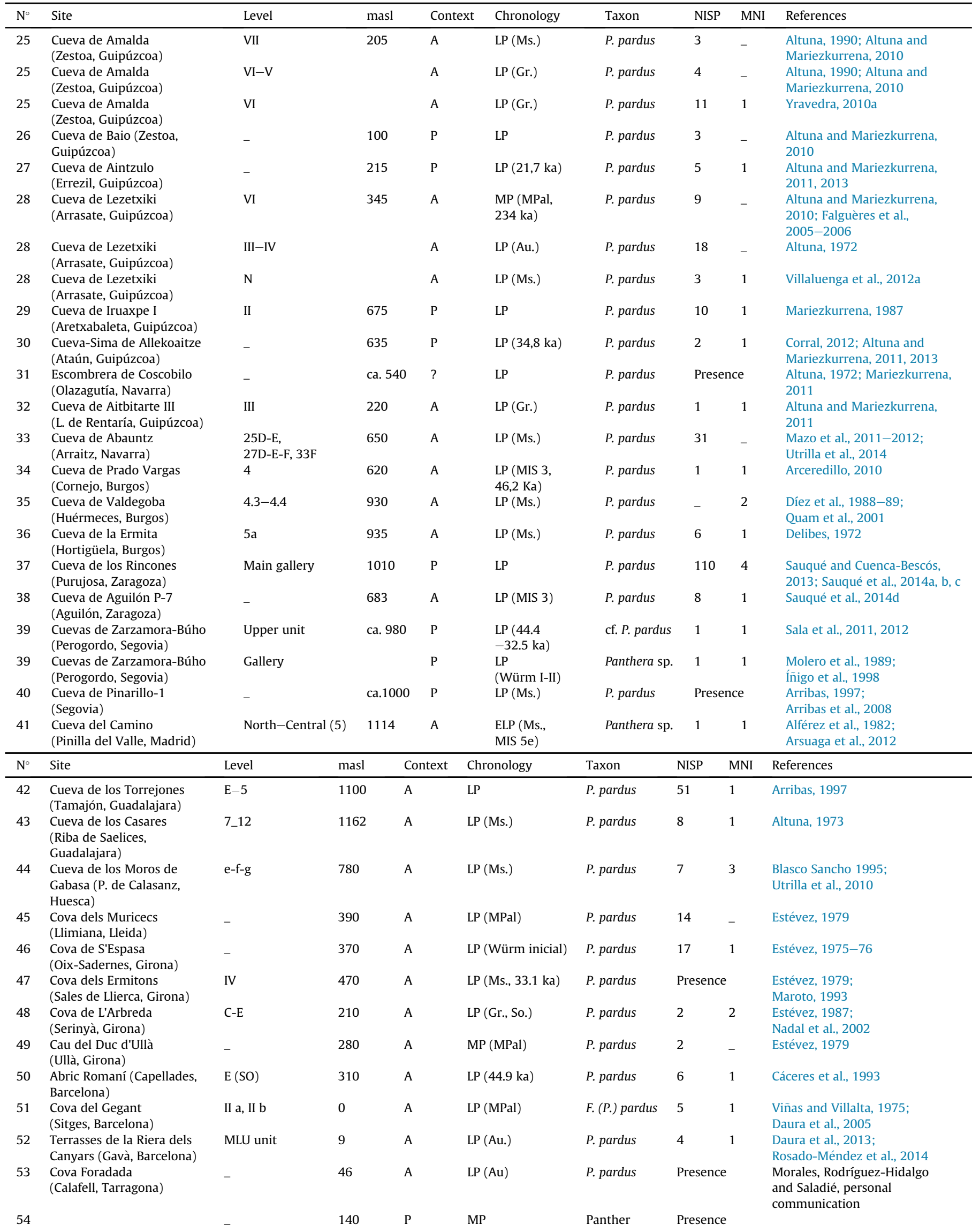


Table 13 (continued)

\begin{tabular}{|c|c|c|c|c|c|c|c|c|c|}
\hline $\mathrm{N}^{\circ}$ & Site & Level & masl & Context & Chronology & Taxon & NISP & MNI & References \\
\hline & $\begin{array}{l}\text { Cau d'En Borràs } \\
\text { (Orpesa, Castelló) }\end{array}$ & & & & & & & & $\begin{array}{l}\text { Carbonell et al., 1979; Fernández, } \\
2004\end{array}$ \\
\hline 54 & $\begin{array}{l}\text { Cau d'En Borràs } \\
\text { (Orpesa, Castelló) }\end{array}$ & - & & $\mathrm{P}$ & MP & P. pardus & 1 & 1 & This study \\
\hline 55 & $\begin{array}{l}\text { Cueva Horadada } \\
\text { (Azuebar, Castelló) }\end{array}$ & - & ca.300 & A & LP (UPal) & P. pardus & 1 & 1 & This study \\
\hline 56 & $\begin{array}{l}\text { Cueva Merino } \\
\text { (Dos Aguas, València) }\end{array}$ & Salita interior & ca. 400 & $\mathrm{P}$ & LP & P. pardus & 2 & 1 & Sarrión, 1984; Fernández, 2004 \\
\hline 57 & $\begin{array}{l}\text { Cova de les Meravelles } \\
\text { (Alzira, València) }\end{array}$ & - & 60 & $?$ & $\mathrm{P}$ & P. pardus & 1 & 1 & This study \\
\hline 58 & $\begin{array}{l}\text { Cova del Bolomor } \\
\text { (Tavernes de Valldigna, } \\
\text { València) }\end{array}$ & XIII & 90 & A & MP (MIS 7) & P. pardus & 3 & 1 & $\begin{array}{l}\text { This study; Fernández, } \\
\text { personal communication }\end{array}$ \\
\hline 58 & $\begin{array}{l}\text { Cova del Bolomor } \\
\text { (Tavernes de Valldigna, } \\
\text { València) }\end{array}$ & EI sub XIII & & A & LP (MIS 5e) & P. pardus & 1 & 1 & $\begin{array}{l}\text { Fernández, 2004; Fernández, } \\
\text { personal communication; } \\
\text { this study }\end{array}$ \\
\hline 59 & $\begin{array}{l}\text { Cova Malladetes } \\
\text { (Barx, València) }\end{array}$ & $\begin{array}{l}\text { SE c16-17, } \\
\text { SD c22, SI c28 }\end{array}$ & 631 & A & LP (Au.) & P. pardus & 4 & 2 & This study \\
\hline 60 & $\begin{array}{l}\text { Cova Negra } \\
\text { (Xàtiva, València) }\end{array}$ & - & 100 & A & $\mathrm{P}$ & Felix pardus & 2 & 1 & Royo, 1942 \\
\hline 60 & $\begin{array}{l}\text { Cova Negra (Xàtiva, } \\
\text { València) CN1 }\end{array}$ & $\begin{array}{l}\text { S/N, SE c4, c24, } \\
\text { SF c7, SB c29, c5 }\end{array}$ & & A & $\mathrm{P}$ & P. pardus & 10 & 2 & Pérez, 1977 \\
\hline 60 & $\begin{array}{l}\text { Cova Negra (Xàtiva, } \\
\text { València) CN2 }\end{array}$ & VI, IIIa, II & & A & $\mathrm{P}$ & P. pardus & 6 & 1 & Martínez, 1996, 2009 \\
\hline 60 & $\begin{array}{l}\text { Cova Negra (Xàtiva, } \\
\text { València) CN3 }\end{array}$ & Messed level & & A & $\mathrm{P}$ & P. pardus & 3 & 1 & This study \\
\hline 61 & $\begin{array}{l}\text { Cova de Racó del Duc I } \\
\text { (Vilallonga, València) }\end{array}$ & $\begin{array}{l}\text { Infra-Solutrian } \\
\text { level }\end{array}$ & ca. 200 & A & LP (UPal) & P. pardus & 41 & 2 & This study \\
\hline 61 & $\begin{array}{l}\text { Cova de Racó del Duc II } \\
\text { (Vilallonga, València) }\end{array}$ & Chasm bottom & & $\mathrm{P}$ & $\mathrm{P}$ & P. pardus & 47 & 1 & This study \\
\hline 62 & $\begin{array}{l}\text { Cova del Corb } \\
\text { (Ondara, Alacant) }\end{array}$ & $\begin{array}{l}\text { External breccia; } \\
\text { IV }\end{array}$ & 200 & A & MP (MIS 7) & P. pardus & 1 & 1 & $\begin{array}{l}\text { Sarrión, 1990; Fernández, } \\
\text { personal communication }\end{array}$ \\
\hline 62 & $\begin{array}{l}\text { Cova del Corb } \\
\text { (Ondara, Alacant) }\end{array}$ & Internal breccia & & A & LP (UPal) & P. pardus & 1 & 1 & $\begin{array}{l}\text { Sarrión, 1990; Fernández, } \\
\text { personal communication }\end{array}$ \\
\hline 63 & $\begin{array}{l}\text { Cova Foradada } \\
\text { (Xàbia, Alacant) }\end{array}$ & VII, VI, V & 40 & A & $\operatorname{LP}(\mathrm{Au})$ & P. pardus & 7 & 3 & Pantoja et al., 2011 \\
\hline 64 & $\begin{array}{l}\text { Cova de les Calaveres } \\
\text { (Benidoleig, Alacant) }\end{array}$ & - & 131 & A & LP (UPal) & P. pardus & 1 & 1 & Aparicio et al., 1982 \\
\hline 65 & $\begin{array}{l}\text { Cova del Parat } \\
\text { (Tollos, Alacant) }\end{array}$ & - & ca. 776 & A & ELP & P. pardus & 3 & 1 & $\begin{array}{l}\text { Martínez, personal } \\
\text { communication }\end{array}$ \\
\hline 66 & El Salt (Alcoi, Alacant) & $\begin{array}{l}\text { D6, X La, Lev } \\
6 \mathrm{n}^{\circ} 3(2006)\end{array}$ & 700 & A & LP (Ms.) & P. pardus & 1 & 1 & $\begin{array}{l}\text { Morales, Brugal and Pérez, } \\
\text { personal communication; } \\
\text { this study }\end{array}$ \\
\hline 67 & $\begin{array}{l}\text { Abric del Pastor } \\
\text { (Alcoi, Alacant) }\end{array}$ & $\begin{array}{l}\text { A. clean profiles, } \\
\text { AP-1633 (2005) }\end{array}$ & 800 & A & LP (Ms.) & P. pardus & 1 & 1 & $\begin{array}{l}\text { Morales and Pérez, personal } \\
\text { communication; this study }\end{array}$ \\
\hline 68 & $\begin{array}{l}\text { Avenc de Joan Guitón } \\
\text { (Fontanars dels Alforins, } \\
\text { València) }\end{array}$ & Leopard gallery & 774 & $\mathrm{P}$ & $\mathrm{P}$ & P. pardus & 221 & 1 & This study \\
\hline 69 & $\begin{array}{l}\text { Sima de las Palomas } \\
\text { (Torre Pacheco, Murcia) }\end{array}$ & - & 75 & A & LP (Ms.) & P. pardus & 1 & 1 & $\begin{array}{l}\text { Walker, 2001; } \\
\text { Walker et al., } 2012\end{array}$ \\
\hline 70 & $\begin{array}{l}\text { Cueva de Carigüela } \\
\text { (Darro, Granada) }\end{array}$ & - & 1020 & A & LP (Ms.) & P. pardus & 3 & 1 & Bouchud, 1969 \\
\hline 71 & $\begin{array}{l}\text { Boquete de Zafarraya } \\
\text { (Alcaucín, Málaga) }\end{array}$ & A, B, C, D, E, F, G & ca. 1000 & A & LP (Ms.) & P. pardus & 247 & 18 & Barroso et al., 2006 \\
\hline 72 & Devil's Tower (Gibraltar) & - & $<10$ & A & LP (Ms.) & P. pardus & \multicolumn{2}{|l|}{ Presence } & Currant, 2000 \\
\hline 73 & Genetista Cave (Gibraltar) & - & $<10$ & A & LP (Ms.) & P. pardus & \multicolumn{2}{|c|}{ Presence } & Currant, 2000 \\
\hline 74 & Vanguard Cave (Gibraltar) & C-D units & $<5$ & A & LP (Ms.) & P. pardus & 7 & - & Stringer et al., 2008 \\
\hline 75 & Gorham's Cave (Gibraltar) & IV & $<5$ & A & LP (Ms.) & P. pardus & 4 & - & $\begin{array}{l}\text { Currant, 2000; } \\
\text { Rodríguez et al., } 2010\end{array}$ \\
\hline 75 & Gorham's Cave (Gibraltar) & III & & A & LP (So.) & P. pardus & 2 & - & $\begin{array}{l}\text { Currant, 2000; } \\
\text { Carrión et al., 2008; } \\
\text { Rodríguez et al., } 2010\end{array}$ \\
\hline 76 & $\begin{array}{l}\text { Gruta do Escoural } \\
\text { (Montemor-o-Novo) }\end{array}$ & - & 350 & A & $\mathrm{P}$ & P. pardus & 19 & 1 & Cardoso, 1993, 1996 \\
\hline 77 & $\begin{array}{l}\text { Gruta da Figueira } \\
\text { Brava (Setúbal) }\end{array}$ & C. 2 & $<5$ & A & LP (Ms., 30,9 ka) & P. pardus & 3 & 1 & Cardoso, 1993, 1996 \\
\hline 78 & Pego do Diablo (Loures) & 2 & 250 & A & LP (Au.) & cf. P. pardus & 1 & 1 & $\begin{array}{l}\text { Zilhão et al., 2010a; } \\
\text { Valente, 2004a, b }\end{array}$ \\
\hline 79 & $\begin{array}{l}\text { Pedreira das Salemas } \\
\text { (Loures) }\end{array}$ & C. 2 & 240 & A & LP (Au., 29,8 ka) & P. pardus & 1 & 1 & Antunes et al., 1989 \\
\hline 80 & $\begin{array}{l}\text { Gruta das Fontaínhas } \\
\text { (Cadaval) }\end{array}$ & - & 400 & A & LP (So., 22,7 ka) & P. pardus & 2 & 1 & Cardoso, 1993, 1996 \\
\hline 81 & Furninha (Peniche) & $\begin{array}{l}4-4.5 ; 7-7.5 \\
8.8-9.3\end{array}$ & $<5$ & A & LP (Ms.) & P. pardus & 4 & 1 & $\begin{array}{l}\text { Cardoso, 1993, 1996; } \\
\text { Brugal et al., } 2012\end{array}$ \\
\hline
\end{tabular}


Table 13 (continued)

\begin{tabular}{|c|c|c|c|c|c|c|c|c|c|}
\hline $\mathrm{N}^{\circ}$ & Site & Level & masl & Context & Chronology & Taxon & NISP & MNI & References \\
\hline 82 & Casa da Moura (Óbidos) & - & 160 & A & LP (Au., 25 ka) & P. pardus & 1 & 1 & Valente, 2004a \\
\hline 83 & $\begin{array}{l}\text { Algar da Manga Larga } \\
\text { (Porto de Mós) }\end{array}$ & - & 460 & $\mathrm{P}$ & LP & P. pardus & ca. 10 & 1 & Cardoso and Regala, 2006 \\
\hline 84 & $\begin{array}{l}\text { Gruta da Oliveira } \\
\text { (Torres Novas) }\end{array}$ & 13 & ca. 115 & A & LP (Ms.) & Panthera sp. & 1 & 1 & Zilhão et al., 2010b \\
\hline 85 & Gruta do Caldeirao (Tomar) & Ms. level & 120 & A & LP (Ms.) & P. pardus & 1 & 1 & Davis, 2002 \\
\hline 85 & Gruta do Caldeirao (Tomar) & So. level & & A & LP (So.) & P. pardus & 6 & 1 & Davis, 2002; Cardoso, 1996 \\
\hline 86 & Lorga de Dine (Vinhais) & - & 787 & A & LP (Ms.) & P. pardus & 2 & 1 & Cardoso, 1993, 1996 \\
\hline
\end{tabular}

than that of the African leopard. This feature also appears in other crania found in the Iberian Peninsula such as Abric Romaní, Algar da Manga Larga and Allekoaitze (Cáceres et al., 1993; Cardoso and Regala, 2006; Corral, 2012; Altuna and Mariezkurrena, 2013), which for some authors may be typical of female leopards (Ghezzo and Rook, 2015). In the present-day African leopard this area is more elongated and does not project backwards (Sims, 2012). Likewise, the zygomatic bones of AJG do not open up laterally as much as in the African species and they run more parallel to the neurocranium (Fig. 8A, B). The maxilla presents a very hypsodont P4 (Fig. 8C, E).

The temporal sutures join further back from the post-orbital process, the sagittal crest shows little development, the braincase is short and wide and there is no clear post-orbital constriction (Fig. 8A, C), which are typical features of females (Ghezzo and Rook, 2015). The relatively small size of the cranium appears to be the consequence of sexual dimorphism (Christiansen and Harris, 2012) and confirms that it is a female, like the one found at Algar da Manga Larga (Cardoso and Regala, 2006).

The cranium is not as long as that of African females, a feature that it shares with P. uncia. In contrast, AJG has a greater frontal and nasal width than that of the African leopard (Fig. 8A, D), a feature which is typical of P. pardus spelaea and P. uncia (Sims, 2012;
Diedrich, 2013) and which can be interpreted as an adaptation to mountainous areas (Diedrich, 2013). This greater nasal width would allow them to heat the air in cold atmospheres (Hemmer, 1972).

As regards the cranial dimensions of AJG, the total length is within the range of female $P$. pardus and is almost the minimum for males. Compared with other fossil material (Table 2), AJG is longer than Algar da Manga Larga, but shorter than Allekoaitze and Aintzulo, and very similar to the smallest female from Equi. Present-day P. pardus specimens show a high degree of biometric variability according to sexual dimorphism, with males generally being larger than females, although there may be overlaps. This variability is also seen in the fossil material. AJG is close to the maximum for $P$. uncia, greater than the maximum for female Puma and similar to the minimum for males.

The condylobasal length of AJG is shorter than the minimum for male $P$. pardus and is within the upper range for females. In the case of felines, this cranial measurement appears to be strongly related to the overall size of the animal (Turner and O'Regan, 2002). AJG is clearly larger than Algar da Manga Larga but smaller than Aintzulo and Allekoaitze. AJG is larger than the maximum for $P$. uncia, it is within the range of male and larger than the maximum for female Puma.

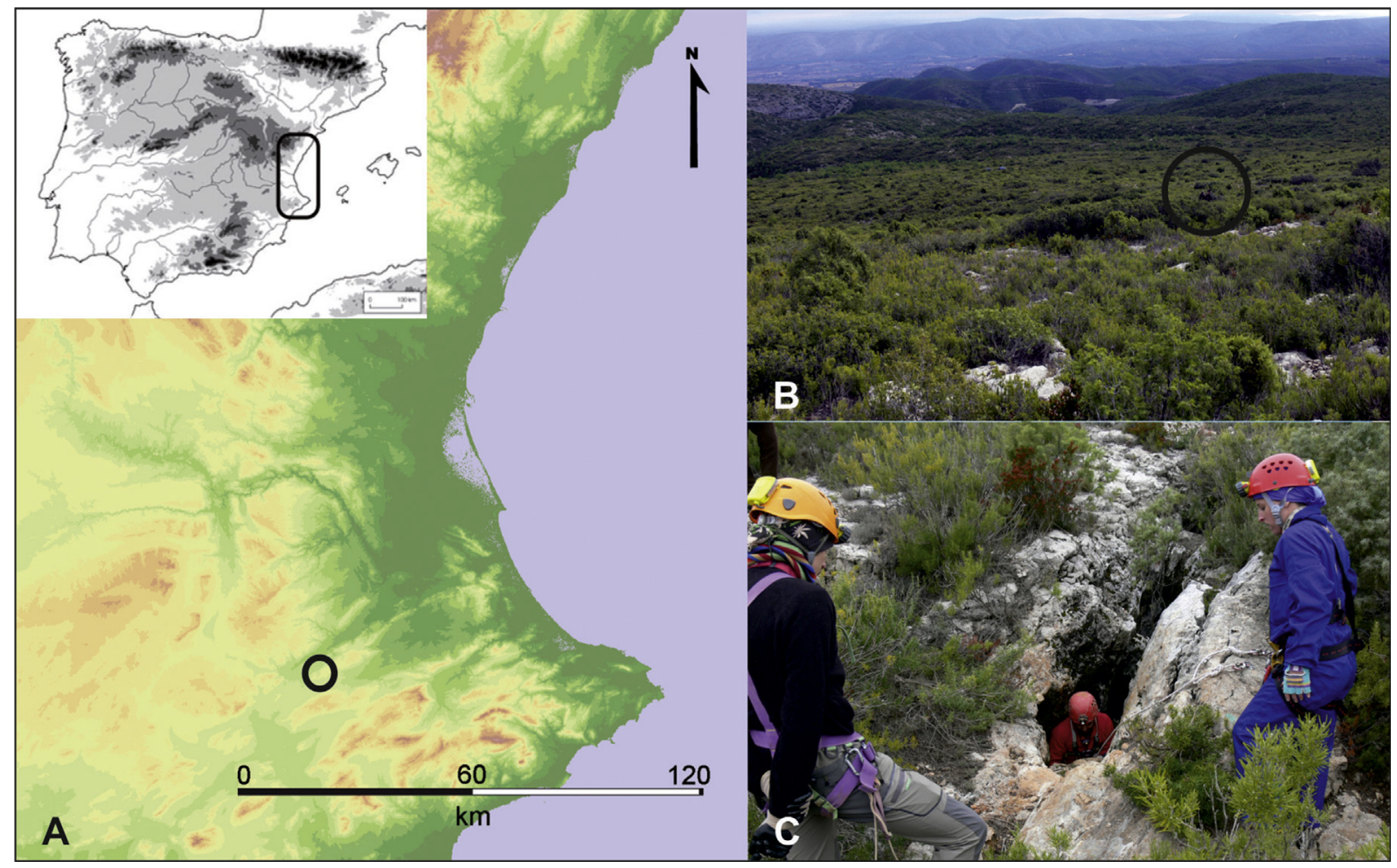

Fig. 1. A) Geographical location of Avenc de Joan Guitón chasm; B) Immediate environment; C) View of the entry to the chasm. 


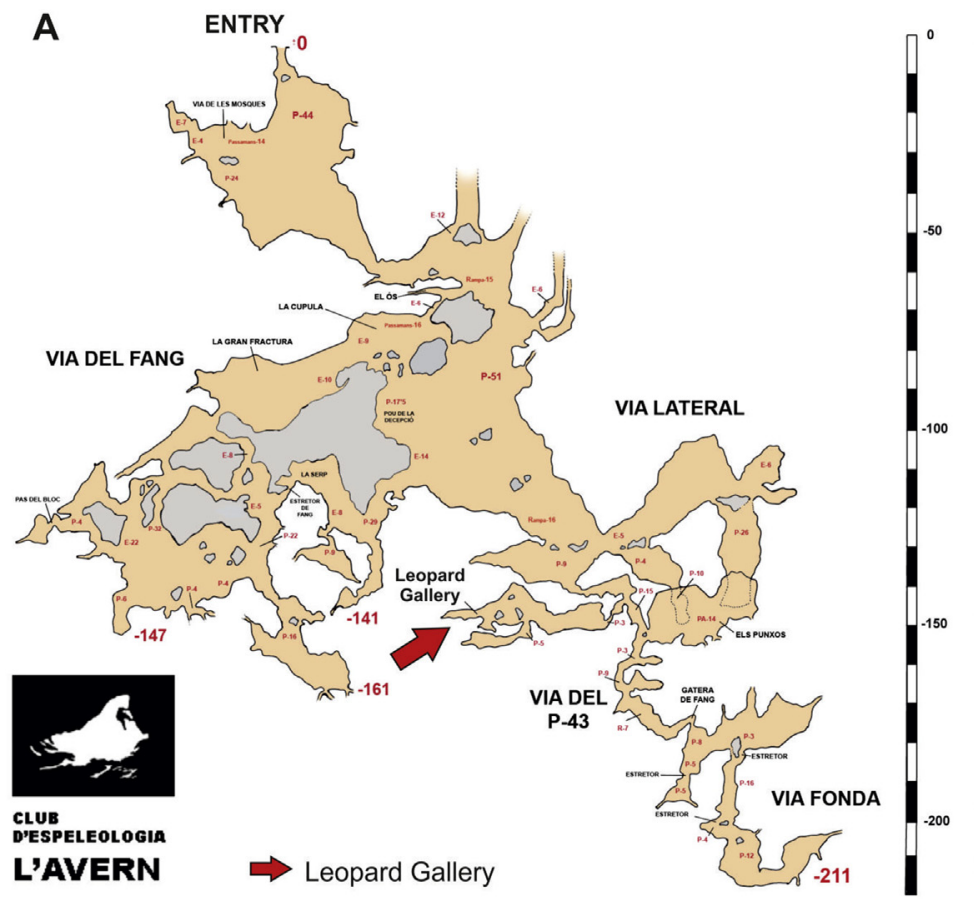

B

Development: $1.423 \mathrm{mts}$

Extension: $858 \mathrm{mts}$

Topography and Drawing

Vicent Sanchis and Rebeca Diaz

Exploration

Vicent Sanchis and Rebeca Díaz

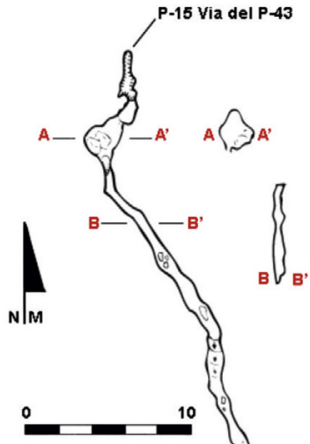

Scale 1:200
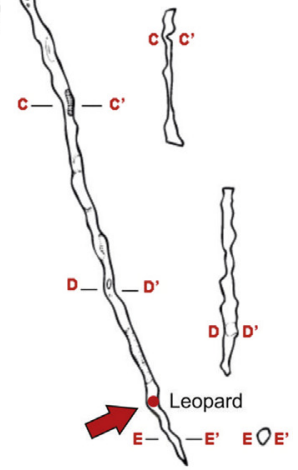

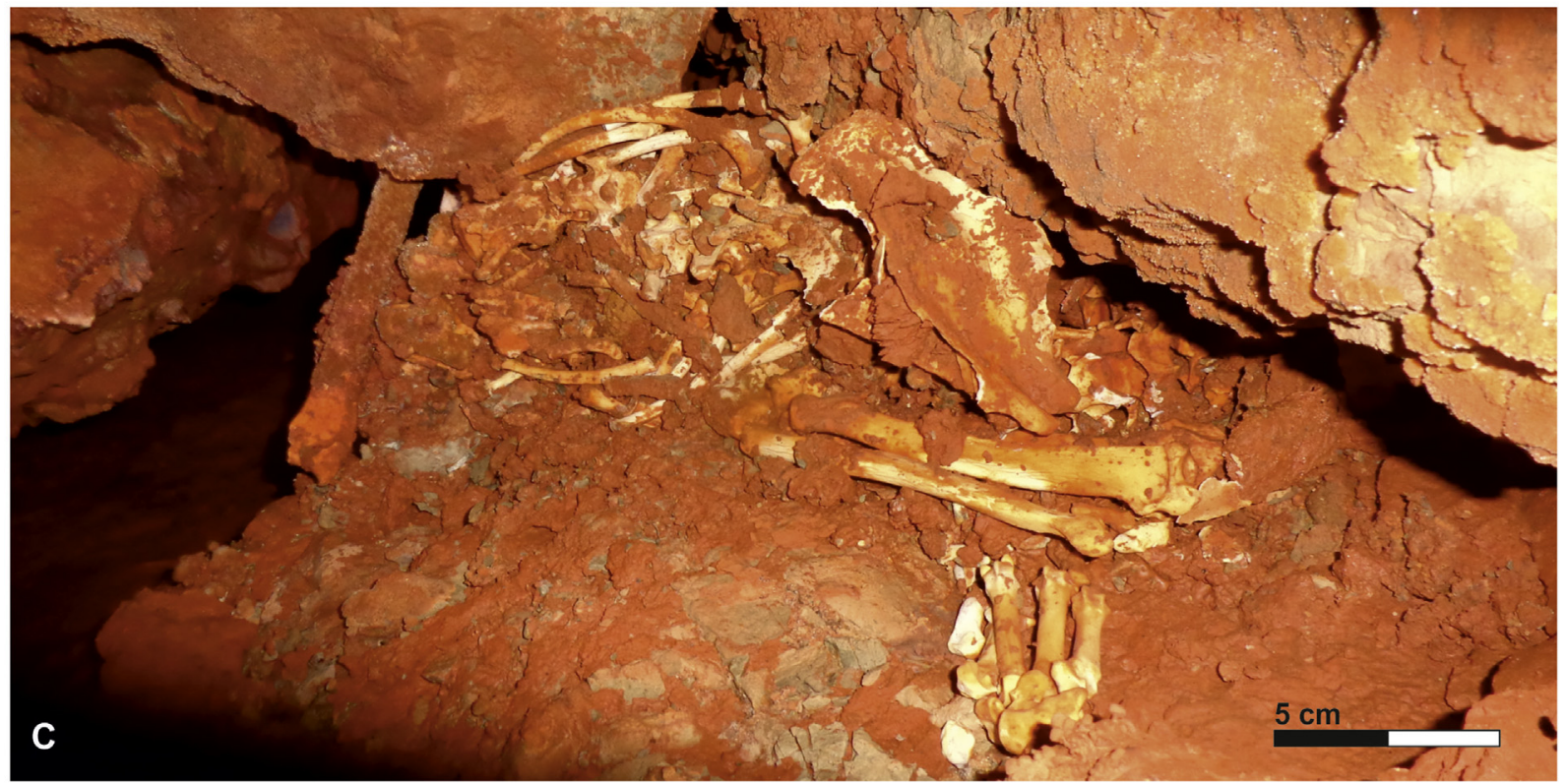

Fig. 2. A) Chasm topography section (Avenc de Joan Guitón); B) Leopard Gallery plant; C) Leopard remains before their recovery.

The zygomatic width is similar to Algar da Manga Larga. This value is within the lower range of male and upper range of female $P$. pardus, close to the maximum for $P$. uncia and greater than the maximum for female Puma.

The minimum distance between the orbits is within the range of female and male $P$. pardus. This measurement is similar to that of Algar da Manga Larga, equal to the minimum for P. uncia and greater than the maximum for Puma.

The post-orbital constriction width is very similar to Algar da Manga Larga, Allekoaitze and Aintzulo, greater than the maximum for female $P$. pardus and within the range of males. This value in AJG is smaller than the minimum for $P$. uncia and greater than that of female and within the range of male Puma.
The cranial lengths situate the AJG leopard within the range of female $P$. pardus, which is consistent with the crania of female fossils (Algar da Manga Larga and the smallest individual from Equi) (Fig. 9). The crania from Allekoaitze and Aintzulo present dimensions that are more consistent with males, although recently the first of them has been classified as a female based on its morphology (Ghezzo and Rook, 2015).

AJG has a short, wide cranium typical of female $P$. pardus fossils from southern areas of Europe. If we compare AJG with fossils such as Algar da Manga Larga, the zygomatic width is similar. The same applies to the minimum width of the orbits or the neurocranial width. Post-orbital constriction in AJG is similar to fossil values and greater than for female P. pardus. The frontal width of Pleistocene 


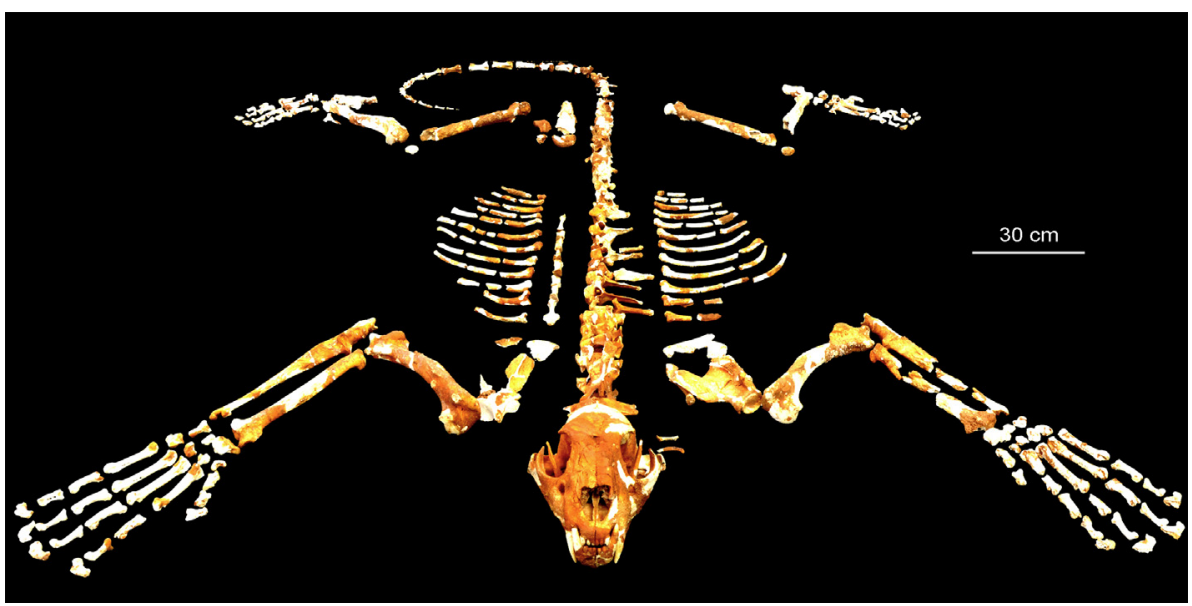

Fig. 3. Leopard skeleton from Avenc de Joan Guitón (No. 30464). The white bits are due to differential preservation.

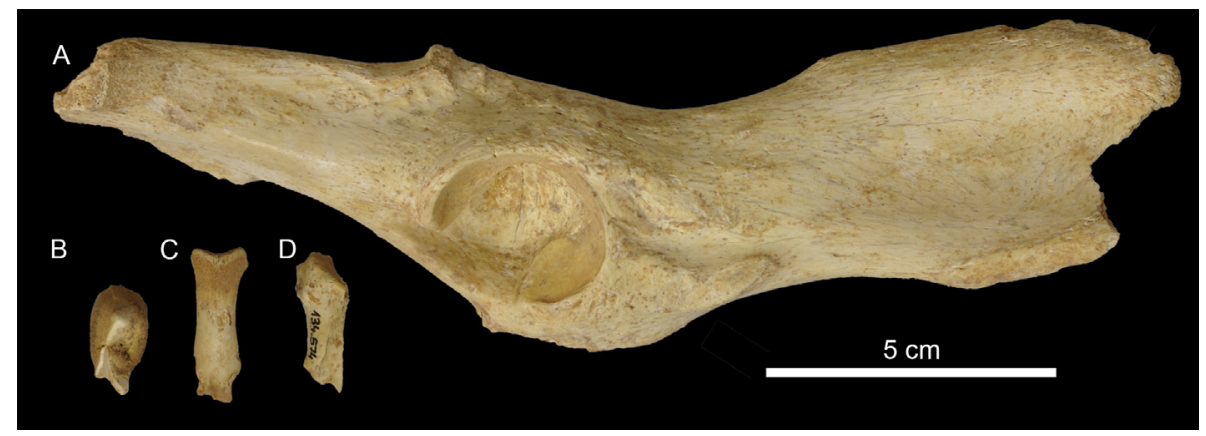

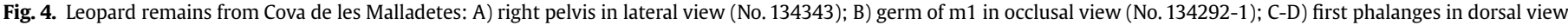
(No. 134292-2; 134574).

crania is greater than that of female P. pardus.

If we consider the idea put forward by Diedrich (2013) and Sauqué et al. (2014a), the AJG cranium has morphometric features that lie somewhere between $P$. uncia and present-day African leopards, which would be typical of the European subspecies $P$. pardus spelaea. For Ghezzo and Rook (2015) the features of the AJG cranium could be explained by the intraspecific variability and sexual dimorphism that exist in $P$. pardus. Considering that the sample of leopards from the Iberian Peninsula does not provide a large enough number of crania from both the Middle and Late Pleistocene with morphological or metric differences that demonstrate the existence of a subspecies, we maintain the specific attribution for the AJG specimen.

\subsubsection{Mandible}

The AJG mandibles have two mental foramina in the buccal view, the mesial in the middle of the diastema and the distal under p3 (Fig. 8C). A similar number is observed in other mandibles found in the Mediterranean area (Abric Romaní and Boquete de Zafarraya), whereas those found in the rest of the peninsula show three (Algar da Manga Larga, Allekoaitze, Torrejones and Los Rincones). Some authors have used this criterion to distinguish P. pardus from P. uncia (three foramina) (Madurell-Malapeira et al., 2010), although others consider that this characteristic is part of intraspecific variability and therefore lacks systematic value (Cardoso and Regala, 2006; Testu et al., 2011; Sauqué and Cuenca-Bescós, 2013), as Iberian examples seem to confirm.
The angle formed by the basal and anterior border of the ramus is obtuse $\left(>90^{\circ}\right)$ and shows a receding symphysis that is characteristic of $P$. pardus (Fig. 8C, E), whereas in P. uncia it is almost at a right angle and the symphysis is semivertical (Testu, 2006; Christiansen, 2008; Testu et al., 2011). Some of the Iberian leopards present the morphology described for P. pardus (Abric Romaní, the large specimen from Zafarraya, Allekoaitze and AJG); however, others are more similar to that of P. uncia (Algar da Manga Larga, Los Rincones and the small specimen from Zafarraya) (Cáceres et al., 1993; Barroso et al., 2006; Cardoso and Regala, 2006; Altuna and Mariezkurrena, 2013; Sauqué and Cuenca-Bescós, 2013).

The basal profile of the horizontal ramus is convex at the level of $\mathrm{m} 1$ (Fig. 8C, E), which is typical of the present-day and Late Pleistocene (Los Rincones) P. pardus, whereas $P$. uncia has a straight profile (Hemmer, 1972; Testu, 2006). This may also be a nondefining feature, as a European leopard fossil specimen (Caune de l'Arago) appears without this convexity (Testu, 2006).

The masseteric fossa is deep and reaches the level of the protoconid of $\mathrm{m} 1$. This feature is observed in various European Pleistocene leopards (Testu, 2006; Testu et al., 2011; Sauqué and Cuenca-Bescós, 2013), although it also appears in Puma concolor and P. uncia (Madurell-Malapeira et al., 2010).

The coronoid process is quite vertical, projecting backwards less than in the present-day P. pardus (Testu, 2006). Likewise, the condyle is positioned somewhat further back than the posterior border of the coronoid process (Fig. 8C).

The mandible is higher behind $\mathrm{m} 1$ than in front of $\mathrm{p} 3$ (Table 3), 


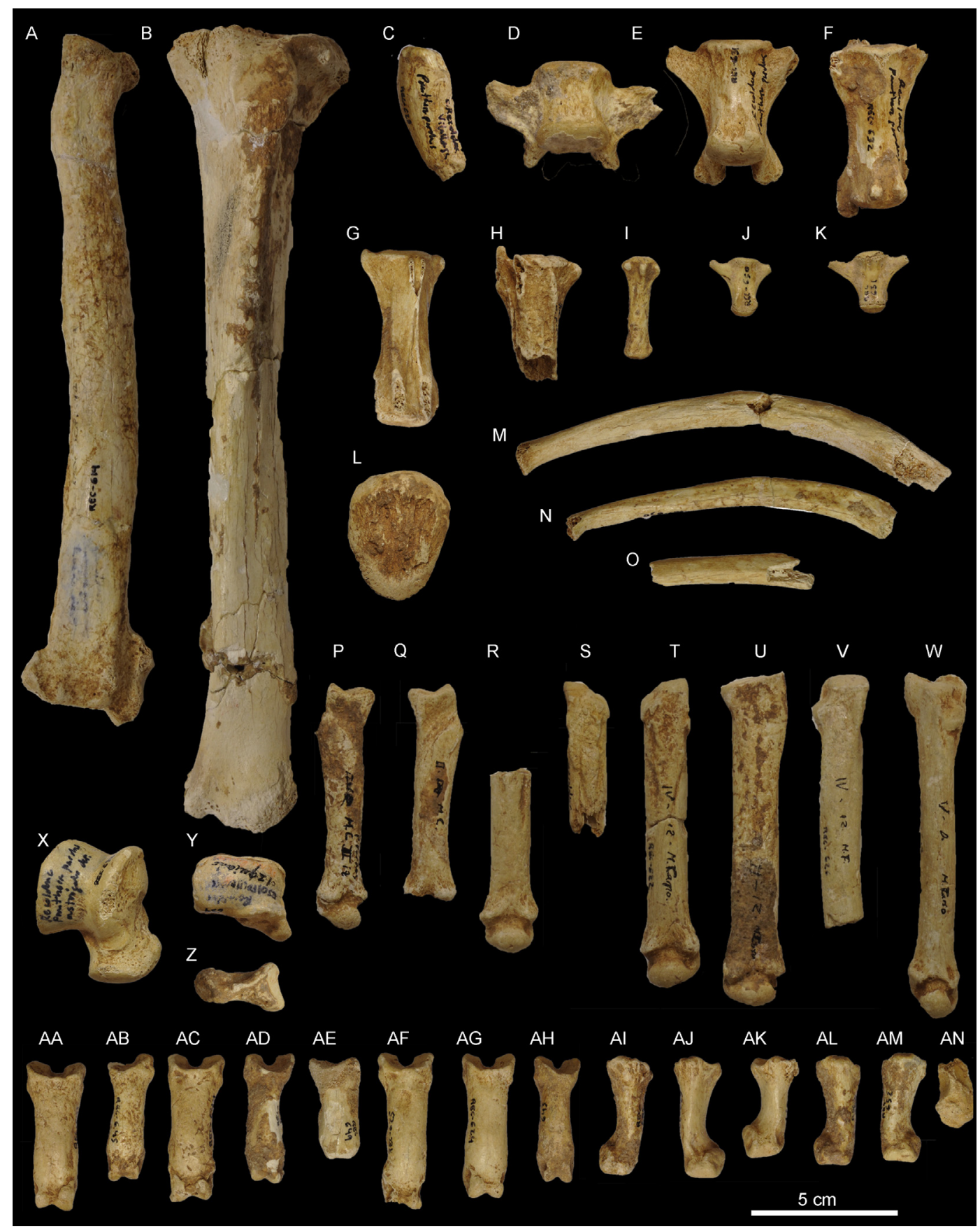

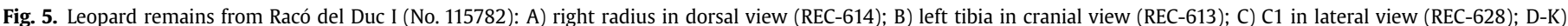

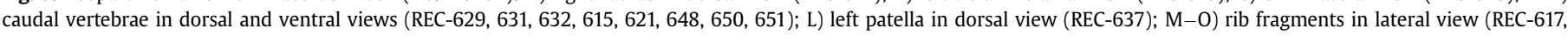

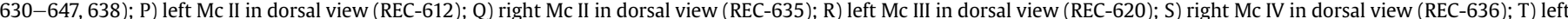

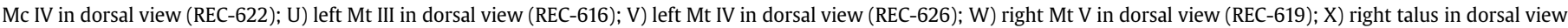

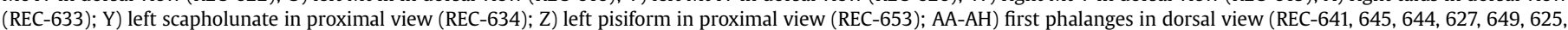
624, 643); AI-AM) second phalanges in dorsal view (REC-642, 623, 639, 640, 652); AN) third phalanx in lateral view (REC-655).

which is observed in all the Iberian P. pardus fossils except that of Algar da Manga Larga (Sauqué and Cuenca-Bescós, 2013).

As regards the dimensions of the AJG mandible (Table 3), the total length is very similar to that of Abric Romaní, Los Rincones and Caune de l'Arago (Cáceres et al., 1993; Testu, 2006; Sauqué and Cuenca-Bescós, 2013) and greater than that of Algar da Manga Larga (Cardoso and Regala, 2006). This measurement in the AJG specimen is close to average for present-day leopards and greater than for P. uncia and Puma.
The diastema index (length of the diastema relative to that of p3) (Hemmer, 1971) is long (120.5), with values close to those of Karaïn E (122.3), Observatoire (118.4) and present-day P. pardus (121), while it differs considerably from P. uncia (89.7) and Puma pardoides of Stránská Skála (62.5), which have a short diastema. But some Late Pleistocene $P$. pardus specimens also have a short diastema, such as those from Los Rincones, Algar da Manga Larga, Zafarraya and Triagalnata (Spassov and Raychev, 1997; Barroso et al., 2006; Cardoso and Regala, 2006; Testu, 2006; Testu et al., 


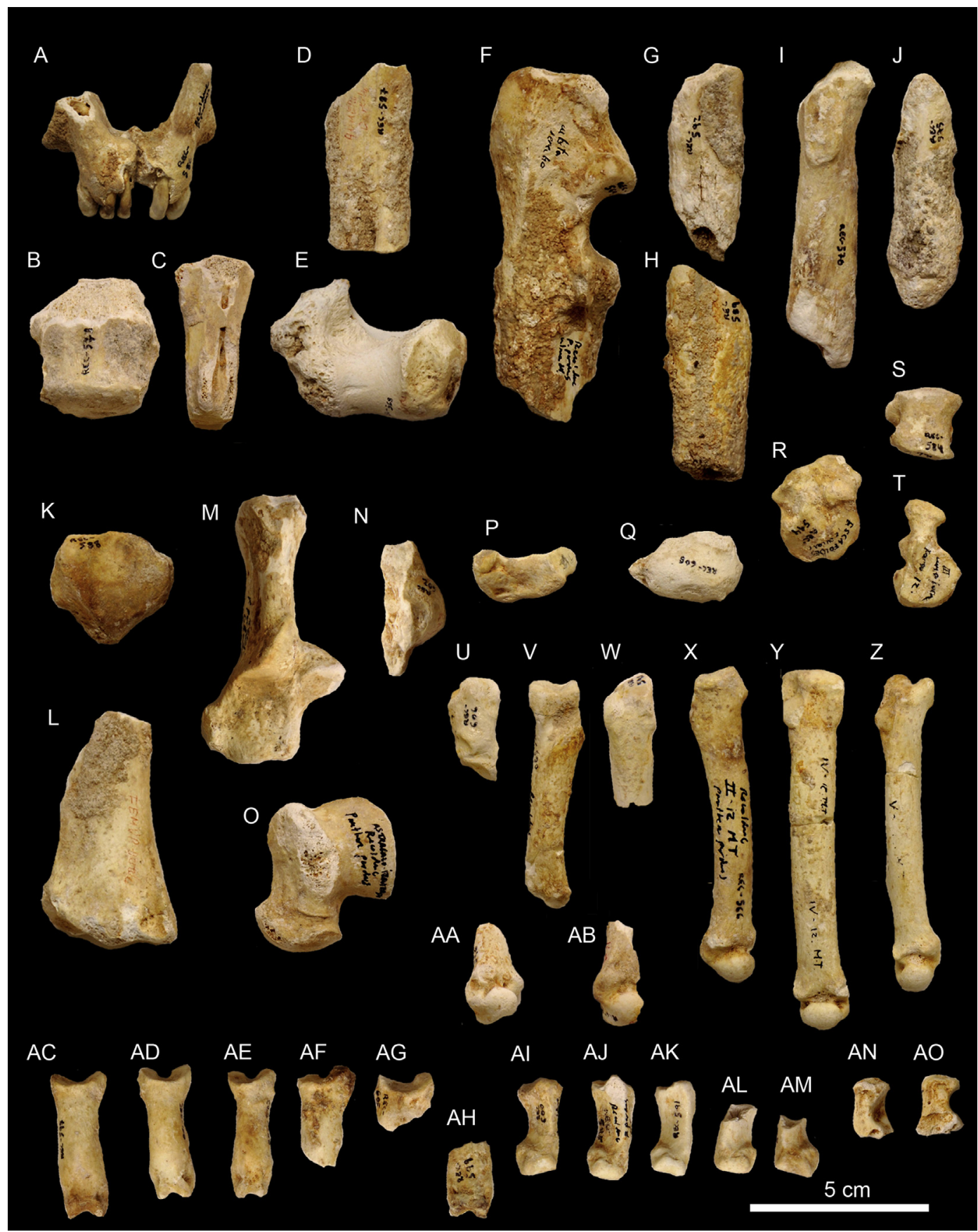

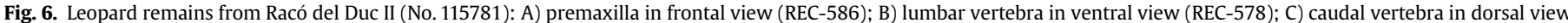

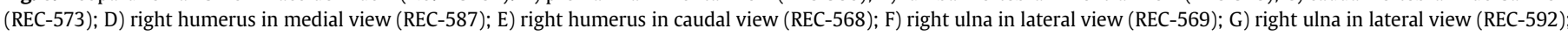

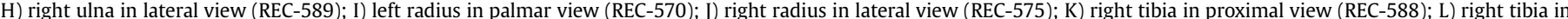

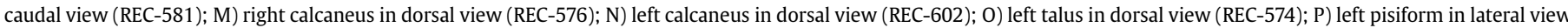

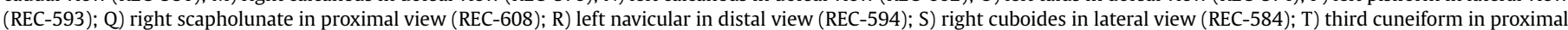

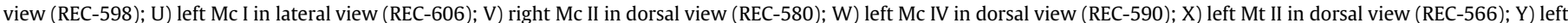

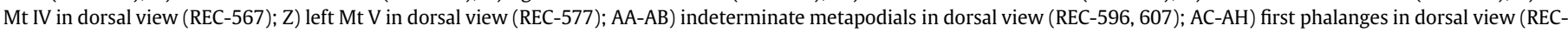
$597,585,583,603,605,599)$; AI-AM) second phalanges in dorsal view (REC-600, 595, 591, 612, 610); AN-AO) third phalanges in lateral view (REC-611, 604).

2011; Sauqué and Cuenca-Bescós, 2013).

The mandibular robustness (height of the mandibular ramus behind $m 1$ relative to the length of $m 1$ ) (155.3) is similar to that of various Late Pleistocene specimens such as those of Abric Romaní (156.4), Algar da Manga Larga (149,3), Torrejones (158.2) and Triagalnata (154.6) (data compiled by Sauqué and Cuenca-Bescós, 2013), differing from the present-day P. uncia (137.2) (Testu, 2006).
The length of the molar row of some fossils found in the Iberian Peninsula, which is very similar to the regression line of the present-day P. uncia (Fig. 10), could indicate the adaptation of both species to a similar mountain habitat with scarce prey, which they would have to exploit to the full (Spassov and Raychev, 1997; Sauqué and Cuenca-Bescós, 2013).

Despite some similarities with $P$. uncia, the other features 


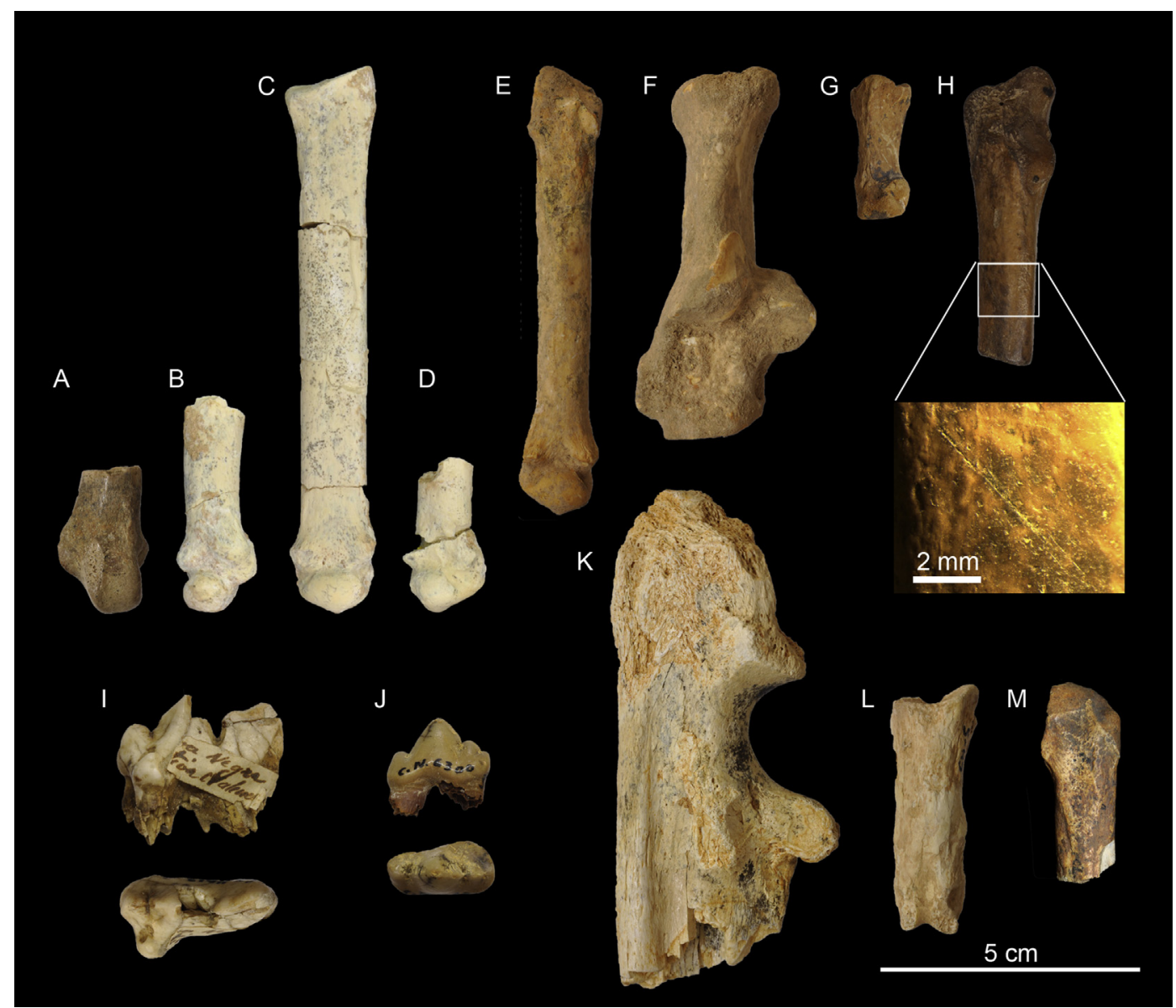

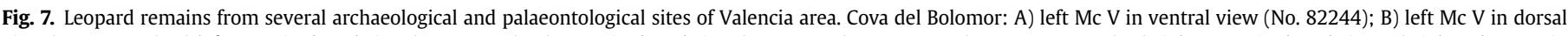

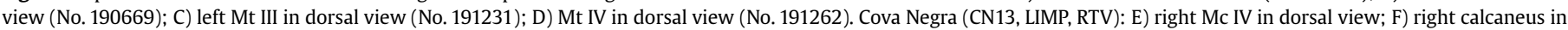

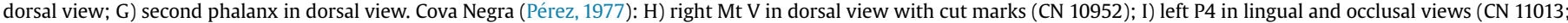

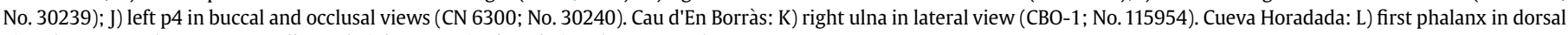
view (No. 115922). Cova Meravelles: M) right Mc IV in dorsal view (No. 116235).

confirm that the AJG mandible belongs to P. pardus.

\subsubsection{Upper teeth}

The incisors are inserted into the premaxilla (Fig. 8D), are frontally flat, have a posterior cusp and increase in size from I1 to I3, a common morphology in leopards (Table 4) (Testu, 2006). There is a short diastema between incisors and canines.

The canines have a rounded cross section and are massive and laterally flattened. The crown, which is straight, shows some backwards torsion. There is a well-defined ridge on the distal edge of the crown. On the buccal and lingual surfaces of the crown there are two prominent longitudinal grooves, a feature that rules out $P$. onca and Puma concolor (Sims, 2005, 2012) (Fig. 8C, E). These grooves are also seen in Aintzulo, Algar da Manga Larga and Caune de l'Arago (Testu, 2006; Cardoso and Regala, 2006; Altuna and Mariezkurrena, 2013). The dimensions of the canines vary somewhat between the different fossil specimens found in the Iberian Peninsula; they are within the range of present-day leopards and are larger than those of $P$. uncia (Table 4).

Both P2 are present (Fig. 8C, E) and the crown has a low cusp and two ridges, the posterior one being more prominent. A slight cingulum is visible in the distal area (Table 4).

P3 has a paracone followed by a weakly developed hypocone bordered by a cingulum (Fig. 8C). On the mesial-lingual border there is a weakly developed parastyle, which is rare in $P$. onca
(Baryshnikov, 2011). The mesial-distal length is similar to the present-day $P$. pardus, greater than that of Algar da Manga Larga and $P$. uncia, and shorter than most fossils of Iberian leopards (Table 4).

P4 has a very high crown (Fig. 8C). The parastyle is round and the paracone is slightly curved backwards and has two well-defined vertical ridges; the posterior ridge of the paracone and the anterior ridge of the metacone form an open angle. The protocone is round and well developed. The outer length is similar to that of Algar da Manga Larga and is smaller than that of most Iberian leopards (Table 4). All these features appear to be common to Late Pleistocene leopards (Diedrich, 2013).

M1 is a small oval residual tooth (mesial-distal GL: 7.3)

\subsubsection{Lower teeth}

The left i1 is displaced and has a compressed root in the buccallingual direction with some lateral torsion. It has a well closed apex. The upper third of the crown is broken. The other incisors are inserted into the mandible and increase in size from i1 to i3 (Table 5; Fig. 8D).

The lower canines are shorter than the upper ones (Fig. 8D). They have a groove on the buccal surface (Fig. 8C), although it is possible there may be two and they may also be present on the lingual surface, hidden by the occlusion (the cranium and the mandible are attached to each other). The mesial-distal length is 

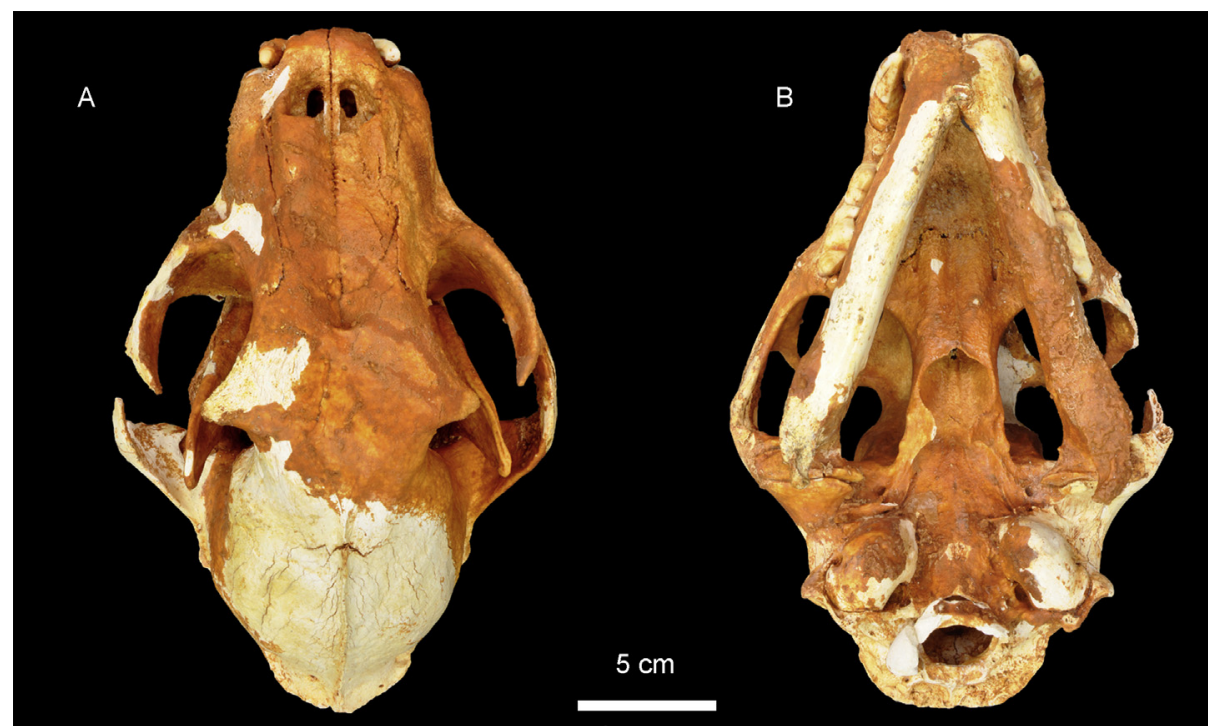

C

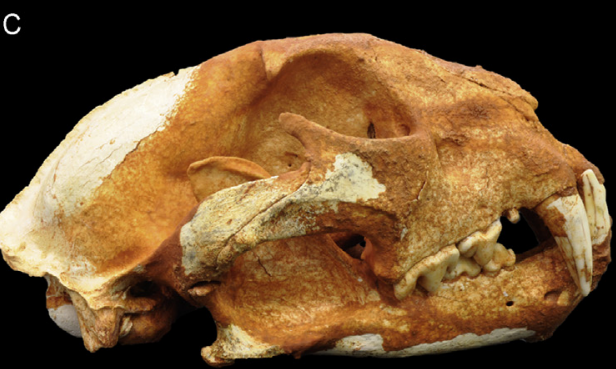

D

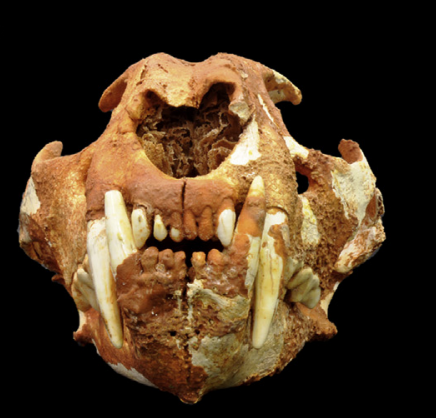

E

F

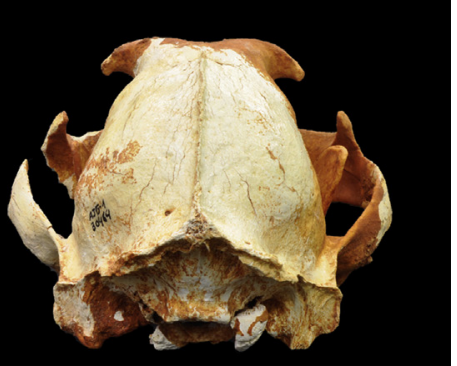

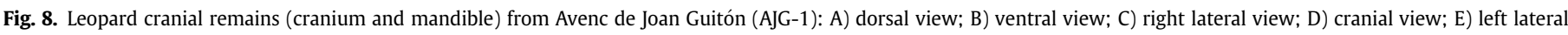
view; F) caudal view.

greater than that of most Iberian leopard fossils and also that of the present-day P. pardus and P. uncia (Table 5).

A long diastema separates the canine from p3 (Fig. 8C, D). The jugal teeth are very close together. $\mathrm{p} 3$ is trilobed and has a clearly visible circular paraconid, a feature which is seen in $P$. uncia and Puma concolor (Madurell-Malapeira et al., 2010). There is a welldeveloped protoconid and well-differentiated metaconid with a distal cingulum on the buccal surface. The tooth narrows slightly in the middle section. The dimensions of AJG coincide with the range found in Iberian fossils and are generally greater than average for present-day P. pardus and P. uncia (Table 5).

p4 is trilobed. It is difficult to describe (only seen from the lingual surface). It has a well-differentiated paraconid that slopes forward slightly. There is a well-developed, almost vertical protoconid (Table 5).

$\mathrm{m} 1$ has two main crests (paraconid and protoconid). There is no evidence of any metaconid on the distal border of the protoconid on the buccal side of the talonid, a typical feature of $P$. uncia (O'Regan and Turner, 2004). This is a discriminating criterion that confirms its attribution to P. pardus. The paraconid is less developed than the protoconid that has a small talonid, which is also typical of P. pardus (Schmid, 1940).

The ratio between the length of $m 1$ (Table 5) and that of the mandible (Table 3 ) is very similar $(0.140)$ to the average for European fossils (Algar da Manga Larga, Observatoire, Los Rincones, Abric Romaní and Zafarraya), exceeds the maximum for the present-day $P$. pardus and is within the range of $P$. uncia (Cardoso and Regala, 2006; Testu, 2006; Sauqué and Cuenca-Bescós, 2013).

\subsubsection{Forelimb}

The scapula has an oval glenoid cavity and very weakly developed coracoid process. The spine is well differentiated from the body and the acromion is very curved medially (Fig. 11A, B). There are few examples of this bone in the Iberian fossil record (Table 6). 


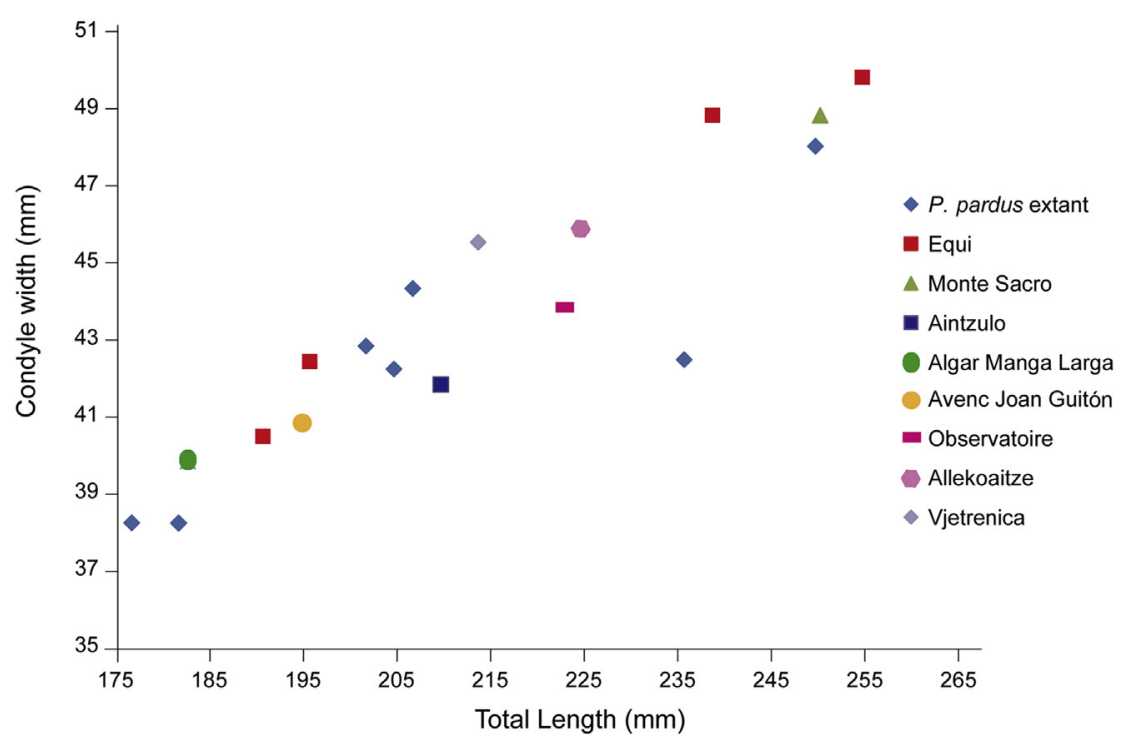

Fig. 9. Graph representing total length versus condyle width of fossil and extant leopard crania from several regions (measurements from Appendix A).

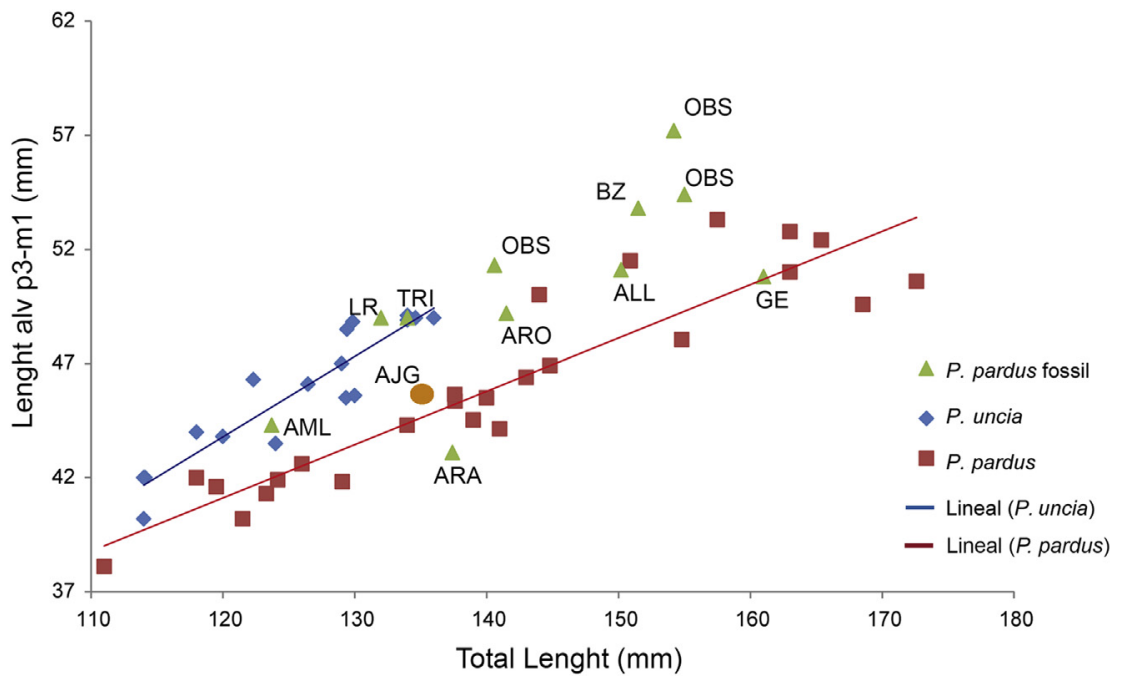

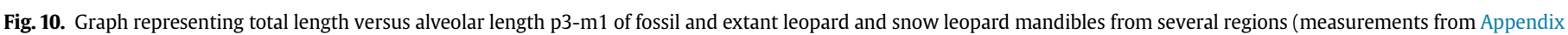
B).

As regards the proximal epiphysis (humerus), the greater tubercle is higher than the lesser tubercle, separated by a very deep intertubercular groove that runs along the medial surface (Fig. 11C). The proximal articular head projects backwards craniocaudally and shows an oval morphology in proximal view. In cranial view the diaphysis is very straight, thin and laterally flattened, with backwards torsion in the proximal third. The deltoid crest is not very prominent and is reduced to a small lateral ridge that connects with the cranial margin of the diaphysis. The distal epiphysis has a welldeveloped medial epicondyle with a long oval supracondylar foramen above it. The distal trochlea is wide with a long, slender, cylindrical capitulum. The olecranon fossa is quite deep, which is not a feature of $P$. uncia and Puma pardoides (Madurell-Malapeira et al., 2010). The radial fossa (medial side) is wide and shallow. The specimen from Aintzulo is longer and more robust than AJG. The dimensions of AJG are within the range of leopard fossils (Table 6).

The radius has an oval proximal epiphysis with some medial torsion. The bicipital tuberosity is oval, very prominent and is located on the lateral-palmar side of the proximal diaphysis, below the joint. There is backwards torsion in the distal part of the bone. The diaphysis has a semicircular cross section and is dorsopalmarly flattened. The distal extremity is well developed and is wider than the diaphysis. There is a very pronounced styloid process that is pointed and projects vertically. The distal articular surface is subrectangular with a very prominent transverse ridge. The dimensions of the radius are within the range of Iberian fossils (Table 6; Fig. 11D).

The olecranon process of the ulna is highly developed and is forked. The proximal caudal edge is rounded and presents medial torsion. The lower border of the trochlear notch has a distally oriented facet. Below the radial notch the diaphysis is flat or even slightly convex. The diaphysis, which is very straight and has a triangular cross section, is flattened laterally, narrowing in a proximal-distal direction. The distal extremity, which is also flattened, has an oval cross section. It has a subcircular styloid process, 


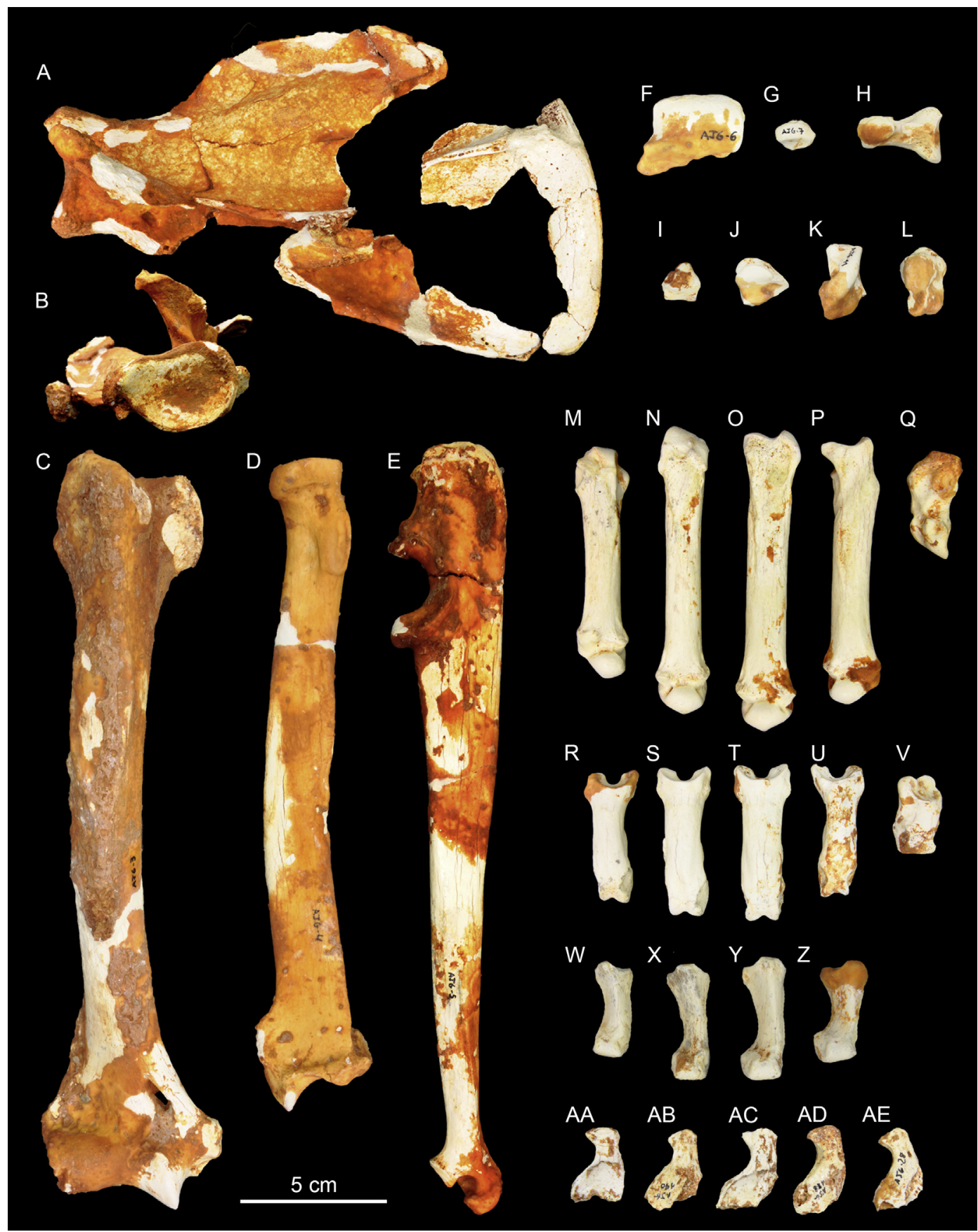

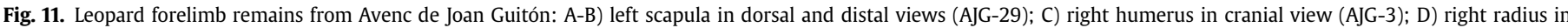

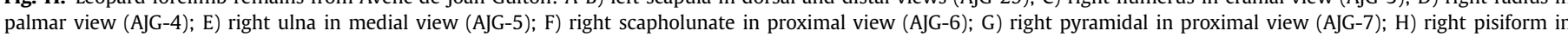

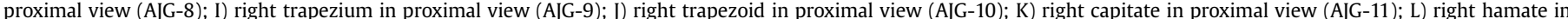

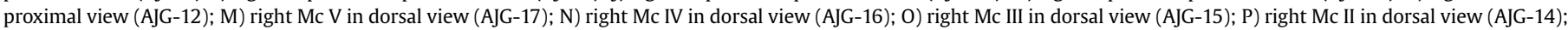

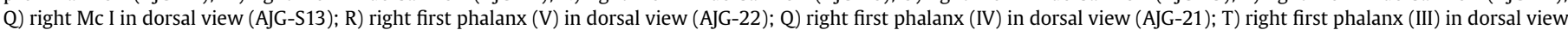

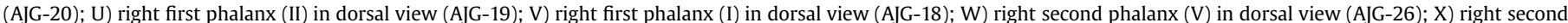

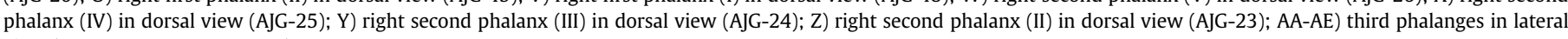
view (AJG-28, 189, 188, 190, 191).

with a rounded articular circumference that projects dorsally. The distal crest is pronounced on the dorsal side. The dimensions are smaller than those of Los Rincones (Table 6; Fig. 11E).

There are very few carpal bones in the Iberian fossil record (Table 6).

The scapholunate is subrectangular and mediolaterally elongated. It has a very robust posterior-medial process. In the proximal side, it has a convex facet that articulates with the radius (Fig. 11F). In distal view, the surface is divided into four facets at an oblique angle that articulate with the carpal bones of the distal segment.

The pyramidal is small and oval and articulates with the scapholunate, pisiform and hamate. In proximal view, the surface forms an obtuse angle, divided lateromedially by a facet, and the articulation with the pisiform is situated on the palmar margin 


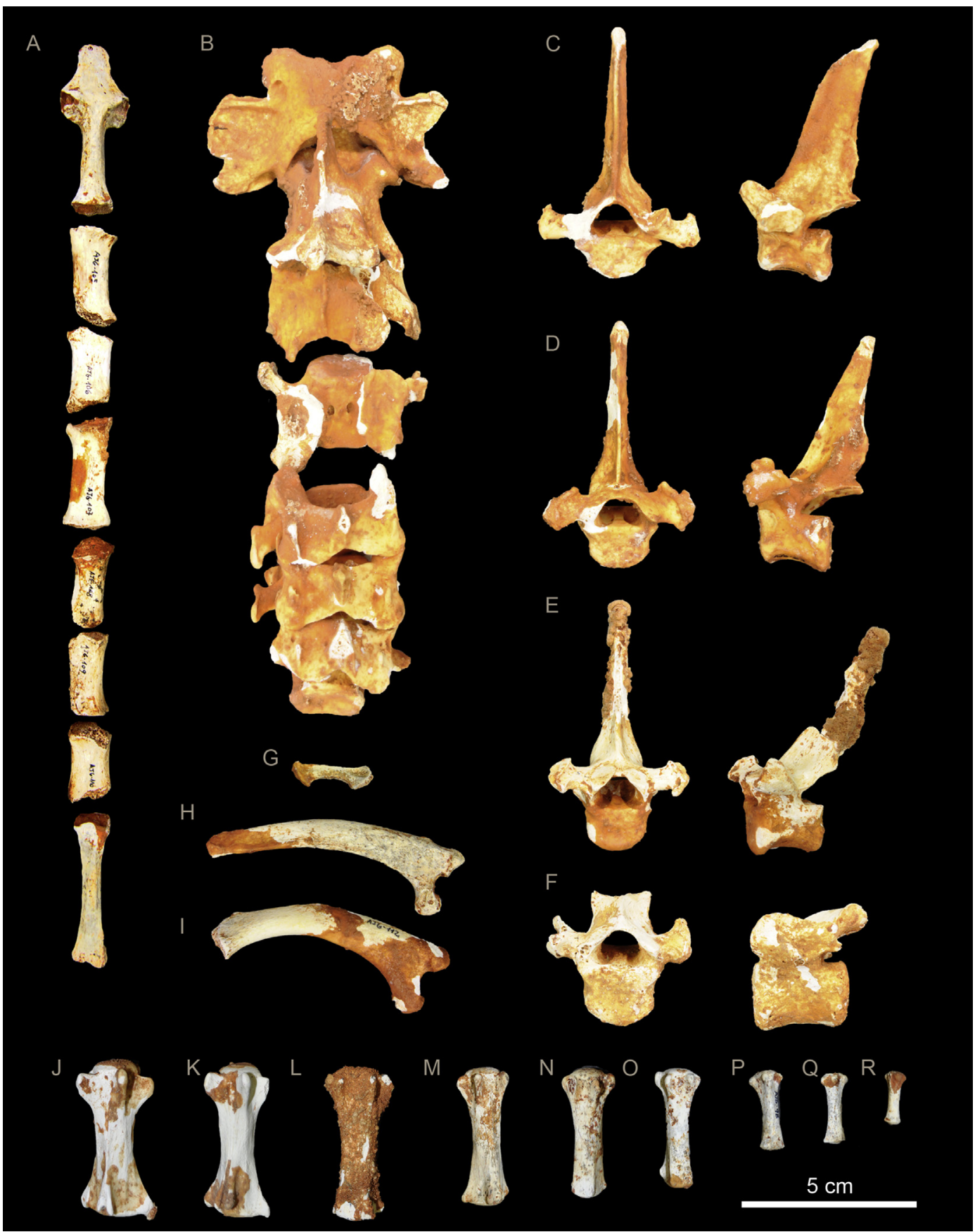

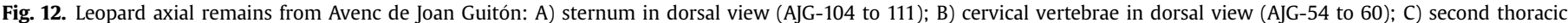

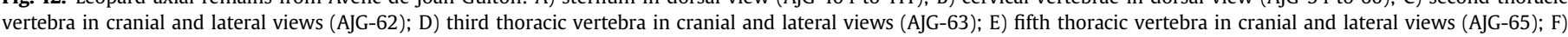

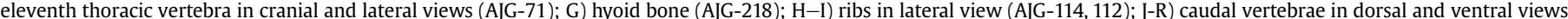
(AJG- 90, 91, 92, 93, 94, 96, 98, 100, 102).

(Fig. 11G). In distal view, the articular facet with the hamate is subrectangular.

The pisiform is subtriangular. The articular surface is divided into two facets; the one that articulates with the pyramidal is subrectangular and that of the hamate is triangular. The posterior process is well developed (Fig. 11H).

The trapezium is rectangular. In proximal view there is a small concave triangular facet that articulates with the scapholunate (Fig. 11I). On the medial surface of the bone there are two small suboval facets, separated by a groove, which articulate with the trapezoid, the capitate and Mc I and II. In distal view, a concave surface articulates with the proximal epiphysis of Mc I.

The trapezoid is subtriangular. In proximal view a lateromedial groove separates the surface into two facets that articulate with the scapholunate (Fig. 11J). On the medial margin, a small trapezoid facet articulates with the trapezium. On the lateral margin, another small rectangular facet articulates with the capitate. In distal view, the convex articulation matches the concavity of the proximal area of Mc II.

The capitate is subrectangular. In proximal view it presents a 


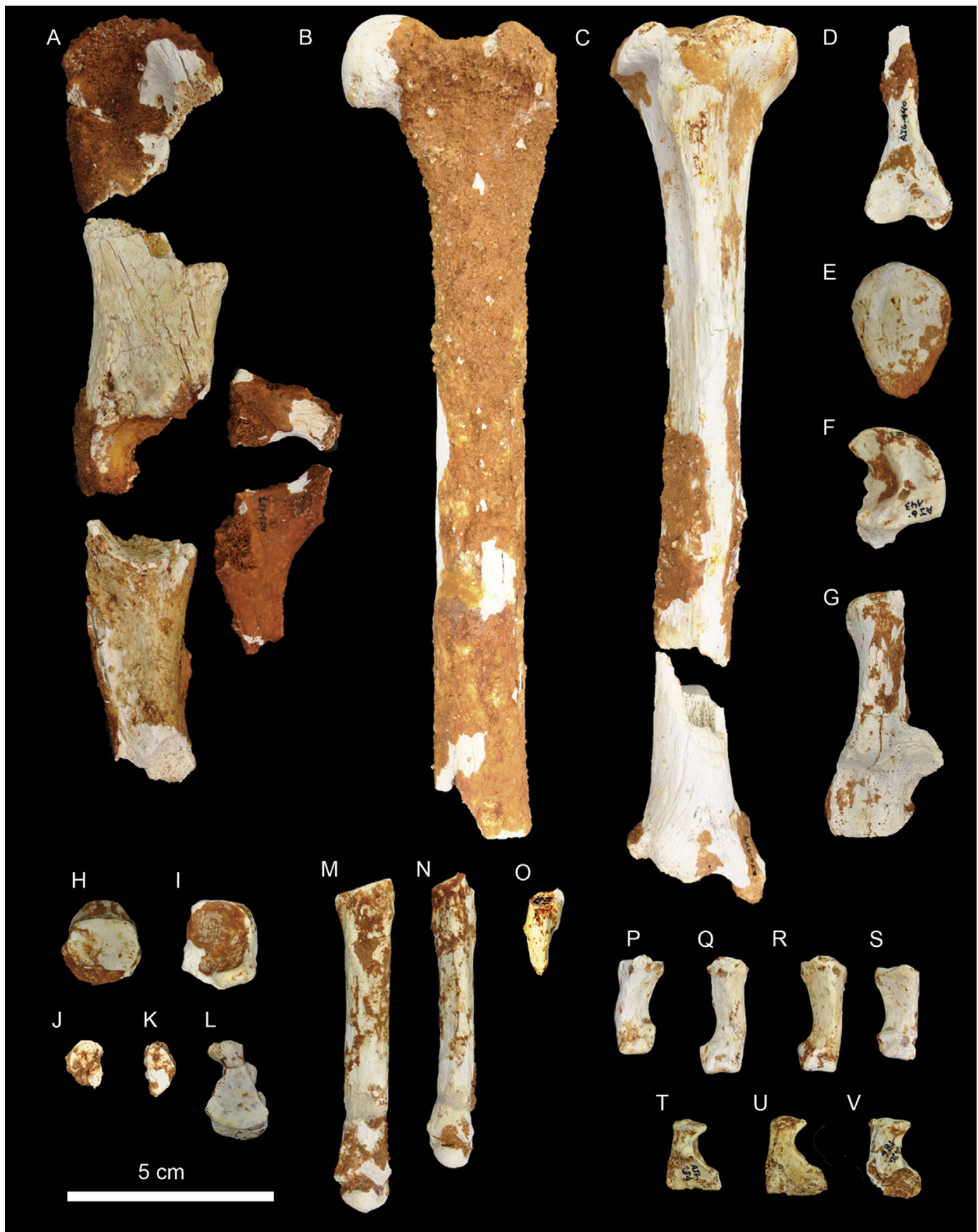

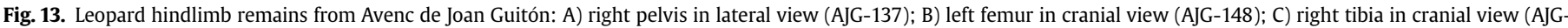

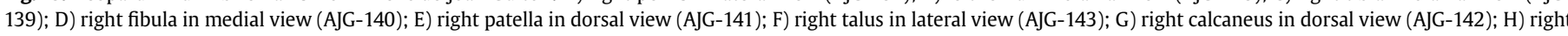

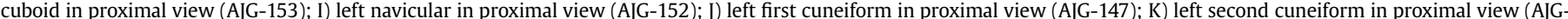

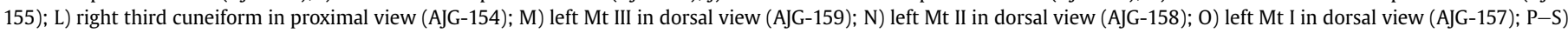
second phalanges in dorsal view (AJG-176, 170, 171, 172); T-V) third phalanges in medial view (AJG-184, 185, 186).

facet that runs obliquely in a dorsopalmar direction, which articulates with the scapholunate and ends in a well-developed posterior process (Fig. $11 \mathrm{~K}$ ). In distal view the articular facet with Mc III is rectangular and concave. On the palmar-lateral margin of this facet is another very small triangular facet that partially articulates with Mc IV. On the lateral side there is a concave surface that serves to articulate the hamate.

The hamate is subtriangular. In proximal view there is a slightly curved, elongated facet that is convex on its dorsal margin and concave on the palmar surface, which articulates with the scapholunate (Fig. 11L). In distal view a suboval concave facet articulates with the proximal epiphysis of Mc IV and partially with that of Mc V. On the medial side there is a subtriangular facet that articulates with the capitate.

Mc I is small and has a subtriangular proximal area. The articulation with Mc II is sinuous. The articular facet with the trapezium is triangular and convex and narrows distally. The diaphysis is very short. The distal articulation projects vertically in an oblique plane 


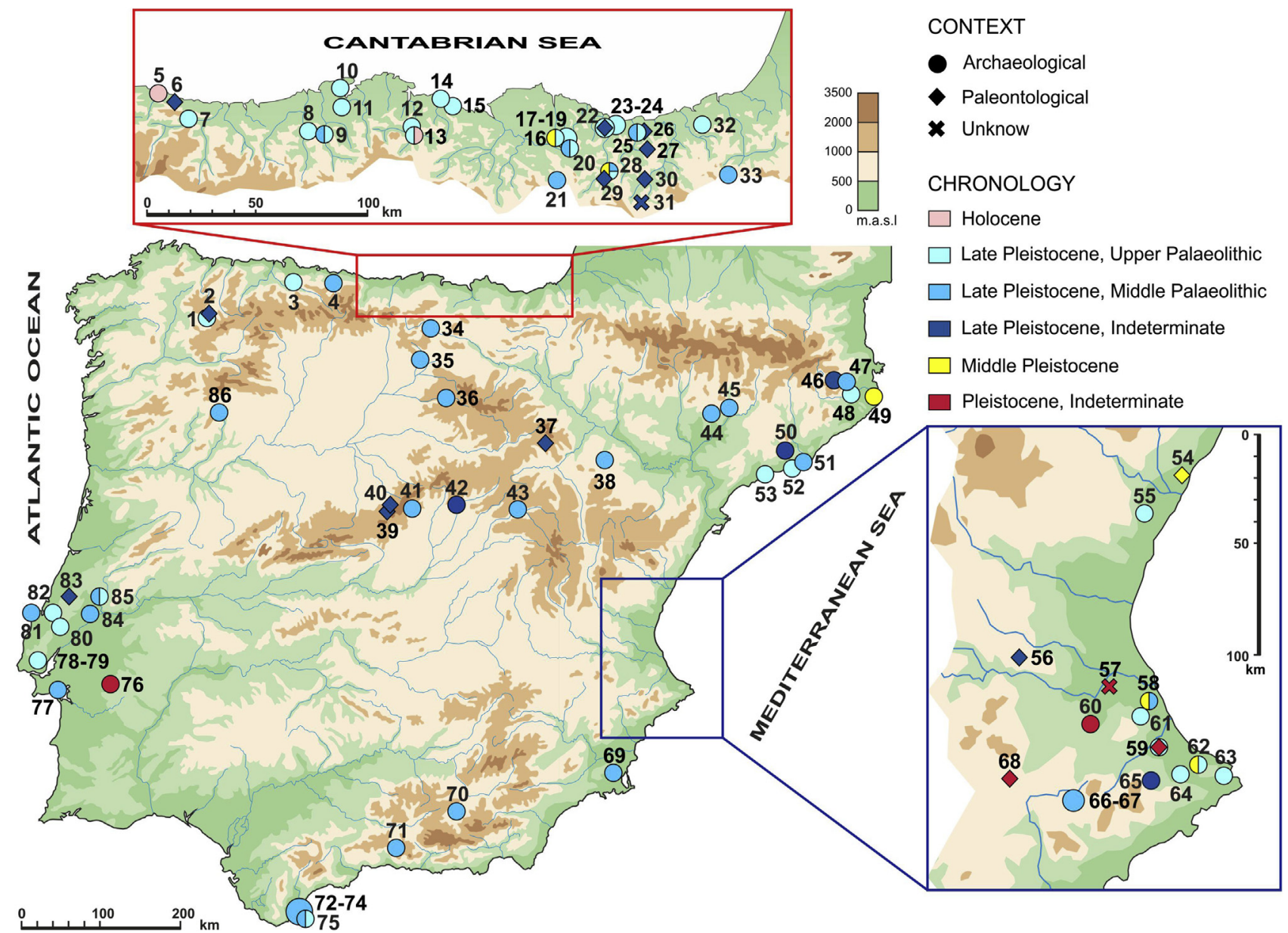

Fig. 14. Palaeogeographic and diachronic distribution of the leopard in the Iberian Peninsula. The numbers refer to the sites of the Table 13.

with a convex articular facet with the first phalanx (Table 7; Fig. 11Q).

In relation to Mc II, the proximal articulation is triangular and slightly angled medially, with a deep dorsopalmar groove. The articular facet with Mc I is oval and slightly concave, like that of Mc III or even deeper, and is typical of P. pardus, whereas in P. uncia it is triangular (Nagel, 1999). The diaphysis presents a triangular cross section in the proximal area that is more circular at the distal end. The distal articulation has a semicircular morphology and is slightly angled medially, with a very prominent sagittal crest on the palmar surface that separates two asymmetrical areas; on the dorsal surface of the distal articulation there are two grooves and two protrusions for insertion of the ligaments. It is a straight bone, except for the distal part, which projects backwards (Table 7; Fig. 11P).

Mc III is very straight and is the longest fore metapodial bone (Table 7; Fig. 110). The proximal articulation is triangular and is divided in two by a dorsopalmar groove. The articular facet with Mc II has a small triangular fossa and an oval one in the articulation with Mc IV. Almost the entire length of the diaphysis has an oval cross section, except for the most proximal part, which is subtriangular. On the dorsal surface of the proximal diaphysis there is a rough surface that fades halfway along the bone. The distal articulation is semicircular, forms a straight line and has a ridge on the palmar surface that separates two symmetrical areas; on the dorsal margin there are two grooves and protrusions for insertion of the ligaments (Nagel, 1999).

The proximal articulation of Mc IV is rectangular-trapezoid and has an oblique surface that is higher on the medial side. The articular facet with Mc III is elongated, while the articular facet with $\mathrm{Mc} \mathrm{V}$ is a deep oval fossa with quite a pronounced ridge on the dorsal margin. The diaphysis is straight and has a circular cross section throughout its length. On the dorsal side of the proximal diaphysis there is a rough surface that disappears towards the middle of the bone. The distal articulation is very similar to that of Mc III (Table 7; Fig. 11N).

Mc V is the smallest of the metacarpal bones (Table 7; Fig. 11M). The longitudinal axis of the bone shows some degree of torsion towards the medial side. The proximal articulation is semicircular. The articular facet with Mc IV has a curved groove with a somewhat pointed protrusion in the centre. The diaphysis has an oval cross section. The distal articulation is very similar to that of Mc II.

The Mc from Valencian sites are very robust if compared with those of the present-day leopard, with similar or greater values in relation to those of $P$. uncia (Table 8), which may be a consequence of adaptation to a mountain environment.

\subsubsection{Axial skeleton}

The sternum is complete and is divided into eight sternebrae (Fig. 12A). The first is elongated and laterally flattened (GL: 54.6) and has a flatter proximal section, where the articular facets with the first pair of ribs are situated (Fig. 12H-I). Sternebrae II to VII are more similar, quadrangular and with wider ends but of varying sizes; IV and V are longer. The last one (VIII) is the longest and thinnest, with two dorsoventrally flattened ends. 
Due to the state of preservation of the vertebrae, only the cervical vertebrae are described (Fig. 12B), and there are few items to compare them with in the Iberian fossil record (Table 9).

The atlas is formed by a ring of bone in the area that joins the dorsal arch to the ventral arch. The cranial articular surface has two cotyloid cavities that articulate with the occipital condyles of the cranium (Indu et al., 2013). It has a circular vertebral foramen. The wings, which are well distinguished from the body and displaced backwards, are rectangular with a rounded edge on the caudal margin. There is a weakly developed tubercle on the dorsal side.

The axis has a dorsoventrally flattened body and is laterally compressed. It has a well-developed odontoid process that almost reaches the occipital bone (Indu et al., 2013). The transverse processes project backwards. The spinous process is ventrally oblique. In ventral view, the bone presents a triangular shape. The caudal articular process is well developed, oblique and projects backwards.

As regards the remaining cervical vertebrae, their body decreases in size from the third to the seventh. The third and fourth cervical vertebrae are poorly preserved. The fifth, sixth and seventh are connected by concretion. In general they present a convex cranial articulation, while the caudal articulation is concave (Indu et al., 2013). The sixth has a poorly developed dorsal spinous process. The morphology of these vertebrae is similar to that of the present-day $P$. pardus.

Only one fragment of hyoid bone remains (Fig. 12G), the basihyoid, a single transverse bony plate in the form of a curved proximodistally flattened arch, with two suboval articular facets at each end. It is the first remains of a hyoid bone preserved in the Iberian fossil record (GL: 24).

\subsubsection{Hindlimb}

The pelvis is highly fragmented (Fig. 13A). The femora are poorly preserved (Fig. 13B). It is a very straight bone, the diaphysis of which has a circular cross section, except at the ends. The proximal articulation has a very spherical head, with an oval fovea capitis. The head is separate from the rest of the articulation and is connected to it by the neck. The greater trochanter is at the same height as the head, while in present-day individuals the head is higher (Podhade et al., 2013). The lesser trochanter is a small tuberosity. Between the greater trochanter and the neck is a deep trochanteric fossa. The dimensions are within the range of Iberian fossils (Table 10).

The proximal end of the tibia has caudal torsion (Fig. 13C). The proximal articulation is triangular and this morphology continues throughout the diaphysis except for the distal portion, where it is more oval. The tibial crest is wide and very prominent. The tibial tuberosity is more receding and is more developed than in the present-day $P$. pardus. The caudal margin of the proximal part has a certain concavity, which is less than that seen in the present-day P. pardus. The caudal surface of the diaphysis has a well-marked popliteal line that runs lateromedially. The distal articulation is subrectangular, with a well-distinguished, vertically projecting medial malleolus. The dimensions are within the range of Iberian fossils (Table 10).

The patella is almond shaped, decreasing in width and thickness from the base to the apex (Fig. 13E). In caudal view it presents two articular surfaces; the lateral surface is wider (Table 10).

A proximal fragment of talus remains (Fig. 13F). The margins of the trochlea are subparallel; in distal view the lateral margin projects and is more developed. The concavity of the trochlea is dissymmetrical in distal view. The tarsal sinus is deep and separates the articular surface with the calcaneus into two areas (Podhade et al., 2014b). The width of the trochlea is average for Iberian leopard fossils.
The plantar border of the body of the calcaneus is curved and wide, while the dorsal border is narrower and more pronounced. The tuber calcanei is quite well developed and becomes thicker at the proximal end, with a groove for the Achilles tendon. This area is asymmetrical as in $P$. pardus, whereas in $P$. uncia it is symmetrical. The coracoid process is subrectangular and somewhat convex. The articular facet with the talus is oval and concave, like the distal articulation with the cuboid. The dimensions are small, similar to other possibly female Iberian fossils (Cova Negra and Furninha) (Table 10; Fig. 13G).

There are very few other tarsal bones in the Iberian fossil record (Table 10).

The cuboid has a trapezoid shape. In proximal view there is a flat rectangular facet that articulates with the calcaneus (Fig. 13H). On the medial margin there is a small triangular facet that articulates with the navicular. In distal view, a concave oval surface articulates with the proximal epiphysis of Mt IV and V.

The navicular is suboval and quite flat except for the posterior process, which projects proximally. In proximal view we see the articulation with the talus, which is very concave (Fig. 13I). In distal view, the surface is flatter and there are two facets separated by a triangular fossa for the articulation of the cuneiform bones; on the lateral margin there is a larger semicircular facet for the third cuneiform, and on the lateral surface there is a smaller, subrectangular facet for the second cuneiform.

The first cuneiform in proximal view presents a semicircular concave facet that articulates with a small posterior facet of the navicular (Fig. 13J). In distal view it presents an elongated facet that articulates with Mt I.

The second cuneiform is subrectangular and is the smallest tarsal bone. In proximal view it has a somewhat concave oval facet that articulates with the navicular (Fig. 13K). In distal view, a flatter, elongated, semicircular facet articulates with the proximal part of Mt II.

The third cuneiform is subtriangular. In proximal view there is a concave triangular surface that articulates with the navicular and that ends in a distally projecting posterior process (Fig. 13L). In distal view another flatter triangular facet serves as an articulation for Mt III.

It has been possible to describe Mt I, II and III.

Both Mt I have been preserved, which constitutes the first citation of this in the Iberian fossil record. It is a small triangular residual bone (Fig. 130). It decreases in width from the proximal to the distal end. In proximal view there is a flat oval facet that articulates with the first cuneiform. It flattens in the distal direction and ends with a rounded edge.

The main axis of Mt II shows medial torsion (Fig. 13N). The proximal articulation is triangular and runs obliquely in dorsal view. The articulation with Mt I (oval concavity) is on the medial surface of the proximal area. On the lateral side, the articulation with Mt III forms two suboval fossae. The distal articulation is semicircular, forms a straight line and has a ridge on the plantar margin that separates two asymmetrical areas; on the dorsal margin there are two grooves and two protrusions for insertion of the ligaments.

Mt III has a dorsoplantarly flattened and transversely widening diaphysis. The articulation with Mt II is an oval fossa flanked by two protrusions. The articulation with Mt IV is a deeper triangular fossa. The proximal end has a characteristic "T" shape, while the distal articulation is semicircular, forms a straight line and has a ridge on the plantar surface that separates two symmetrical areas; on the dorsal surface there are two grooves and two protrusions for insertion of the ligaments (Fig. 13M).

The metatarsals exhibit significant robustness (Tables 8 and 11), greater than the values for $P$. pardus and similar to or greater than 
P. uncia, as suggested by Nagel (1999) and Sauqué et al. (2014a), which has been associated with a process of convergence between Pleistocene leopards and $P$. uncia because they inhabited a similar environment.

\subsubsection{Phalanges}

All the first phalanges have been described except for the posterior phalanges of digit $\mathrm{V}$ (Table 12). In general, the first phalanges of the hindlimb are longer and more robust, with the exception of the forelimb phalanges of digits III and IV, which are somewhat longer. In lateral view they all present the characteristic curvature.

The longest of the first phalanges of the forelimb (Fig. 11R-V) are those of digit III, followed by those of digits IV, V, II and I. The vestigial first phalanx of digit I is a short bone with an oval, medially oblique proximal articulation. The distal articulation is wide. The first phalanx of digit II is narrow with some curvature, the proximal articulation has a half-moon shape and the distal articulation tapers, both are asymmetrical and have a more developed lateral side; their body has a circular cross section. The first phalanx of digit III is large, with a dorsopalmarly flattened body and a wide, half moon-shaped proximal joint; the distal articulation is straight and symmetrical, with a deep distal trochlea. The first phalanx of digit IV is very similar to that of digit III but is shorter and thinner. The first phalanx of digit $\mathrm{V}$ is similar to that of digit II but with torsion in the opposite direction, and the ends are more developed on the medial side than on the lateral side.

The morphology of the first phalanges of the hindlimb is similar to that of the forelimb but they are more robust.

The second phalanges of the forelimb (Fig. 11W-Z) are longer than those of the hindlimb (Fig. 13P-S). The articulation is also wider in those of the forelimb, but the width of the body is greater in those of the hindlimb. As in the first phalanges, the longest phalanges are those of digits III and IV. The second phalanges have a very curved body with a triangular cross section that ends in a trochlea that is laterally displaced to allow lateral retraction of the third phalanx. The half moon-shaped proximal articulation is a concavity separated by a ridge that serves as an articulation with the first phalanx. There is quite a deep fossa on the plantar/palmar border of the proximal articulation. In the second phalanges of the forelimb the most well-developed articular process is that of digit II, while it is digit III in the hindlimb.

Six right third phalanges and all the left ones (five of the forelimb and four of the hindlimb) have been preserved. In general the forelimb ones (Fig. 11AA-AE) are larger and more robust than the hindlimb ones (Fig. 13T-V); in the forelimb those of digits II, III and IV are largest, while those of digits III and IV are largest in the hindlimb. The articular surface with the second phalanx is deep and oval.

\subsection{Cova de les Malladetes}

The right pelvis is almost complete and very well preserved (Table 10; Fig. 4A). The acetabulum, which is very circular, is fused, although the iliac crest and ischial tuberosity are not yet fused (late fusion). The bone is very straight. The ilium is subrectangular and elongated, and the wing has a curved border. In medial view, the auricular surface is divided into two areas by a triangular fossa, the dorsal one larger than the ventral. The ischium is shorter than the ilium and is slightly twisted, while the ilium is on a straight plane. The ischium has a very thin cranial border, while the caudal border is wider. The characteristics coincide with those of the pelvis of present-day leopards in India (Podhade et al., 2014a), although the one found at Malladetes is longer.

\subsection{Cova del Racó del Duc}

The leopard remains found at this site show morphological similarities to AJG. We therefore describe some elements that are poorly preserved or not found at AJG (talus and Mt IV and V). In REC I the remains correspond to two individuals, one senile and larger than the other, and in REC II to a third, large individual.

In REC I, the set of large remains comprise tibia, canine, carpal, patella, four metacarpals and two metatarsals. The smaller remains that may correspond to a female (radius, talus, metacarpal and metatarsal) have similar dimensions to AJG (Fig. 5). It has not been possible to attribute the other elements to a specific individual (caudal vertebrae, phalanges and ribs). All the remains from REC II are large (Fig. 6).

Two talus bones are described, one large one from REC II (Fig. 60) and another smaller one from REC I (Fig. 5X). The margins of the trochlea are subparallel, but the lateral margin projects and is more developed. The concavity of the trochlea is dissymmetrical. Both features appear in AJG. The neck is not very elongated and the head is medially oblique. The trochlea and neck are not aligned. The head is oval and has a bony ridge. In plantar view, the sinus is deep and separates the articular surface with the calcaneus into two areas. The dimensions of REC I are very similar to those of AJG and Bolinkoba. The talus from REC II is among the largest in the Iberian fossil record, similar to the more robust examples from Zafarraya (Table 10).

Two Mt IV are described from two large individuals, one larger and more robust (Fig. 6Y) than the other (Fig. 5V). The bone is large and straight, with some torsion towards the lateral side. The proximal epiphysis is rectangular. The articulation with Mt III has two oval facets separated by a deep groove, while that of Mt V is a very deep oval fossa. The cross section of the diaphysis is more quadrangular at the proximal end and becomes more oval towards the distal end. The distal articulation is semicircular, forms a straight line and has a ridge on the plantar border that separates two symmetrical areas; on the dorsal surface there are two grooves and protrusions for insertion of the ligaments. The measurements of the larger individual are similar to those of the robust forms of Zafarraya (Table 11).

Two Mt V are described, one more slender (Fig. 5W) and the other more robust (Fig. 6Z). The bone is curved in a dorsoplantar direction and is the smallest of the functional metatarsals. The diaphysis has a round cross section. The proximal epiphysis is triangular. The articulation with Mt IV consists of a pointed oval projection. In plantar view the proximal area presents two lobes (forked). The distal joint, which is displaced laterally, is semicircular and has a ridge on the plantar border that separates two asymmetrical areas. The dimensions are consistent with those of other specimens from sites in the Iberian Peninsula (Table 11).

\subsection{Cova del Bolomor}

The morphology, dimensions and robustness of the Mt III (Fig. 7C) found in level XIII (Middle Pleistocene) coincide with those of other Middle and Late Pleistocene remains from the Iberian Peninsula (Tables 8 and 11).

\subsection{Cova Negra}

A calcaneus found in the 2013 excavation presents the same morphometric characteristics as AJG and may correspond to a female (Table 10; Fig. 7F). A P4 from an adult and a p4 from a 1.5- to 2year old individual are presented (Stander, 1997) (Tables 4 and 5), but not described (Pérez, 1977).

The P4 has a very high crown, a well-differentiated rounded 
parastyle and a paracone or main crest with a well-marked, vertical anterior ridge (Fig. 7I). The posterior edge of the paracone and that of the metacone form a narrow angle. The cusp of the paracone presents some wear. The protocone is rounded and well developed, a typical characteristic of Late Pleistocene European leopards. The mesial-distal outer length is similar to that of Escoural and Aintzulo, but greater than for the females of AJG and Algar da Manga Larga (and possibly Torrejones) and that of present-day leopards.

p4 has a poorly differentiated, reduced paraconid, unlike that of AJG, similar to Caune de l'Arago (Testu, 2006). There is a welldeveloped almost vertical protoconid that shows no wear. The posterior cusp is reduced like the anterior one. Behind the posterior cusp there is a well-marked cingulum on the lingual side. In occlusal view, the tooth is widest in the distal third, with some narrowing in the middle (Fig. 7J). The tooth has a mixture of archaic features (Middle Pleistocene) and others that are more typical of present-day and Late Pleistocene individuals. The dimensions coincide with those of some Late Pleistocene (Los Rincones and Algar da Manga Larga) and present-day leopards.

\section{Discussion}

\subsection{Pleistocene Panthera pardus in the Iberian Peninsula}

The new cranium found at AJG, together with those from Allekoaitze and Aintzulo in the north of the Iberian Peninsula (Mariezkurrena, 2011; Corral, 2012; Altuna and Mariezkurrena, 2013), Algar da Manga Larga in Portugal (Cardoso and Regala, 2006), Equi in Italy (Ghezzo and Rook, 2015) and Vjetrenica in Bosnia-Herzegovina (Diedrich, 2013) represent Late Pleistocene European leopards. Although the remains from AJG could not be dated, the cranium is very similar to that of other European specimens from the Late Pleistocene, so it is provisionally attributed to that period. The cranium of these leopards is of a medium length, with wide nasal and frontal areas, a globular neurocranium and variable dimensions that for Diedrich are associated with strong sexual dimorphism, which would explain the existence of more elongated morphotypes in males (close to those of African leopards) and shorter morphotypes in females (more similar to P. uncia), corresponding to the subspecies P. pardus spelaea (Diedrich, 2013). For Ghezzo and Rook (2015), the crania of male and female P. pardus differ in terms of their morphological features and size is a criterion that can be more variable.

In this regard, and in the light of new work, the material from Los Rincones could be reassigned to $P$. pardus, although the remains present similarities with the subspecies spelaea described by Diedrich (2013).

The mandibles of Late Pleistocene $P$. pardus specimens from the Iberian Peninsula are longer than those of $P$. uncia, with a mixture of features of both species, as seen at Los Rincones (Sauqué and Cuenca-Bescós, 2013).

The dentition of Late Pleistocene $P$. pardus from the Iberian Peninsula is similar in size to that of present-day leopards. P4 has a very hypsodont crown. For Diedrich (2013), the morphology of the upper carnassial has undergone a process of evolution across the different European leopards. The height of the crown increases from the Middle Pleistocene subspecies to P. pardus spelaea, $P$. pardus ciscaucasica and, in particular, $P$. uncia. The presence of a rounded protocone is characteristic of the last European Pleistocene leopards and P. uncia, an area that tends to decrease from ancient forms to the end of the Late Pleistocene. In general, the morphology of P4 in Late Pleistocene leopards has similarities with P. uncia (Sauqué et al., 2014a).

The Late Pleistocene $P$. pardus of the Iberian Peninsula is a leopard that has similarities with $P$. uncia. The specimens found at
Algar da Manga Larga, Los Rincones, AJG, Allekoaitze, Aintzulo and Zafarraya (Sauqué et al., 2014a) present uncioid features and some of them have been reassigned by Diedrich (2013) to the subspecies spelaea. This could be related to the adaptation of both species to a mountain habitat, showing that these species may have a similar ethology.

Variations in size between the different leopard remains found in the Iberian Peninsula may be a consequence of both sexual dimorphism and climatic factors, as it is very likely that they were larger during cold phases than in milder periods (Diedrich, 2013). In any case it should be taken into account that there may be some overlap between the dimensions of males and females; some authors therefore believe it is better to assign the sex on the basis of morphological rather than metric criteria (Ghezzo and Rook, 2015).

As regards the age structure of the leopards, almost all the material found to date in the Iberian Peninsula corresponds to adult individuals, which is why we believe it is more plausible that they used the caves as storage places to protect their catches from other predators (de Ruiter and Berger, 2000; Diedrich, 2013; Sauqué et al., 2014c). This scenario is consistent with leopard breeding patterns, as they do not use caves (Diedrich, 2013). The presence of cubs in the Iberian Peninsula record is limited to one deciduous tooth at Jou Puerta (Álvarez-Laó, 2014), to which the m1 germinal from Malladetes has now been added. These remains may indicate a possible use as breeding areas, as is the case of Equi (Ghezzo and Rook, 2015) and Vraona (Nagel, 1999).

During the Late Pleistocene, leopards in the Iberian Peninsula fed mainly on small ungulates, particularly Capra pyrenaica, a species that inhabits rocky areas. The association between leopards and Spanish Ibex is present in many Pleistocene sites such as Los Rincones (Sauqué et al., 2014c), Zafarraya (Barroso et al., 2006), Amalda (Yravedra, 2010a) and Racó del Duc (currently being studied). For their part, snow leopards are also specialised in domestic or wild goats (Anwar et al., 2011; Diedrich, 2013), prey that they do sometimes carry to their dens (Oli et al., 1993; Diedrich, 2013).

As regards leopard remains found in Middle Pleistocene sites of the Iberian Peninsula (Cueva de Lezexiki, Cueva de Arlanpe, Cau del Duc d'Ullà, Cau d'En Borràs, Cova del Corb and Cova del Bolomor), as in the rest of Europe there are few specimens and no good morphometric descriptions. For Diedrich (2013) the remains from this period correspond to the subspecies P. pardus antiqua Cuvier 1835, which migrated to Europe about $300 \mathrm{ky}$ BP. In order to confirm the existence of different Pleistocene subspecies in Europe (Diedrich, 2013) or that of a single chronospecies with significant variability (Ghezzo and Rook, 2015), we would need a larger record of leopard remains in Middle and Late Pleistocene contexts.

In our case, despite the characteristics of Mediterranean leopards being very similar to those presented by Diedrich (2013) and Sauqué et al. (2014a) for $P$. pardus spelaea, in addition to having certain similarities or common features with $P$. uncia, we believe that, in view of the recent publication by Ghezzo and Rook (2015), intraspecific variability may also account for the variations seen in the leopards of the Iberian fossil record and we therefore classify the remains as $P$. pardus.

\subsection{Diachronic and palaeogeographic distribution of the leopard in the Iberian Peninsula}

The earliest presence of the leopard in the Iberian Peninsula corresponds to the Middle Pleistocene levels of Lezetxiki (234 ky). The few citations from this phase are distributed throughout the Mediterranean and Cantabrian regions (Table 13; Fig. 14). In some cases (Cova del Mollet I), the initial determinations (Maroto et al., 1987) have not been confirmed (Maroto et al., 2012). There are 
even two possible remains from the transition between the Lower and Middle Pleistocene that would push back the leopard's appearance in the Iberian Peninsula until this time, a calcaneus (Panthera cf. pardus) from Venta Micena (Pons-Moyà, 1987) and a metapodial (Panthera sp.) from Gran Dolina (García, 2003), but they do not have a clear assignation. The leopard's presence in the Iberian Peninsula appears to be later than in the rest of Europe (Sauqué and Cuenca-Bescós, 2013).

The vast majority of references from the Iberian Peninsula come from the Late Pleistocene (Table 13; Fig. 14), mainly during MIS 2 (49.4\%) and MIS 4-3 (45.9\%), while there are few references from the Early Late Pleistocene or MIS 5e (4.7\%). The information collected enables us to distinguish three points during MIS 2. Most references come from the beginning of this period and coincide with the Early Upper Palaeolithic (25.9\%), with fewer citations during the Solutrean/LGM (11.8\%) and Magdalenian (4.7\%). This tells us that the phase of greatest distribution of the leopard in the Iberian Peninsula coincides with Mousterian Neanderthal occupations and early modern humans from the beginning of the Upper Palaeolithic. The leopard's ubiquistic nature explains its presence in both harsh phases and times when the climate was milder.

Two very late records in the Cantabrian region (Cueva de las Pajucas and Cueva de la Riera) suggest that the species survived in Europe into the Early Holocene (Altuna, 1972; Álvarez-Laó, 2003). These Holocene references together with those from the end of the Lateglacial period confirm the idea that the species survived for longer in the Iberian Peninsula than in other parts of Europe (LGM), mainly in the Cantabrian region (Sauqué and Cuenca-Bescós, 2013).

The main areas of distribution of the species in the Iberian Peninsula are areas of karst relief near the coast, with many of the findings concentrated in the eastern part of the Cantabrian coast, the Catalan coastal mountain range, the foothills of the Betic system close to Cabo de la Nao in the Mediterranean and Portuguese Estremadura. The rest of the peninsula, which has fewer or no records, has little karst development and preservation of the remains is difficult. The new findings from the Mediterranean region confirm a particularly dense distribution of the leopard, very similar to that of the Cantabrian region, during the Late Pleistocene.

The leopard's distribution can be analysed in relation to the two broad biogeographic regions of the peninsula, the Euro-Siberian and Mediterranean regions (Rivas-Martínez, 1987; Rivas-Martínez et al., 2002). The sites with records in the Euro-Siberian region are mainly located below 600 masl (approx. 79\%) and secondarily between 540 and 780 masl. The sites at a higher altitude in the region (Arrillor and Eirós) are barely more than 700 masl. As regards the Mediterranean region, citations in the east of the peninsula are concentrated in areas below 200 masl and in sites near the coast, while on the Portuguese Atlantic coast they reach 460 masl (approx. 44\%). Thirty-seven per cent of the findings in this Mediterranean region appear in areas between 115 and 1000 masl. Nineteen per cent of the records correspond to places at a higher altitude in the mountains of the Iberian and Guadarrama mountain ranges, except for Lorga de Dine, in the Portuguese part of the Montes de León. These are approximate estimates and are based on the current sea level, and it must be kept in mind that Pleistocene transgression-regression episodes would have affected the coastline (Álvarez-Laó and García, 2010, 2011).

The leopard is a common taxon in the Iberian Peninsula during the Late Pleistocene, although in general there are a small number of remains compared with other carnivores (Sauqué and CuencaBescós, 2013; Sauqué et al., 2014a). This is due to their solitary, territorial behaviour, with large areas of distribution (Nowell and Jackson, 1996; Kingdon, 1997; Turner and Antón, 1997). Another factor that influenced the frequency of this taxon in cave sites was the high degree of competition with other carnivores during the
Late Pleistocene. $P$. pardus had to compete with the hyena (Crocuta crocuta spelaea), the cave lion (Panthera leo spelaea), the wolf (Canis lupus), the dhole (Cuon alpinus) and with two species of bear, the cave bear (Ursus spelaeus) in the northern half of the Iberian Peninsula and the brown bear (Ursus arctos) in the rest of the territory (Diedrich, 2009, 2011; Villaluenga, 2009; Pérez et al., 2010; Morales et al., 2012; Sauqué et al., 2014a).

During the Late Pleistocene the leopard was distributed throughout the Iberian Peninsula, and it is the part of Europe with the highest concentration of remains. This may be due to the fact that there were fewer carnivores such as hyenas and lions than in other areas of Europe (Diedrich and Žák, 2006; Diedrich, 2007, 2011, 2014). In the Cantabrian region most of the caves were occupied by bears during the Late Pleistocene (Villaluenga, 2009). Bears and hyenas were less common in the Mediterranean region, meaning that leopards competed mainly with humans and dholes (Pérez et al., 2010; Morales et al., 2012) for the use of caves, which resulted in the Mediterranean area having a significant density of sites containing leopard remains (Fig. 14).

\subsection{Contexts of appearance and origin of the leopard remains}

During the Pleistocene in the Iberian Peninsula there was interaction between hominins and felines: from the lion (Blasco López et al., 2010), leopard (Arribas, 1997; Camarós et al., 2015) and different species of lynx (Yravedra, 2005) to the wild cat (Gabucio et al., 2014). Hominins hunted them for their meat, skin and bones, as well as to defeat a direct competitor for prey and for the use of caves and shelters (Brugal and Fosse, 2004). Hominins would also have been prey to some of these carnivores (Kruuk, 2002; Camarós et al., 2015).

In the case of leopards, these processes can be seen in several sites of the Iberian Peninsula. Sometimes the bones found in archaeological contexts, mostly as isolated elements, exhibit cut marks, indicating that these animals were hunted and/or processed by humans. For example, the incisions on the cranium of the leopard discovered at Torrejones (Guadalajara), which was found in the Middle Palaeolithic level, were made while the skin was being removed (Arribas, 1997). The same interpretation has been made of the marks observed on a leopard metapodial from Cova Foradada (Alacant) belonging to an Early Upper Palaeolithic level (Pantoja et al., 2011). On examination of a partially burnt Mt V from Cova Negra (CN 10952; Pérez, 1977), several cut marks were identified on the dorsal surface of the diaphysis, which could also be from skinning (Fig. $7 \mathrm{H}$ ). Other evidence includes a leopard ulna from Cova Foradada, from the same level as the aforementioned metapodial, which shows intentional polishing on the distal end that could correspond to a bone awl (Pantoja et al., 2011). The remains found at Sima de las Palomas (Murcia) included a burnt leopard bone and various articulated appendicular elements associated with human remains, and it is possible that the Neanderthals hunted them (Walker et al., 2012). The leopard remains found at Llonín (assemblage II from the "Cono Posterior" section), which are currently being studied, do not present direct signs of human action, but they could be placed within a context that is considered anthropogenic. It is a partial leopard skeleton surrounded by five carefully arranged stalactite fragments and associated with other faunal and lithic remains (Fortea et al., 1999). In this case the leopard remains may be related to symbolic behaviours or acts.

Leopard attacks on humans can be seen as another type of interaction. Recently, a possible leopard attack on a Neanderthal has been documented in Cova Negra. Two punctures on a parietal fragment bone are likely to have been produced by two canines of a carnivore (Camarós et al., 2015).

Apart from predation, other interaction processes can also occur 


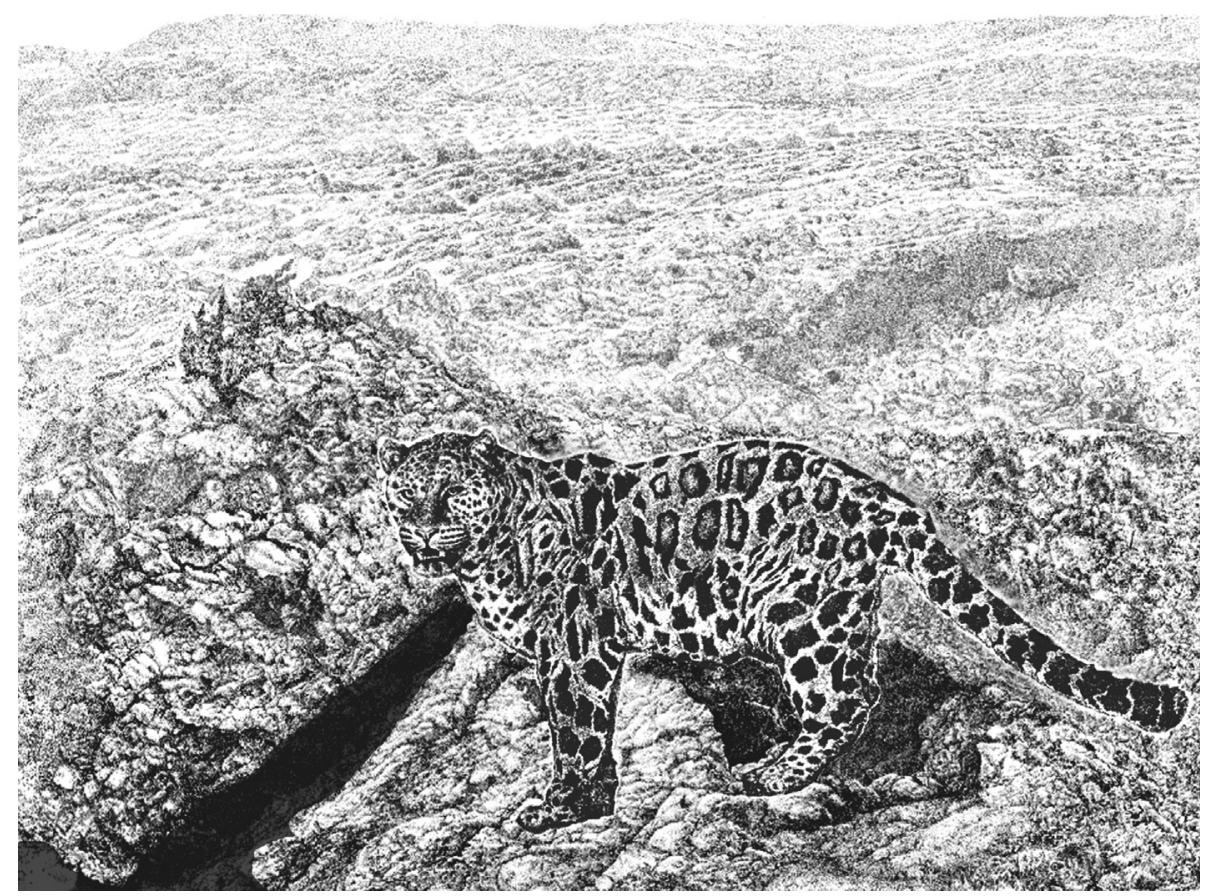

Fig. 15. Leopard (Panthera pardus) close to the Avenc de Joan Guitón chasm (Illustration of Gianfranco Messi).

when leopards occupy the same locations as humans (breeding dens, shelters, feeding or storage areas) and carry their prey there, creating bone accumulations that could be mixed with materials from anthropogenic sources (for example, in the Iberian Peninsula, Sauqué et al., 2014b). The latter may have also been scavenged by leopards as well. The phenomenon of prey being transported to caves by leopards has been documented in several areas (de Ruiter and Berger, 2000). Sometimes, leopard remains of natural origin are associated or interspersed with archaeological material during phases when humans did not occupy the sites, as is the case of Abric Romaní (Cáceres et al., 1993) or Furninha (Brugal et al., 2012). In palaeontological contexts, the accumulations are natural and result from predation or natural death as in AJG (Fig. 15), in this case a complete skeleton, where the fragmentation is perimortem and post-depositional.

In the Valencian region (central Mediterranean area of the Iberian Peninsula), there are few leopard remains in the archaeological contexts, with the exception of some Early Upper Palaeolithic levels of sites like Cova Foradada (Pantoja et al., 2011) and Malladetes (this study). These were times when there was little human presence in the area, with small or highly scattered groups and a great proliferation of carnivores, such as those documented in other areas of the Iberian Peninsula (Valente, 2004a,b). For some authors, this scenario may be due to a hiatus when humans were not present that coincided with the disappearance of the last Neanderthals and the arrival of the first anatomically modern humans (AMH) (Wood et al., 2013; Galván et al., 2014). However, it could also be a reflection of the low population density at the beginning of the Aurignacian expansion in the Iberian Mediterranean, as archaeological contexts bear witness to the interaction of humans and felines in the same caves.

\section{Conclusions}

The Mediterranean leopards presented in this paper have morphological characteristics similar to those of other European remains that have been attributed to the subspecies $P$. pardus spelaea, with features representing a mixture between the presentday leopard and the snow leopard, showing strong sexual dimorphism. Despite this morphological similarity, with so few remains and no genetic analysis of Pleistocene leopard populations to corroborate or refute the existence of subspecies, we consider it prudent to classify the remains at species level (P. pardus).

The leopard skeleton found at AJG is the best preserved Pleistocene specimen of this kind in the Iberian Peninsula and one of the most complete specimens in the European and global fossil record. We believe it to be a reference for future studies. In this regard, we present an exhaustive morphological description of the cranial and postcranial elements that will be of great use, as leopard remains are usually found fragmented and it is difficult to establish the relationship between the different features of the cranial and postcranial skeleton.

The greatest expansion of the leopard in the Iberian Peninsula took place during the Late Pleistocene, between MIS 4 and MIS 2. Moreover, the species survived for longer in this geographical region than in other areas of Europe, with records from the Lateglacial period, possibly even reaching the Early Holocene.

Our review of the leopard's distribution in the Iberian Peninsula includes many new records, of which there are now 86, making the Iberian Peninsula one of the areas with the highest density of sites containing Pleistocene leopard remains in Europe.

The most complete and best preserved leopard remains in the Iberian Peninsula appear in palaeontological sites, but such findings are in the minority. The majority of leopard records correspond to Middle and Upper Palaeolithic archaeological contexts. In this case there are signs of processes of interaction between these carnivores and prehistoric human groups, Neanderthals and early AMH, based on competition, feeding and other behaviours.

\section{Acknowledgements}

With respect to the finding, recovery and restoration of the skeleton from AJG and the analyses, photographs, video, X-rays and scanning of the remains, we would like to thank: Àngel Cambra, 
Josep A. Ribera, Juan Salazar, João Zilhão, Mara Peiró, Juan Navarro, Trinidad Pasíes, María Perales, Clínica Dental Adeslas, José Madrid, Elvira Aura, Mari Carmen Díaz and Xerea Films.

Various investigators have provided us with information about leopard remains in the Basque Country (Asier Gómez-Olivencia), Asturias (Javier Fortea and Marco de la Rasilla), Catalonia (Jordi Nadal, Juan Ignacio Morales, Antonio Rodríguez-Hidalgo and Palmira Saladié), Valencia (Inocencio Sarrión, Josep Fernández, J. Emili Aura, Joaquim Juan-Cabanilles, Bertila Galván, Cristo Hernández, Juan V. Morales, Leopoldo Pérez and Rafael Martínez), France (Agnès Testu and Jean Philip Brugal) and Portugal (João L. Cardoso). A particular thanks to palaeoartist Gianfranco Mensi for his precise reconstruction. Thanks to Jaime Vives for the revision of the text.

The text has been translated into English by Grace Horsley and Jordi Sanchis.

We would like to thank Helena Bonet, the director of the Museu de Prehistòria de València, for the support and interest she has shown for this project.

This work forms part of the following projects: (HAR-201124878 ) "Paleolítico medio final y Paleolítico superior inicial en la región central mediterránea ibérica (Valencia y Murcia)" (End of the Middle Palaeolithic and beginning of the Upper Palaeolithic in the central Mediterranean region of the Iberian Peninsula (Valencia and Murcia)) and (PROMETEOII/2013-016) "Más allá de la Historia. Origen y consolidación del poblamiento paleolítico valenciano" (Beyond history. Origin and consolidation of the Valencian Palaeolithic settlement).

The authors also thank the reviewers for the useful suggestions that greatly improved the manuscript. Finally, the authors thank the work of the editorial team of Quaternary Science Reviews.

\section{Appendix A. Supplementary data}

Supplementary data related to this article can be found at http:// dx.doi.org/10.1016/j.quascirev.2015.07.013.

\section{References}

Alférez, F., Molero, G., Maldonado, E., Bustos, V., Brea, P., Buitrago, A.M., 1982 Descubrimiento del primer yacimiento cuaternario (Riss-Würm) de vertebrados con restos humanos en la provincia de Madrid (Pinilla del Valle). Colpa 37, 15-32.

Alonso, S., Vaquero, M., Ameijenda, A., 2014. La Cova de Valdavara y Valdavara 3 (Becerreá, Lugo). In: Sala, R., Carbonell, E., Bermúdez de Castro, J.M., Arsuaga, J.L. Eds.), Los cazadores recolectores del Pleistoceno y del Holoceno en Iberia y e Estrecho de Gibraltar. Estado actual del conocimiento del registro arqueológico. Universidad de Burgos y Fundación Atapuerca, pp. 31-33.

Altuna, J., 1971. Los mamíferos del yacimiento prehistórico de Morín (Santander). In Cueva Morín. Excavaciones 1966-1968. Publicaciones del Patronato de las cuevas prehistóricas de la provincia de Santander, vol. VI, pp. 367-399.

Altuna, J., 1972. Fauna de mamíferos de los yacimientos prehistóricos de Guipúzcoa. Munibe, p. 24

Altuna, J., 1973. Fauna de mamíferos del yacimiento prehistórico de Los Casares (Guadalajara). In: Barandiarán, J.M. (Ed.), La Cueva de los Casares, pp. 97-116.

Altuna, J., 1980. Fauna de Axlor. Campaña de 1971. In: Barandiarán, J.M. (Ed.), Obras Completas, vol. 17, pp. 273-279. Bilbao.

Altuna, J., 1990. Caza y alimentación procedente de macromamíferos durante el Paleolítico de Amalda. In: Altuna, J., Baldeón, A., Mariezkurrena, K. (Eds.), La Cueva de Amalda (Zestoa, País Vasco): Ocupaciones Paleolíticas y Postpaleolíticas, vol. B4. Fundación José Miguel de Barandiarán, pp. 149-192.

Altuna, J., 1994. Les macromammifères du Paléolitique supérieur ancien dans la region Cantabrique. In: Bernaldo de Quirós, F. (Ed.), El cuadro cronológico del Paleolítico superior inicial. Museo y centro de investigación de Altamira, monografías $\mathrm{n}^{\circ} 13$, pp. $188-197$.

Altuna, J., Mariezkurrena, K., 1984. Bases de subsistencia de origen animal de los pobladores de Ekain. In: Altuna, J., Merino, J.M. (Eds.), El yacimiento prehistórico de la cueva de Ekain (Deba, Gipuzkoa). Sociedad de Estudios Vascos, San Sebastián, pp. 211-280.

Altuna, J., Mariezkurrena, K., 2010. Tafocenosis en yacimientos del País Vasco con predominio de grandes carnívoros. Consideraciones sobre el yacimiento de Amalda. Zona Arqueol. 13, 215-228.

Altuna, J., Mariezkurrena, K., 2011. Estudio de los macromamíferos del yacimiento de Aitzbitarte III (Excavación de la entrada). In: Altuna, J., Marriezkurrena, K.,
Ríos, J. (Eds.), Ocupaciones humanas en Aitzbitarte III (País Vasco) 33.600-18.400 BP (Zona de entrada de la cueva), vol. 5. EKOB, pp. 395-480.

Altuna, J., Mariezkurrena, K., 2013. Cráneos de pantera en Allekoaitze (Ataun) y Aintzulo (Errezil) (Guipuzkoa). Kobie Ser. Paleoantropol. 32, 29-38.

Álvarez-Laó, D., 2003. Macromamíferos fósiles del Pleistoceno de Asturias. Revisión bibliográfica y datos actuales. Nat. Cantab. 2, 11-23.

Álvarez-Laó, D., 2014. The Jou Puerta cave (Asturias, NW Spain): a MIS 3 large mammal assemblage with mixture of cold and temperate elements. Palaeogeogr. Palaeoclimatol. Palaeoecol. 393, 1-19.

Álvarez-Laó, D., García, N., 2010. Chronological distribution of Pleistocene cold adapted large mammal faunas in the Iberian Peninsula. Quat. Int. 212, 120-128.

Álvarez-Laó, D., García, N., 2011. Geographical distribution of Pleistocene cold adapted large mammal faunas in the Iberian Peninsula. Quat. Int. 233, 159-170.

Antunes, M.T., Cabral, J.M.P., Cardoso, J.L., Pais, J., Soares, A.M., 1989. Paleolítico médio e superior em Portugal: datas 14C, estado actual dos conhecimentos, síntese e discussão. Ciencias Terra (UNL) 10, 127-138.

Anwar, M.B., Jackson, R., Nadeem, M.S., Janečka, J.E., Hussain, S., Beg, M.A., Qayyum, M., 2011. Food habits of the snow leopard Panthera uncia (Schreber, 1775) in Baltistan, Northern Pakistan. Eur. J. Wildl. Res. 57 (5), 1077-1083.

Aparicio, J., Pérez, M. Vives, E., Fumanal, M.P., Dupré, M., 1982. La Cova de les Calaveres (Benidoleig, Alicante). Serie de Trabajos Varios del SIP, vol. 75. Valencia.

Arceredillo, D., 2010. Los macromamíferos del yacimiento del Pleistoceno superior de Prado Vargas (Burgos, España). In: Meléndez, G. (Ed.), III Congreso ibérico de Paleontología. XXVI Jornadas de la Sociedad Española de Paleontología, vol. 9. Publicaciones del Seminario de Paleontología de Zaragoza, pp. 63-66.

Arceredillo, D. Gómez-Olivencia, A. San Pedro-Calleja, Z., 2013. La fauna de micromamíferos de los niveles pleistocenos de la Cueva de Arlanpe (Lemoa, Bizkaia). In: Rios-Garaizar, J., Garate, D., Gómez-Olivencia, A. (Eds.), La Cueva de Arlanpe (Lemoa): ocupaciones humanas desde el Paleolítico medio antiguo hasta la prehistoria reciente. Kobie BAI 3, pp. 123-160.

Argant, A., 2004. Les carnivores du gisement Pliocène final de Saint-Vallier (Drôme, France). Geobios 37, 133-182.

Arribas, A., 1997. Un leopardo, Panthera pardus (Linnaeus, 1758), en el Pleistoceno de la Cueva de los Torrejones (Tamajón, Guadalajara, España). Geogaceta 22, $19-22$.

Arribas, A., Solano, J.G., Garrido, G., Carroza, J.A., 2008. El registro diferencial de carnívoros y homínidos en el karst del Pleistoceno del Sistema Central español: condicionantes geológicos y espaciales. In: Díez, J.C. (Ed.), Zooarqueología Hoy. Encuentros Hispano-Argentinos, pp. 39-52.

Arsuaga, J.L., Villaverde, V., Quam, R., Gracia, A., Lorenzo, C., Martínez, I. Carretero, J.M., 2002. The Gravettian occipital bone from the site of Malladetes (Barx, Valencia, Spain). J. Hum. Evol. 43, 381-393.

Arsuaga, J.L., Baquedano, E., Pérez-González, A., Sala, N., Quam, R.M., Rodríguez, L. García, R., García, N., Álvarez-Lao, D.J., Laplana, C., Huguet, R., Sevilla, P., Maldonado, E., Blain, H.A., Ruiz-Zapata, M.B., Sala, P., Gil-García, M.J., Uzquiano, P., Pantoja, A., Márquez, B., 2012. Understanding the ancient habitats of the last-interglacial (late MIS 5) Neanderthals of central Iberia: paleoenvironmental and taphonomic evidence from the Cueva del Camino (Spain) site. Quat. Int. 275, 55-75.

Ballesio, R., 1980. Le gisement Pléistocène supérieur de la Grotte de Jaurens à Nespouls, Corrèze, France: les carnivores (Mammalia, Carnivora). Lyon Nouv. Arch. Mus. Hist. Nat. 18, 61-102.

Bartosiewicz, L., 2013. Shuffling Nags, Lame Ducks. The Archaeology of Animal Disease Owbow Books.

Baryshnikov, G.F., 2011. Pleistocene Felidae (Mammalia, Carnivora) from the Kudaro cave sites in the Caucasus. Proc. Zool. Inst. RAS 315 (3), 197-226.

Barroso, C., Riquelme, J.A., Moigne, A.M., Banes, L., 2006. Les faunes de grands mammifères du Pléistocène supérieur de la Grotte du Boquete de Zafarraya. Étude Paléontologique, Paléoécologique et Archéozoologique. In: Barroso, C., de Lumley, H. (Eds.), La Grotte du Boquete de Zafarraya, Málaga, pp. 675-891.

Bernaldo de Quirós, F., Maíllo-Fernández, J.M., Castaños, P., Neira, A., 2014. The Gravettian of El Castillo revisited (Cantabria, Spain). Quat. Int. http://dx.doi.org/ 10.1016/j.quaint.2014.07.010.

Bertram, B.C.B., 1999. Leopard. In: Macdonald, D.W. (Ed.), The Encyclopedia of Mammals. Andromeda Oxford, Oxford, pp. 44-48.

Blasco López, R., Rosell, J., Arsuaga, J.L., Bermúdez de Castro, J.M., Carbonell, E., 2010. The hunted hunter: the capture of a lion (Panthera leo fossilis) at the Gran Dolina site, Sierra de Atapuerca, Spain. J. Archaeol. Sci. 37, 2051-2060.

Blasco Sancho, M.F., 1995. Hombres, Fieras y Presas. Estudio arqueozoológico y tafonómico del yacimiento del Paleolítico medio de la Cueva de Gabasa 1 (Huesca). Dpto. Ciencias de la Antigüedad (Área de Prehistoria), Universidad de Zaragoza, Zaragoza.

Bohigas, R., Muñoz, E., 2002. Excavaciones arqueológicas de urgencia en el Covacho de Arenillas (Islares, Castro Urdiales). 1992. In: Actuaciones arqueológicas en Cantabria. Arqueología de gestión. Consejería de cultura, turismo y deporte. Gobierno de Cantabria, Santander, pp. 45-47.

Bonifay, M.F., 1971. Carnivores Quaternaires du Sud-Est de la France. Paris.

Bouchud, J., 1969. La faune moustérienne de Carigüela (Pinar, Grenade). L'Anthropologie 73 (3-4), 361-364.

Boule, M., de Villeneuve, L., 1927. La Grotte de l'Observatoire à Monaco. Paris.

Breuil, H., Obermaier, H., 1914. Travaux en Espagne. L'Anthropologie XXV, 233-253.

Brugal, J.P., Fosse, P., 2004. Carnivores et hommes au quaternaire en Europe de l'Ouest. Rev. Paléobiol. 23 (2), 575-595.

Brugal, J.P., Argant, J., Crispim, J.A., Figueiredo, S., Martín, A., Palmqvist, P., 2012. The 
complex carnivore-rich assemblages from Furninha (Peniche, Portugal): a multidisciplinary approach. J. Taphon. 10 (3-4), 417-438.

Cabrera, V., 1984. El yacimiento de la Cueva de "El Castillo". Biblioteca Praehistorica Hispana, p. 22.

Cáceres, I., Canyelles, J., Esteban, M., Giralt, S., González, F., Huguet, R., Ibáñez, N., Lorenzo, C., Mata, M., Pinto, A., Revilla, A., Rosell, J., Santiago, A., Segura, E., Vallverdú, J., Zaragoza, J., 1993. Estudi d'un exemplar de Panthera pardus i un de Panthera leo spelaea localitzats a l'Abric Romaní (Capellades, Anoia) i anàlisi de la problemática dels carnívors en aquest jaciment. Estrat 6, 31-41.

Camarós, E., Cueto, M., Lorenzo, C., Villaverde, V., Rivals, F., 2015. Large carnivore attacks on hominins during the Pleistocene: a forensic approach with a Neanderthal example. Archaeol. Anthropol. Sci. http://dx.doi.org/10.1007/ s12520-015-0248-1.

Carbonell, E., Estévez, J., Gusi, F., 1979. Resultados preliminares de los trabajos efectuados en el yacimiento del Pleistoceno medio de Cau d'En Borràs (Oropesa, Castellón). Quad. Prehist. Arqueol. Castelló 6, 7-18.

Cardoso, J.L., 1993. Contribução para o conhecimento dos grandes mamíferos do Pleistocénico superior de Portugal. Oeiras.

Cardoso, J.L., 1996. Les grands mammifères du Pléistocène supérieur du Portugal. Essai de synthèse. Geobios 29 (2), 135-250.

Cardoso, J.L., Regala, F.T., 2006. O Leopardo, Panthera pardus (L., 1758), do Algar da Manga Larga (Planalto de Santo António, Porto de Mós). Comun. Geol. 93, 119-144.

Carrión, J.S., Finlayson, C., Fernández, S., Finlayson, G., Allué, E., López-Sanchez, J.A., López-García, P., Gil-Romera, G., Bailey, G., González-Sampériz, P., 2008. A coastal reservoir of biodiversity for Upper Pleistocene human populations: palaeoecological investigations in Gorham's Cave (Gibraltar) in the context of the Iberian Peninsula. Quat. Sci. Rev. 27 (23-24), 2118-2135.

Cassoli, P.F., Tagliacozzo, A., 1991. Considerazioni paleontologiche, paleoecologiche e archeozoologiche sui macromammiferi e gli uccelli dei livelli del Pleistocene Superiore del Riparo di Fumane (VR) (Scavi 1988-1991). Boll. Del Mus. Civ. Storia Nat. Verona 18, 349-446.

Cassoli, P.F., Tagliacozzo, A., 1994. I macromammiferi dei livelli tardopleistoceni delle Arene Candide (Savona, Italia): considerazioni paleontologiche e archeologiche. Quat. Nova 4, 101-262.

Castaños, P.M., 1983. Estudio de los macromamíferos del yacimiento prehistórico de Bolinkoba (Abadiano-Vizcaya). Kobie XIII, 261-298.

Castaños, P.M., 1987. Los carnívoros prehistóricos de Vizcaya. Kobie Ser. Paleoantropol. 16, 7-76.

Castaños, P.M., 2005. Revisión actualizada de las faunas de macromamíferos del Würm antiguo en la Región Cantábrica. Museo de Altamira. Monografías 20, 201-207.

Castaños, P.M., 2010. Nuevas cavidades con carnívoros y humanos en el Cantábrico oriental. Zona Arqueol. 13, 263-268.

Castaños, P.M., Castaños, J., 2007. Estudio de la fauna del Abrigo del Cuco. In: Muñoz, E., Montes, R. (Eds.), Intervenciones arqueológicas en Castro Urdiales 3, pp. 161-170.

Cherin, M., Iurino, D.A., Sardella, R., 2013. Earliest occurrence of Puma pardoides (Owen, 1846) (Carnivora, Felidae) at the Plio/Pleistocene transition in Western Europe: new evidence from the Middle Villafranchian assemblage of Montopoli, Italy. Comptes Rendus Palevol 12 (3), 165-171.

Christiansen, P., 2008. Phylogeny of the great cats (Felidae: Pantherinae), and the influence of fossil taxa and missing characters. Cladistics 24, 977-992.

Christiansen, P., Harris, J.M., 2012. Variation in craniomandibular morphology and sexual dimorphism in pantherines and the sabercat smilodon fatalis. Plos One 7 (10), e48352.

Corchón, S., 2014. La Cueva de las Caldas (Priorio, Norte de España). In: Sala, R., Carbonell, E., Bermúdez de Castro, J.M., Arsuaga, J.L. (Eds.), Los cazadores recolectores del Pleistoceno y del Holoceno en Iberia y el Estrecho de Gibraltar. Estado actual del conocimiento del registro arqueológico. Universidad de Burgos y Fundación Atapuerca, pp. 64-75.

Corral, J.C., 2012. Técnicas aplicadas en la preparación de un cráneo cuaternario de Panthera pardus (Linneo, 1758) de Ataun (cueva de Allekoaitze, Guipúzcoa, España). Bol. Geol. Min. 123 (2), 127-138.

Crégut-Bonnoure, E., 1996. Ordre des Carnivores. In: Guérin, C., Patou-Mathis, M. (Eds.), Les grands Mammifères Plio-Pléistocènes d'Europe. Paris, pp. 155-230.

Currant, A.P., 2000. A review of the quaternary mammals of Gibraltar. In: Stringer, C.B., Barton, R.N.E., Finlayson, J.C. (Eds.), Neanderthals on the Edge. Oxbow books, pp. 201-205.

Dari, A., 1999. Les grands mammifères du site Pléistocène supérieur de la grotte du Castillo. Étude archéozoologique: donnes paléontologiques, taphonomiques et palethnographiques. Espacio, Tiempo y Forma, Serie I. Prehist. Arqueol. 12, 103-127.

Daura, J., Sanz, M., Subirà, M.E., Quam, R., Fullola, J.M., Arsuaga, J.L., 2005. A neanderthal mandible from the Cova del Gegant (Sitges, Barcelona, Spain). J. Hum. Evol. 49 (1), 56-70.

Daura, J., Sanz, M., García, N., Allué, E., Vaquero, M., Fierro, E., Carrión, J.S., LópezGarcía, J.M., Blain, H.A., Sánchez-Marco, A., Valls, C., Albert, R.M., Fornós, J.J., Julià, R., Fullola, J.M., Zilhão, J., 2013. Terrasses de la Riera dels Canyars (Gavà, Barcelona): the landscape of Heinrich Stadial 4 north of the "Ebro frontier" and implications for modern human dispersal into Iberia. Quat. Sci. Rev. 60, 26-48.

Davidson, I., 1989. La economía del final del Paleolítico en la España oriental. In: Servicio de Investigación Prehistórica de la Diputación de Valencia, Serie de Trabajos Varios del SIP 85, Valencia.

Davis, S.J.M., 2002. The mammals and birds from the Gruta do Caldeirão, Portugal.
Rev. Port. Arqueol. 5 (2), 29-98.

Delibes, M., 1972. Informe paleontológico de la fauna de la Cueva de la Ermita. In: Moure, J.A., Delibes, G.A. (Eds.), El yacimiento musteriense de la Cueva de la Ermita. Noticiario Arqueológico Hispánico. Prehistoria 1, pp. 41-44.

De Lombera-Hermida, A., Rodríguez, X.P., Fábregas-Valcarce, R., 2014. El yacimiento arqueo-paleontológico de Cova Eirós (Triacastela, Lugo). In: Sala, R. (Ed.), Los cazadores recolectores del Pleistoceno y Holoceno en Iberia y el Estrecho de Gibraltar: Estado actual del conocimiento del registro arqueológico. Universidad de Burgos y Fundación Atapuerca, pp. 18-25.

De la Peña, P., 2013. Estudio estratigráfico y tecnotipológico de los niveles basales de la Cueva de les Mallaetes (Barx, Valencia): nuevas claves para el Paleolítico superior inicial mediterráneo. Zephyrus LXXI (1), 61-88.

De la Rasilla, M., Santamaría, D., 2011-2012. El Paleolítico medio en Asturias. Mainake XXXIII, 31-62.

de Ruiter, D.J., Berger, L.R., 2000. Leopards as taphonomic agents in dolomitic cavesimplications for bone accumulations in the hominid-bearing deposits of South Africa. J. Archaeol. Sci. 27, 665-684.

Diedrich, C.G., 2007. Upper Pleistocene Panthera leo spelaea (Goldfuss, 1810) skeleton remains from Praha-Podbabaand other lion finds from loess and river terrace sites in Central Bohemia (Czech Republic). Bull. Geosci. 82 (2), 99-117.

Diedrich, C.G., 2009. Steppe lion remains imported by ice age spotted hyenas into the Late Pleistocene Perick Caves hyena den in Northern Germany. Quat. Res. 71 (3), $361-374$

Diedrich, C.G., 2011. A clan of Late Pleistocene hyenas, Crocuta Crocuta spelaed (Goldfuss, 1823), from the Rösenbeck Cave (Germany) and a contribution to cranial shape variability. Biol. J. Linn. Soc. 103 (1), 191-220.

Diedrich, C.G., 2013. Late Pleistocene leopards across Europe-northernmost European German population, highest elevated records in the Swiss Alps, complete skeletons in the Bosnia Herzegowina Dinarids and comparison to the ice age cave art. Quat. Sci. Rev. 76, 167-193.

Diedrich, C.G., 2014. Palaeopopulations of Late Pleistocene top predators in Europe: Ice age spotted hyenas and steppe lions in battle and competition about prey. Paleontol. J. http://dx.doi.org/10.1155/2014/106203.

Diedrich, C.G., Žák, K., 2006. Prey deposits and den sites of the Upper Pleistocene hyena Crocuta crocuta spelaea (Goldfuss, 1823) in horizontal and vertical caves of the Bohemian karst (Czech Republic). Bull. Geosci. 4 (81), 237-276.

Díez, J.C., García, M.A., Gil, E., Jordá, J.F., Ortega, A.I., Sánchez, A., Sánchez, B., 1988-89. La cueva de Valdegoba (Burgos). Primera campaña de excavaciones. Zephyrus XLI-XLII, 55-74.

Domínguez-Rodrigo, M., Pickering, M.T., 2010. A multivariate approach for discriminating bone accumulations created by spotted hyenas and leopards: harnessing actualistic data from east and Southern Africa. J. Taphon. 8 (2-3), 155-179.

Driesch, A., von, D., 1976. A Guide to the Measurement of Animal Bones from Archaeological Sites. Peabody Museum Bulletin $N^{\circ} 1$. Harvard University.

Estévez, J., 1975-76. Hallazgo de una pantera en el Pleistoceno catalán. Speleon 22, $171-178$.

Estévez, J., 1979. Tesis doctoral. La fauna del Pleistoceno catalán, vol. 2. Universitat de Barcelona.

Estévez, J., 1987. La fauna de L'Arbreda (s. alfa) en el conjunt de faunes del Pleistocè superior. Cypsela VI, 73-87.

Falguères, C., Yokohama, Y., Arrizabalaga, A., 2005-2006. La geocronología del yacimiento pleistocénico de Lezetxiki (Arrasate, País Vasco). Crítica de las dataciones existentes y algunas nuevas aportaciones. Munibe 57 (2), 93-106.

Fernández, J., 2004. Datos sobre la incidencia de carnívoros en la Cova del Bolomor (Tavernes de la Valldigna, Valencia). In: Miscelánea en homenaje a Emiliano Aguirre, vol. IV. Museo Arqueológico Regional, pp. 141-157.

Fernández, J., 2007. La Cova del Bolomor (Tavernes de la Valldigna, Valencia). In: Las industrias líticas del Pleistoceno medio en el ámbito del Mediterráneo peninsular. Serie de Trabajos Varios del SIP 108, Valencia.

Fischer, K., 2000. Ein Leoparden-Fund, Panthera pardus (L., 1758) aus dem jungpleistozänen Rixdofer Horizont von Berlin und die Verbritung dei Leoparden im Pleistozän Europas. Mitt. Mus. Nat.kd. Berl. Geowiss. Reihe 3, 221-227.

Fortea, J., Jordá, F., 1976. La Cueva de les Mallaetes y los problemas del Paleolítico superior del Mediterráneo español. Zephyrus XXVI-XXVII, 129-166.

Fortea, J., De la Rasilla, M., Rodríguez, V., 1992. La cueva de Llonín (Llonín, Peñamera Alta). Campañas de 1987 a 1990. In: Excavaciones arqueológicas en Asturias 1987-1990. N N $^{\circ}$, pp. 9-18.

Fortea, J., De la Rasilla, M., Rodríguez, V., 1995. La cueva de Llonín (Llonín, Peñamera Alta). Campañas de 1991 a 1994. In: Excavaciones arqueológicas en Asturias 1991-1994. N N $^{\circ}$, pp. 33-43.

Fortea, J., De la Rasilla, M., Rodríguez, V., 1999. La cueva de Llonín (Llonín, Peñamera Alta). Campañas de 1995 a 1998. In: Excavaciones arqueológicas en Asturias 1995-1998. $\mathrm{N}^{\circ} 4$, pp. 59-73.

Fraga, E., 1958. Catálogo bibliográfico de la fauna cuaternaria asturiana. Monografías Geológicas VIII. Instituto de Geología Aplicada, Oviedo.

Gabucio, M.J., Cáceres, I., Rodríguez-Hidalgo, A., Rosell, J., Saladié, P., 2014. A wild cat (Felis silvestris) butchered by Neanderthals in Level O of the Abric Romaní site (Capellades, Barcelona, Spain). Quat. Int. 326-327, 307-318.

Galván, B., Hernández, C.M., Mallol, C., Mercier, N., Sistiaga, A., Soler, V., 2014. New evidence of early Neanderthal disappearance in the Iberian Peninsula. J. Hum. Evol. 75, 16-27.

García, N., 2003. Osos y Otros Carnívoros de la Sierra de Atapuerca. Fundación Oso de Asturias, Oviedo.

Ghezzo, E., Rook, L., 2015. The remarkable Panthera pardus (Felidae, Mammalia) 
record from Equi (Massa, Italy): taphonomy, morphology, and paleoecology. Quat. Sci. Rev. 110, 131-151.

Gidna, A., Domínguez-Rodrigo, M., Pickering, T.R., 2015. Patterns of bovid long limb bone modification created by wild and captive leopards and their relevance to the elaboration of referential frameworks for paleoanthropology. J. Archaeol. Sci. Rep. (2015). http://dx.doi.org/10.1016/j.jasrep.2015.03.003.

Hart, J.A., Katembo, M., Punga, K., 1996. Diet, prey selection and ecological relations of leopard and golden cat in the Ituri Forest, Zaire. Afr. J. Ecol. 34, 364-379.

Hayward, M.W., Henschel, P., O'Brien, J., Hofmeyr, M., Balme, G., Kerley, G.I.H., 2006. Prey preferences of the leopard (Panthera pardus). J. Zool. 270 (2), 298-313.

Hemmer, H., 1971. Zur Kenntnis pleistozäner mitteleuropaïscher Leoparden (Panthera pardus). Neues Jahrbuch für Geologie und Paläontologie. Abhandlungen 138 (1), 15-36.

Hemmer, H., 1972. Uncia uncia. Mamm. Species 20, 1-5.

Hemmer, H., 2001. Die Feliden aus den Epivillafranchium von Untermaßfeld. In: Kahlke, R.D. (Ed.), Das Pleistozän von Untermaßfeld bei Meimingen (Thüringen), Tell 3, Monographien des Römisch-Gemanischen Zentralmuseums, Bd 40, 3, pp. 699-1030. I-VI.

Hemmer, H., Kahlke, R.D., Vekua, A., 2004. The old World puma-Puma pardoides (Owen, 1846) (Carnivora: Felidae)-in the lower Villafranchian (Upper Pliocene) of Kvabebi (East Georgia, Transcaucasia) and its evolutionary and biogeographical significance. Neues Jahrb. Geol. Palaeontol. Abh. 233, 197-231.

Indu, V.R., Sreeranjini, A.R., Rajani, C.V., Ashok, N., 2013. Gross observations on the cervical vertebrae of leopard (Panthera pardus). J. Vet. Anim. Sci. 44, 63-65.

Iñigo, C., Molero, G., Maldonado, E., 1998. Los carnívoros del yacimiento pleistoceno de Cueva del Búho (Segovia, España) y sus huellas de actividad. Estud. Geol. 54 65-73.

Juan-Cabanilles, J., 1991. La Cova del Racó del Duc. In: Ferrairó, J.M., Mascarell, J.V., Romaguera, F., Sastre, F., Vilaplana, J. (Eds.), El Racó del Duc a peu, un itinerar de la natura. GEMAS (Grup d'Estudis Medio-Ambientals de la Safor). Conselleria d'Administració Pública, Agència de Medi Ambient, Generalitat Valenciana, pp. 266-267. Monografies 11.

Juan-Cabanilles, J., 1997. La Cova del Racó del Duc i els primers pobladors de Vilallonga. Bresca 0 (2 época), pp. 12-14.

Kingdon, J., 1997. The Kingdon Field Guide to African Mammasls. Academic Press, New York.

Klein, R., Cruz-Uribe, K., 1985. La fauna mamífera del yacimiento de la cueva de E Juyo. Campañas de 1978 y 1979. In: Barandiarán, I., Freeman, L.J., González, J., Klein, R. (Eds.), Excavaciones en la cueva de El Juyo. Monografías, vol. 14. Centro de Investigación y Museo de Altamira, pp. 99-120.

Kotsakis, T., Palombo, M.R., 1979. Un cranio di Panthera pardus (L.) del Pleistocene medio superiore di Monte Sacro (Roma). Geol. Romana 18, 137-155.

Kruuk, H., 2002. Hunter and Hunted. Relationships between Carnivores and People. Cambridge University Press.

Lyman, R.L., 2008. Quantitative Paleozoology. Cambridge Manuals in Archaeology. Cambridge University Press.

Macdonald, D.W., Loveridge, A.J., Nowell, K., 2010. Dramatis personae: an introduction to the wild felids. In: Macdonald, D.W., Loveridge, A. (Eds.), The Biology and Conservation of Wild Felids. Oxford University Press, pp. 1-58.

Madurell-Malapeira, J., Alba, D.M., Moyà-Solà, S., Aurell-Garrido, J., 2010. The Iberian record of the puma-like cat Puma pardoides (Owen, 1846) (Carnivora, Felidae). Comptes Rendus Palevol 9 (1-2), 55-62.

Marciszak, A., Krajcarz, M.T., Krajcarz, M., Stefaniak, K., 2011. The first record of leopard Panthera pardus LINNAEUS, 1758 from the Pleistocene of Poland. Acta Zool. Cracov. 54A (1-2), 39-46.

Mariezkurrena, K., 1987. Macromamíferos asociados a los enterramientos calcolíticos de la cueva Iruaxpe I (Guipúzcoa). Munibe 39, 80-84.

Mariezkurrena, K., 2011. Hallazgos de macromamíferos poco frecuentes en yacimientos arqueológicos y paleontológicos del Pleistoceno de la Región Cantábrica. Kobie Ser. Paleoantropol. 30, 83-110.

Marín-Arroyo, A.B., 2009. A comparative study of analytic techniques for skeletal part profile interpretation at El Mirón Cave (Cantabria, Spain). Archaeofauna 18 79-98.

Maroto, J., Soler, N., Mir, A., 1987. La cueva de Mollet I (Serinyà, Girona). Cypsela 6 , $101-110$.

Maroto, J., 1993. La cueva de los Ermitons (Sales de Llierca, Girona): un yacimiento del Paleolítico medio final. Espacio, Tiempo y Forma. Serie I. Prehist. Arqueol. 6, $13-30$.

Maroto, J., Julià, R., López-García, J.M., Blain, H.A., 2012. Chronological and environmental context of the Middle Pleistocene human tooth from Mollet cave (Serinyà, NE Iberian Peninsula). J. Hum. Evol. 62, 655-663.

Martínez, R., 1996. Fauna del Pleistoceno superior en el País Valenciano: aspectos económicos, huella de manipulación y valoración paleoambiental. Tesis doctoral inédita. Universitat de València.

Martínez, R., 2009. Restos óseos de macromamíferos y aves. In: Villaverde, V. Pérez, J., Lledó, J.C. (Eds.), Los primeros pobladores de La Costera: los neandertales de la Cova Negra de Xàtiva. Historia de Xàtiva. Prehistoria, Arqueología y Antigüedad, vol. I. Universitat de València, Xàtiva, pp. 59-83.

Mazo, C., Utrilla, P., Blasco, F., Mandado, J., Torres, T., Ortiz, E., Rink, W.J., 2011-2012 El nivel musteriense de la Cueva de Abauntz (Arraitz, Navarra) y su aportación al debate "Vasconiense". Mainake XXXIII, 187-214.

Menéndez, M., Weniger, G-Ch, Álvarez-Alonso, D., De Andrés-Herrero, M., García, E. Jordá, J.F., Kehl, M., Rojo, J., Quesada, J.M., Schmidh, I., 2014. La Cueva de la Güelga. Cangas de Onís. Asturias. In: Sala, R., Carbonell, E., Bermúdez de Castro, J.M., Arsuaga, J.L. (Eds.), Los cazadores recolectores del Pleistoceno y del
Holoceno en Iberia y el Estrecho de Gibraltar. Estado actual del conocimiento del registro arqueológico. Universidad de Burgos y Fundación Atapuerca, pp. 60-63.

Mills, M.G.L., Harvey, M., 2001. African Predators. Struik Publishers, Cape Town.

Molero, G., Maldonado, E., Íñigo, C., Sánchez, F.L., Díez, A., 1989. El yacimiento del Pleistoceno superior de la Cueva del Búho (Perogordo, Segovia) y su fauna de vertebrados. In: V Jornadas de Paleontología. Universitat de València, València, pp. 101-102.

Morales, J.V., Sanchis, A., Real, C., Pérez, M., Aura, J.E., Villaverde, V., 2012. Evidences of interaction Homo-Cuon in three Upper Pleistocene sites of the Iberian Mediterranean central region. J. Taphon. 10 (3-4), 463-476.

Morris, P., 1972. A review of mammalian age determination methods. Mamm. Rev. 2, 69-104.

Moullé, P.E., Lacombat, F., Echassoux, A., 2006. Apport des grands mammifères de la grotte du Vallonnet (Roquebrune-Cap-Martin, Alpes-Maritimes, France) à la connaissance du cadre biochronologique de la seconde moitié du Pléistocène inférieur d'Europe. L'Anthropologie 110 (5), 837-849.

Nadal, J., de Haro, S., Maroto, J., 2002. Els grans mamífers del Plistocè superior. Quad. (CECB) 23, 155-180.

Nagel, D., 1999. Panthera pardus vraonensis n. ssp., a new leopard from the Pleistocene of Vraona/Greece. N. Jb. Geol. Palaont. Mh. 1999 (3), 129-150.

Nowel, K., Jackson, P., 1996. Wild Cats: Status Survey and Conservation Action Plan. IUCN (World Conservation Union), Gland.

Oli, M.K., Taylor, I.R., Rogers, M.E., 1993. Diet of the snow leopard (Panthera uncia) in the Annapurna conservation Area, Nepal. J. Zool. 231, 365-370.

O'Regan, H.J., 2008. The Iberian Peninsula-corridor or cul-de-sac? Mammalian faunal change and possible routes of dispersal in the last 2 million years. Quat. Sci. Rev. 27, 2136-2144.

O'Regan, H.J., Turner, A., 2004. Biostratigraphic and paleoecological implications of the new fossil felid material from the Plio-Pleistocene site of Tegelen, the Netherlands. Palaeontology 47 (5), 1181-1193.

Pantoja, A., Sala, M.T.N., García, N., Ruiz, B., Gil, M.J., Aranburu, A., Arsuaga, J.L., Casabó, J., 2011. Análisis paleontológico del yacimiento del Pleistoceno superior de Cova Foradada (Xàbia, Alicante, España). Bol. R. Soc. Esp. Hist. Nat. Sec. Geol. 105 (1-4), 53-66.

Pérez, M., 1977. Los mamíferos del yacimiento musteriense de Cova Negra. In: Servicio de Investigación Prehistórica de la Diputación de Valencia, Serie de Trabajos Varios del SIP 53, Valencia.

Pérez, M., Morales, J.V., Sanchis, A., Aura, J.E., Sarrión, I., 2010. Presence of the genus Cuon in upper Pleistocene and initial Holocene sites of the Iberian Peninsula: new remains identified in archaeological contexts of the Mediterranean region. J. Archaeol. Sci. 37, 437-450.

Podhade, D.N., Shrivastav, A.B., Vaish, R., 2013. Osteomorphometrical study of femur of the leopard (Panthera pardus). J. Wildl. Res. 1 (1), 1-4.

Podhade, D.N., Shrivastav, A.B., Vaish, R., 2014a. Gross osteomorphometrical study of ossa coxarum of the leopard (Panthera pardus). Veterinary World 7, 10-12.

Podhade, D.N., Shrivastav, A.B., Vaish, R., Tiwari, Y., 2014b. Morphology and morphometry of tarsals of the leopard (Panthera pardus). Res. J. Anim. Vet. Fish. Sci. 2 (12), 20-21.

Pons-Moyà, J., 1987. Los carnívoros (Mammalia) de Venta Micena (Granada, España). Paleontologia i Evolució. Mem. Espec. 1, 109-128.

Quesada, J.M., 2006. Faunas del Auriñaciense y Gravetiense cantábrico: Asturias y Santander. Revisión crítica y periodización. Zona Arqueol. 7 (1), 406-421.

Quam, R.M., Arsuaga, J.L., Bermúdez de Castro, J.M., Díez, J.C., Lorenzo, C., Carretero, J.M., García, N., Ortega, A.I., 2001. Human remains from Valdegoba cave (Huérmeces, Burgos, Spain). J. Hum. Evol. 41, 385-435.

Rasines del Río, P., Muñoz, E., Santamaría, S., Morlote, J.M., Gutiérrez, I., 2011. Aproximación paleoambiental al nivel XIII (Auriñaciense) del Abrigo del Cuco (Castro Urdiales. Cantabria). Kobie Ser. Paleoantropol. 30, 31-42.

Rivas-Martínez, S., 1987. Memoria del mapa de series de vegetación de España 1: 400.000. I.C.O.N.A, Madrid.

Rivas-Martínez, S., Díaz, T.E., Fernández, F., Izco, J., Loidi, J., Lousa, M., Peñas, A., 2002. Vascular plant communities of Spain and Portugal. Itinera Geobot. 15.

Rosado-Méndez, N.Y., Lloveras, L., Daura, J., Nadal, J., Sanz, M., 2014. Predator agents and leporid accumulations: the case of Terrasses de la Riera dels Canyars (Gavà, Barcelona, Spain). J. Archaeol. Method Theory. http://dx.doi.org/10.1007/ s10816-014-9214-y.

Rodríguez, J., Giles, F., Riquelme, J.A., 2010. El registro fósil de hienas en las cuevas de Gorham's y Vanguard (Gibraltar): contexto paleogeográfico. Zona Arqueol. 13, 354-365.

Royo, J., 1942. Cova Negra de Bellús. II. Relación detallada del material fósil. In: Serie de Trabajos Varios del SIP 6, Valencia, pp. 14-18.

Sala, M.T.N., Arsuaga, J.L., Laplana, C., Ruiz, B., Gil, M.J., García, N., Aranburu, A., Algaba, M., 2011. Un paisaje de la Meseta durante el Pleistoceno Superior. Aspectos paleontológicos de la Cueva de la Zarzamora (Segovia, España). Bol. R. Soc. Esp. Hist. Nat. Sec. Geol. 105 (1-4), 67-85.

Sala, M.T.N., Algaba, M., Arsuaga, J.L., Aranburu, A., Pantoja, A., 2012. A taphonomic study of the Búho and Zarzamora caves. Hyenas and humans in the Iberian plateau (Segovia, Spain) during the Late Pleistocene. J. Taphon. 10 (3-4), 477-497.

Sarrión, I., 1976. Apuntes sobre fauna cuaternaria. Lapiaz 3, 15-22.

Sarrión, I., 1984. La fauna del Würm superior de la Cueva Merino (Dos Aguas, Valencia). Lapiaz 13, 31-36.

Sarrión, I., 1990. El yacimiento del Pleistoceno medio de la Cova del Corb (Ondara, Alicante). Arch. Prehist. Levant. XX, 43-77. 
Sauqué, V., Cuenca-Bescós, G., 2013. The Iberian Peninsula, the last European refugium of Panthera pardus LINNAEUS 1758 during the Upper Pleistocene. Quaternaire 24 (1), 35-48.

Sauqué, V., Rabal-Garcés, R., Cuenca-Bescós, G., 2014a. Carnivores from Los Rincones, a leopard den in the highest mountain of the Iberian range (Moncayo, Zaragoza, Spain). Hist. Biol. http://dx.doi.org/10.1080/0891263.2014.977882.

Sauqué, V., Rabal-Garcés, R., Sola-Almagro, C., Cuenca-Bescós, G., 2014b. Bone accumulation by leopards in the late Pleistocene in the Moncayo Massif (Zaragoza, NE Spain). Plos One. http://dx.doi.org/10.1371/journal.pone.0092144.

Sauqué, V., García-González, R., Cuenca-Bescós, G., 2014c. A Late Pleistocene (MIS 3) ungulate mammal assemblage (Los Rincones, Zaragoza, Spain) in the Eurosiberian-Mediterranean boundary. Hist. Biol. http://dx.doi.org/10.1080/ 0891263.2014 .945926$.

Sauqué, V., Galán, J., Núñez-Lahuerta, C., Sola-Almagro, C., Rabal-Garcés, R., Martínez, I., Maxo, C., Cuenca-Bescós, G., 2014d. Neandertales en el Pleistoceno superior del Valle del Ebro, paleoambiente y evidencias tafonómicas en Aguilón P-7. Fundamental 223-226.

Sims, M.E., 2005. Identification of Mid-size Cat Skulls. Identification Guides for Wildlife Law Enforcement 7. USFWS. National Fish and Wildlife Forensics Laboratory, Ashland, OR.

Sims, M.E., 2012. Cranial morphology of five felids: Acinonyx jubatus, Panthera onca, Panthera pardus, Puma concolor, Uncia uncia. Russ. J. Theriol. 11 (2), 157-170.

Schmid, E., 1940. Variationsstatistische Untersuchungen am Gebiß pleitozäner und rezenter Leoparden und anderer Feliden. Z. für Säugetierkd. 15 (1), 1-179.

Sommer, R.S., Benecke, N., 2006. Late Pleistocene and Holocene development of the felid fauna (Felidae) of Europe: a review. J. Zool. 269, 7-19.

Spassov, N., Raychev, D., 1997. Late Würm Panthera pardus remains from Bulgaria: the European fossil leopards and the question of the probable species survival until the Holocene on the Balkans. Hist. Nat. Bulg. 7, 71-96.

Stander, P.E., 1997. Field age determination of leopards by tooth wear. Afr. J. Ecol. 35, $156-161$.

Stein, A.B., Hayssen, V., 2013. Panthera pardus (Carnivora: Felidae). Mamm. Species $47(1), 30-48$

Stringer, C., Finlayson, J.C., Barton, R.N.E., Cáceres, I., Fernández-Jalvo, Y., 2008 Neanderthal exploitation of marine mammals in Gibraltar. PNAS 105 (38), $14319-14324$.

Testu, A., 2006. Étude paléontologique et biostratigraphique des Felidae et Hyaenidae pléistocènes de l'Europe méditerranéenne. Thèse de doctorat. Université de Perpignan, Perpignan.

Testu, A., Moigne, A.M., de Lumley, H., 2011. La panthère Panthera pardus des niveux inférieurs de la Caune de L'Arago à Tautavel (Pyrénées-Orientales, France) dans le contexte des Felidae (Felinae, Pantherinae) de taille moyenne du Pléistocène européen. Quat. Hors-série (4), 271-281.

Turner, A., 1995. Variaciones regionales en la fauna de grandes mamíferos del Pleistoceno inferior y medio de Europa. Una perspectiva ibérica. In: Evolución humana en Europa y los yacimientos de la Sierra de Atapuerca, pp. 57-73.

Turner, A., Antón, M., 1997. The Big Cats and Their Fossil Relatives. Columbia University Press, New York.

Turner, A., O'Regan, H.J., 2002. The assessment of size in fossil Felidae. Estud. Geol. $58,45-54$.

Utrilla, P., Montes, L., Blasco, F., Torres, T., Ortega, J.E., 2010. La cueva de Gabasa revisada 15 años después: un cubil para las hienas y un cazadero para los neandertales. Zona Arqueol. 13, 376-391.

Utrilla, P., Mazo, C., Domingo, R., 2014. Fifty thousand years of prehistory at the cave of Abauntz (Arraitz, Navarre): a nexus point between the Ebro Valley, Aquitaine and the Cantabrian corridor. Quat. Int. http://dx.doi.org/10.1016/ j.quaint.2014.06.022.
Valente, M.J., 2004a. Humanos e carnívoros no Paleolítico Superior inicial em Portugal: arqueozoologia e tafonomia da gruta do Pego do Diabo (Loures). Promontoria 2, 107-142.

Valente, M.J., 2004b. Humans and carnivores in the early upper Paleolithic in Portugal: data from Pego do Diabo cave. Rev. Paléobiol. 23 (2), 611-626.

Vega del Sella, C., 1930. Las cuevas de la Riera y Balmori (Asturias). Mem. Com. Investig. Paleontol. Prehist. 13.

Villaluenga, A., 2009. Yacimientos del Pleistoceno Superior en la Península Ibérica con presencia de restos de oso. Munibe 60, 17-33.

Villaluenga, A., Castaños, P., Arrizabalaga, A., Mujika, J.A., 2012a. Cave Bear (Ursus spelaeus Rosenmüller Heinroth, 1794) and humans during the early Upper Pleistocene (Lower and Middle Palaeolithic) in Lezetxiki, Lezetxiki II and Astigarragako Kobea (Basque country, Spain). Preliminary approach. J. Taphon. $10(3-4), 521-543$.

Villaluenga, A., Arrizabalaga, A., Rios-Garaizar, J., 2012b. Multidisciplinary approach to two Chatelperronian series: lower IX layer of Labeko Koba and X Level of Ekain (Basque country, Spain). J. Taphon. 10 (3-4), 499-520.

Villaverde, V., 2009. Los primeros pobladores de La Costera: los neandertales de la Cova Negra de Xàtiva. In: Villaverde, V., Pérez, J., Lledó, J.C. (Eds.), Los primeros pobladores de La Costera: los neandertales de la Cova Negra de Xàtiva. Historia de Xàtiva. Prehistoria, Arqueología y Antigüedad, vol. I. Universitat de València, Xàtiva.

Villaverde, V., Guillem, P., Martínez, R., Eixea, A., 2014. Cova Negra. In: Carbonell, E. Bermúdez de Castro, J.M., Arsuaga, J.L. (Eds.), Los cazadores recolectores del Pleistoceno y del Holoceno en Iberia y el Estrecho de Gibraltar. Fundación Atapuerca. Universidad de Burgos, pp. 361-369.

Viñas, R., Villalta, J.F., 1975. El depósito cuaternario de la “Cova del Gegant”. Speleon, Monografía I, pp. 19-33.

Walker, M.J., 2001. Excavations at Cueva Negra del Estrecho del Rio Quipar and Sima de las Palomas del Cabezo Gordo: two sites in Murcia (south-east Spain) with Neanderthal skeletal remains, Mousterian assemblages and late Middle to early Upper Pleistocene fauna. In: Milliken, S., Cook, J. (Eds.), A Very Remote Period Indeed: Papers on the Palaeolithic Presented to Derek Roe. Oxbow Books, Oxford.

Walker, M.J., López-Martínez, M.V., Ortega-Rodrigáñez, J., Haber-Uriarte, M., LópezJiménez, A., Avilés-Fernández, A., Polo-Camacho, J.L., Campillo-Boj, M., GarcíaTorres, J., Carrión-García, J.S., San Nicolás-del Toro, M., Rodríguez-Estrella, T. 2012. The excavation of buried articulated Neanderthal skeletons at Sima de las Palomas (Murcia, SE Spain). Quat. Int. 259, 7-21.

Wolsan, M., 1993. Évolution des carnivores quaternaires en Europe central dans leur contexte stratigraphique et paléoclimatique. L'Anthropologie 97 (2/3), 203-222.

Wood, R.E., Barroso, C., Caparrós, M., Jordá, J.F., Galván, B., Higham, T., 2013. Radiocarbon dating casts doubt on the late chronology of the Middle to Upper Paleolithic transition in southern Iberia. PNAS 110 (8), 2781-2786.

Yravedra, J., 2005. Aprovechamiento cárnico de lince (Lynx pardina) durante el Pleistoceno superior en el interior de la Península Ibérica. Munibe 57, 303-313.

Yravedra, J., 2010a. A taphonomic perspective on the origins of the faunal remains from Amalda Cave (Spain). J. Taphon. 8 (4), 301-334.

Yravedra, J., 2010b. Zooarqueología y tafonomía del yacimiento de Hornos de la Peña (San Felices de Buelna, Cantabria). Complutum 21 (1), 69-86.

Zilhão, J., Davis, S.J.M., Duarte, C., Soares, A.M.M., Steier, P., Wild, E., 2010a. Pego do Diabo (Loures, Portugal): dating the Emergence of anatomical Modernity in Westernmost Eurasia. Plos One 5 (1), 1-22.

Zilhão, J., Angelucci, D.E., Argant, J., Brugal, J.P., Carrión, J.S., Carvalho, R., Fuentes, N. Nabais, M., 2010b. Humans and hyenas in the Middle Paleolithic of Gruta da Oliveira (Almonda System, Torres Novas, Portugal). Zona Arqueol. 13, 298-309. 